\title{
C-H Halogenation of Pyridyl Sulfides Avoiding the Sulfur Oxidation: a Direct Catalytic Access to Sulfanyl Polyhalides and Polyaromatics
}

\author{
Johan Guilbaud, ${ }^{\dagger}$ Awatef Selmi, ${ }^{\dagger}, \ddagger$ Majed Kammoun, ${ }^{\ddagger}$ Sylvie Contal, ${ }^{\S}$ Christian \\ Montalbetti, ${ }^{\S}$ Nadine Pirio, ${ }^{\dagger}$ Julien Roger, ${ }^{*}{ }^{\dagger}$ and Jean-Cyrille Hierso ${ }^{*}+\Pi$
${ }^{\dagger}$ Institut de Chimie Moléculaire de l'Université de Bourgogne (ICMuB), UMR-CNRS 6302, Université de Bourgogne Franche-Comté (UBFC), 9 avenue Alain Savary, 21078 Dijon, France
₹ Institut Supérieur de Biotechnologie, Unité de Recherche de Chimie Médicinale et Environnementale (UR-17-ES-40), Université de Sfax, route Soukra Km 4 BP1175-3038, Sfax, Tunisie
§ Inventiva, 50 rue de Dijon 21121 Daix (France)
${ }^{\Pi}$ Institut Universitaire de France (IUF), 103 Boulevard. Saint Michel, 75005 Paris Cedex, France

\section{$\underline{\text { Table of contents }}$}

General conditions.

Scheme S1: Molecules naming and numbering......

$\mathrm{C}-\mathrm{H}$ bromination reaction in undried chlorobenzene

Kinetic measurements of the $\mathrm{C}-\mathrm{H}$ bromination of chlorophenyl sulfanyl pyridine (1).....

Detailed work-up procedure S6

Fluorination reaction of (14). S7

Figures S5.1-S5.39: ${ }^{1} \mathrm{H},{ }^{13} \mathrm{C}$ and ${ }^{19} \mathrm{~F}$ NMR copy of new products. S9 
Supporting information section (including figures and tables) is also reported in the archived thesis: Johan Guilbaud - 06-12-2018 (in French). Synthèses d'(arylsulfanyl)pyridines halogénées et leurs dérivés par couplages $C-S$ et $C-X$ catalysés par des complexes du palladium. Catalyse. Université Bourgogne Franche-Comté, 2018. Français. NNT: 2018UBFCK047. tel-02067489). ${ }^{1}$

\section{General conditions}

Reagents were purchased from commercial suppliers and used without purification. Unless otherwise stated, the solvent used were anhydrous: Specifically, chlorobenzene anhydrous 99.8\% (Sigma-Aldrich Sure/Seal, $<0.005 \%$ water) and 1,4-dioxane extra dry $99.8 \%$ (Acros Organics AcroSeal, $<0.005 \%$ water). Reactions were performed under an argon atmosphere using vacuum-lines and Schlenk techniques. ${ }^{1} \mathrm{H}(500 \mathrm{MHz}),{ }^{13} \mathrm{C}(126 \mathrm{MHz}),{ }^{19} \mathrm{~F}(470 \mathrm{MHz})$ NMR were recorded on Bruker AVANCE III instruments in $\mathrm{CDCl}_{3}$ solutions. Chemical shifts are reported in ppm relative to $\mathrm{CDCl}_{3}\left({ }^{1} \mathrm{H}: 7.26\right.$ and ${ }^{13} \mathrm{C}$ : 77.16), and coupling constants $J$ are given in $\mathrm{Hz}$. GC-MS experiments were performed with a Trace GC Ultra equipped with a mass-selective detector, and high resolution mass spectra (HRMS) were obtained on a Thermo LTQ-Orbitrap XL with ESI source. Flash chromatography was performed on silica gel (230400 mesh) or with PuriFlash 450 from Interchim. Elemental analysis experiments were performed on Thermo Electron Flash EA 1112 Series. (Arylsulfanyl)pyridine substrates (noted $\mathbf{X}, \mathbf{X}+\mathbf{1}, \ldots \mathbf{X}+\mathbf{n}$ ) were synthesized according literature reports. ${ }^{2}$

\section{Scheme S1: Molecules naming and numbering}<smiles>[R]c1ccc(Sc2cc#[R]cc2)nc1</smiles>

$\mathbf{x}$<smiles>[R][R]1cccc(Sc2c(Br)c[R4]c3cccnc23)n1</smiles>

$X d$<smiles>[R]C=C(Br)c1ncccc1Sc1c[R4]ccc1</smiles>

$\mathbf{X h}$

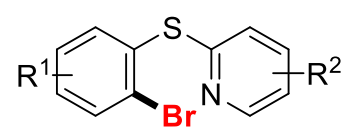

$\mathbf{X a}$

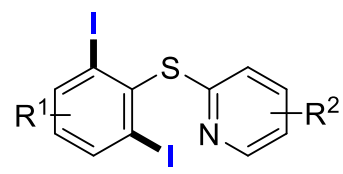

$\mathrm{Xe}$

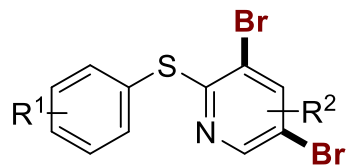

$\mathbf{X i}$<smiles>[R][R]1ccnc(Sc2c[R1]#ccc2I)c1</smiles>

$\mathbf{X b}$<smiles>[R]COc1cc[R1]cc1Sc1ccccn1</smiles>

Xf<smiles>Cc1cccnc1Sc1c(-c2ccccc2)c[R1]cc1-c1ccccc1</smiles>

19-21<smiles>[R]C=CC=C(N=C=C=C)Sc1c[R1]#ccc1Cl</smiles>

Xc

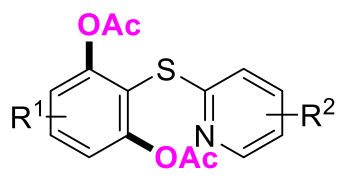

$\mathrm{Xg}$

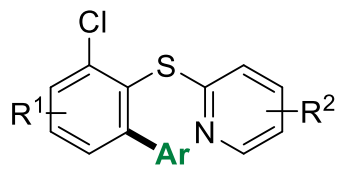

22-24 


\section{C-H bromination reaction in undried chlorobenzene}

Anhydrous conditions are essential for clean $\mathrm{C}-\mathrm{H}$ halogenation of pyridyl sulfides. To demonstrate this, we performed and monitored the reaction in undried standard chlorobenzene $(\mathrm{TCl}, 98 \%)$ as solvent [with $\mathrm{Pd}(\mathrm{acac})_{2} 10 \mathrm{~mol} \%, 3$ equiv. NBS, $120^{\circ} \mathrm{C}$ ]: after $17 \mathrm{~h}$ reaction the mixture was cooled to $-10^{\circ} \mathrm{C}$ to remove by crystallization/filtration NBS reagent excess. $\mathrm{PhCl}$ solvent was then removed by vacuum line evaporation. ${ }^{1} \mathrm{H}$ NMR spectra monitoring for this procedure is presented in Figure S1. In undried chlorobenzene solvent the reaction was found to be incomplete after $17 \mathrm{~h}$ [spectrum (d): with $65 \%$ conversion of 14, yellow square]. We observed sulfide monobromination (14a, $\nabla$ red triangle, 23\%), sulfide dibromination (14d, $\nabla$ green triangle, 15\%), and sulfoxide formation (14', $\square$ grey square, 15\%) and its brominated derivative (14'a, $\bullet$ blue circle, 11\%). The same reaction conducted under strictly anhydrous conditions (chlorobenzene 99.8\% anhydrous, Sigma-Aldrich Sure/Seal) is shown in spectrum (e) and gave a $90 \%$ conversion of 14 , for a mixture of monobrominated 14 a ( $\nabla$ red triangle, $47 \%$ ) and dibrominated $14 \mathrm{~d}$ ( $\nabla$ green triangle, 43\%) without any traces of products of sulfur oxidation.

Figure S1: ${ }^{1} \mathrm{H}$ NMR spectra (500 $\mathrm{MHz}, \mathrm{CDCl}_{3}$ ) of reagent (a), products (b, c) and mixture after $\mathrm{C}$ $H$ bromination in undried chlorobenzene (d) and under strictly anhydrous conditions (e). ${ }^{1}$

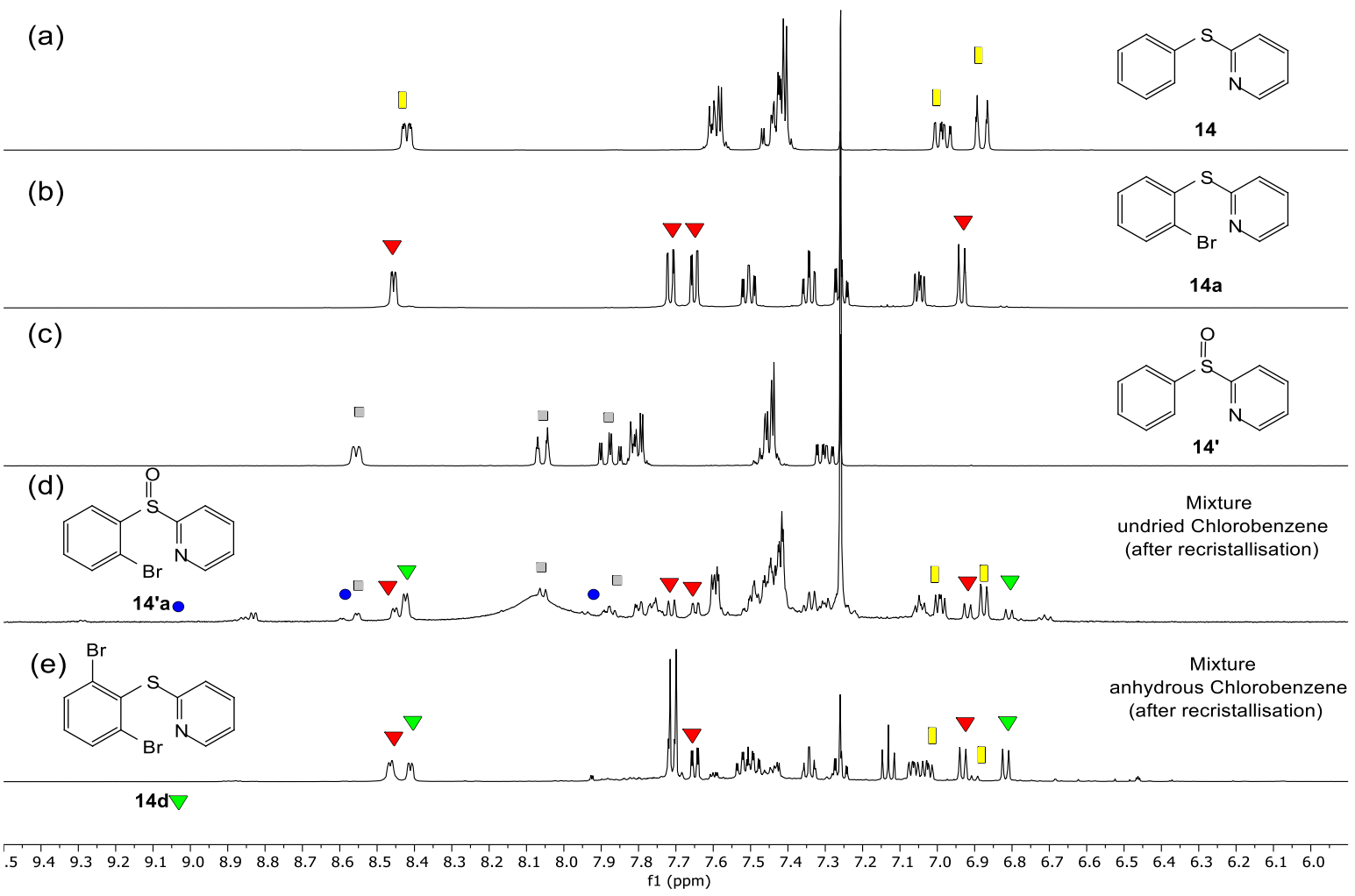

(a) 2-(phenylsulfanyl) pyridine (14, ), (b) 2-((2-bromo-phenyl)sulfanyl)-pyridine (14a, $\mathbf{\nabla})$, (c) 2-(phenylsulfoxyl) pyridine (14', $\square)$, (d) Undried PhCl with 2-((2-bromo-phenyl)sulfoxyl)-pyridine (14'a, •) formed; ${ }^{3}$ (e) Anhydrous PhCl with 2-(2,6-dibromobenzensulfanyl)pyridine $(\mathbf{1 4 d}, \mathbf{\nabla})$ formed. 
Kinetic measurements of the $\mathrm{C}-\mathrm{H}$ bromination of chlorophenyl sulfanyl pyridine (1).

Scheme S2:<smiles>Cc1cccnc1Sc1ccccc1Cl</smiles>

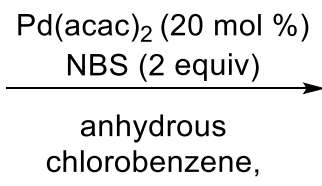

chlorobenzene

$120^{\circ} \mathrm{C}$<smiles>Cc1cccnc1Sc1c(Cl)cccc1Br</smiles>

$1 \mathrm{a}$

We monitored the course of $\mathrm{C}-\mathrm{H}$ bromination of 1 by NMR and GC. Fast conversion was revealed in the first 40 minutes, with unproductivity after 90\% conversion (Figure S2a).

Figure S2a: Graph of the kinetic study of the halogenation of 1 (to to $8 \mathrm{~h}$ ).

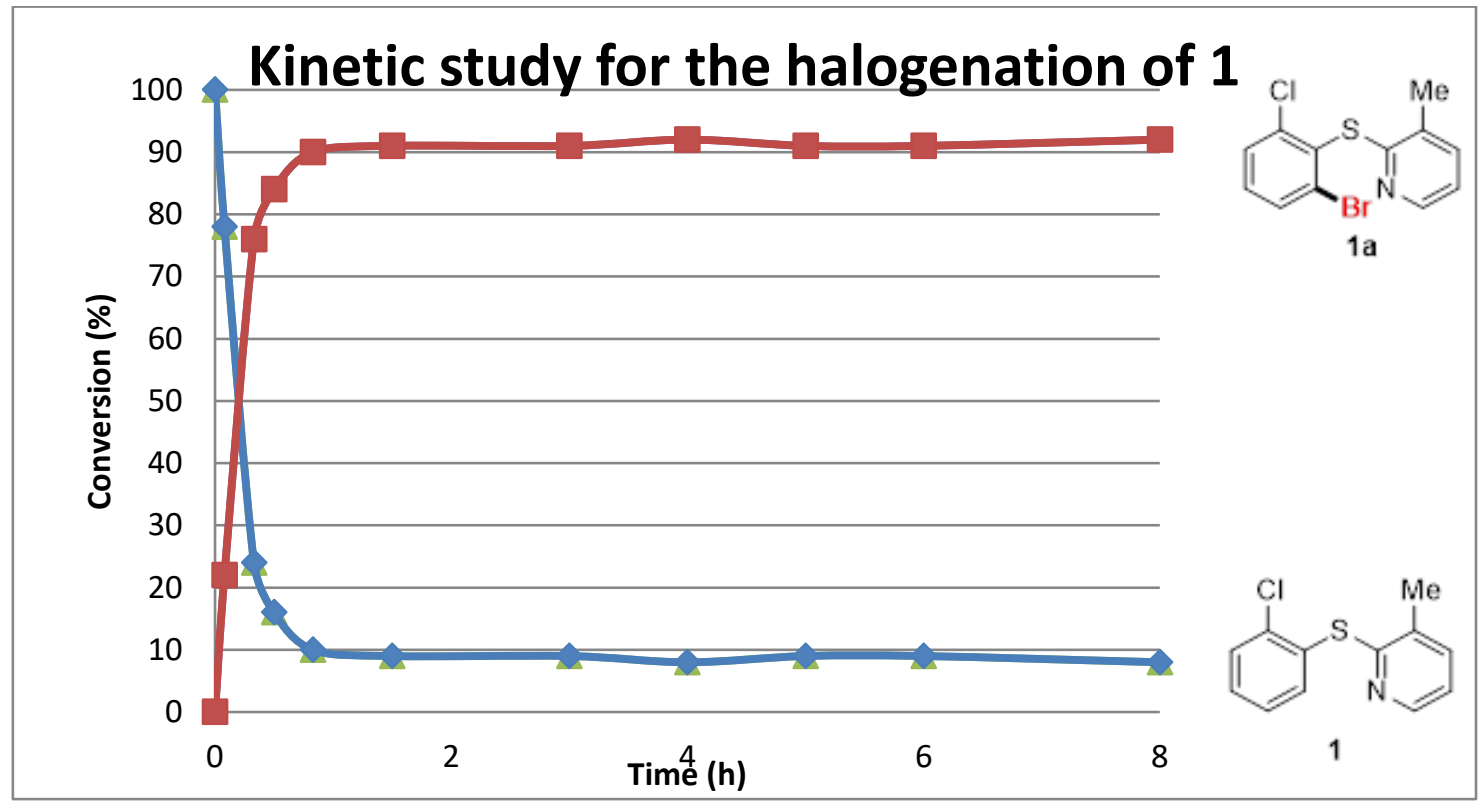

Figure S2b: Graph of the kinetic study of the halogenation of 1 (to to $90 \mathrm{~min}$ )

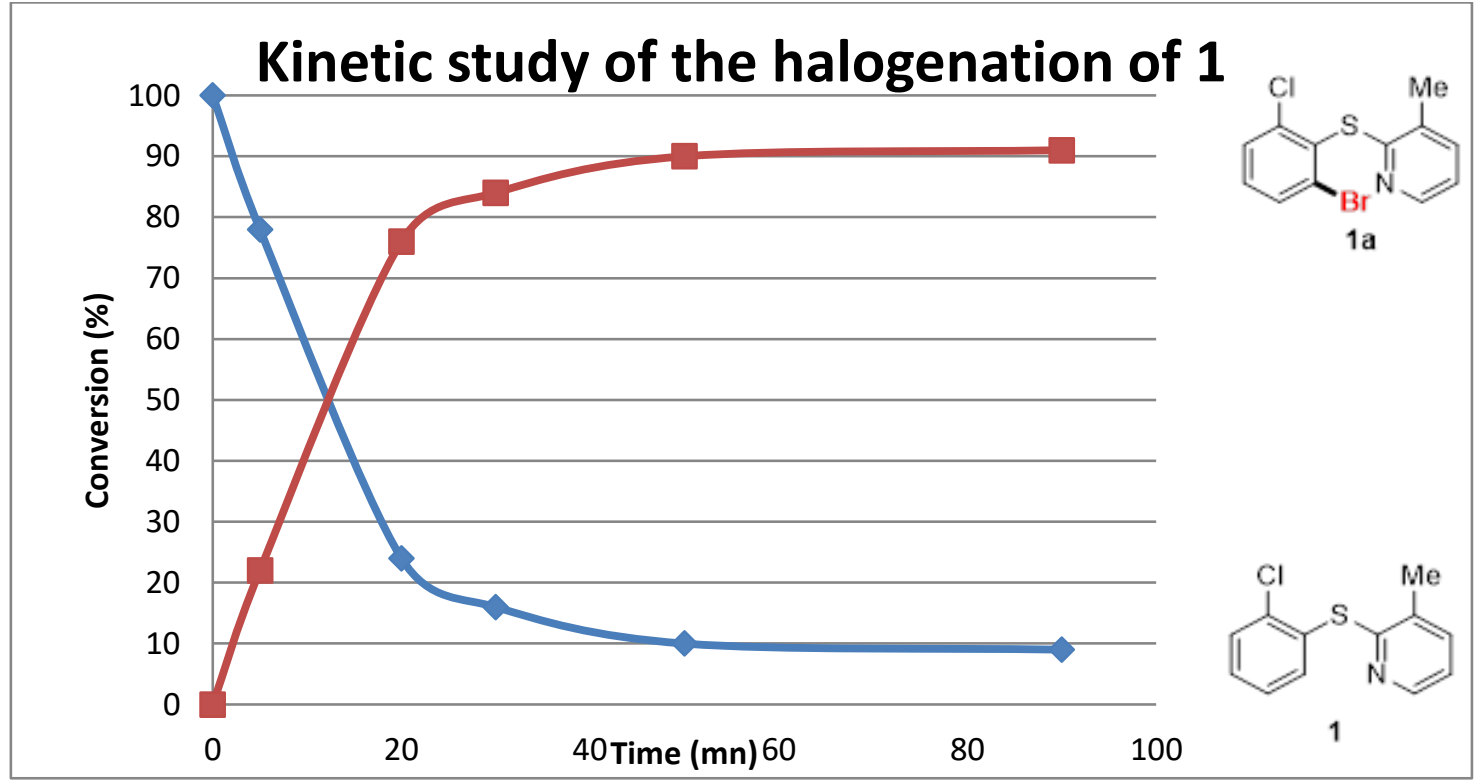


The ${ }^{1} \mathrm{H}$ NMR monitoring corresponding to kinetic studies given in Figures $\mathbf{S} 2 \boldsymbol{b}$ is presented below as Figure S2c.

Figure S2c: ${ }^{1} \mathrm{H}$ NMR monitoring of $\mathrm{C}-\mathrm{H}$ halogenation of sulfide 1 from to to $90 \mathrm{~min}$ in the aromatic protons area (500 $\left.\mathrm{MHz}, \mathrm{CDCl}_{3}\right)$.

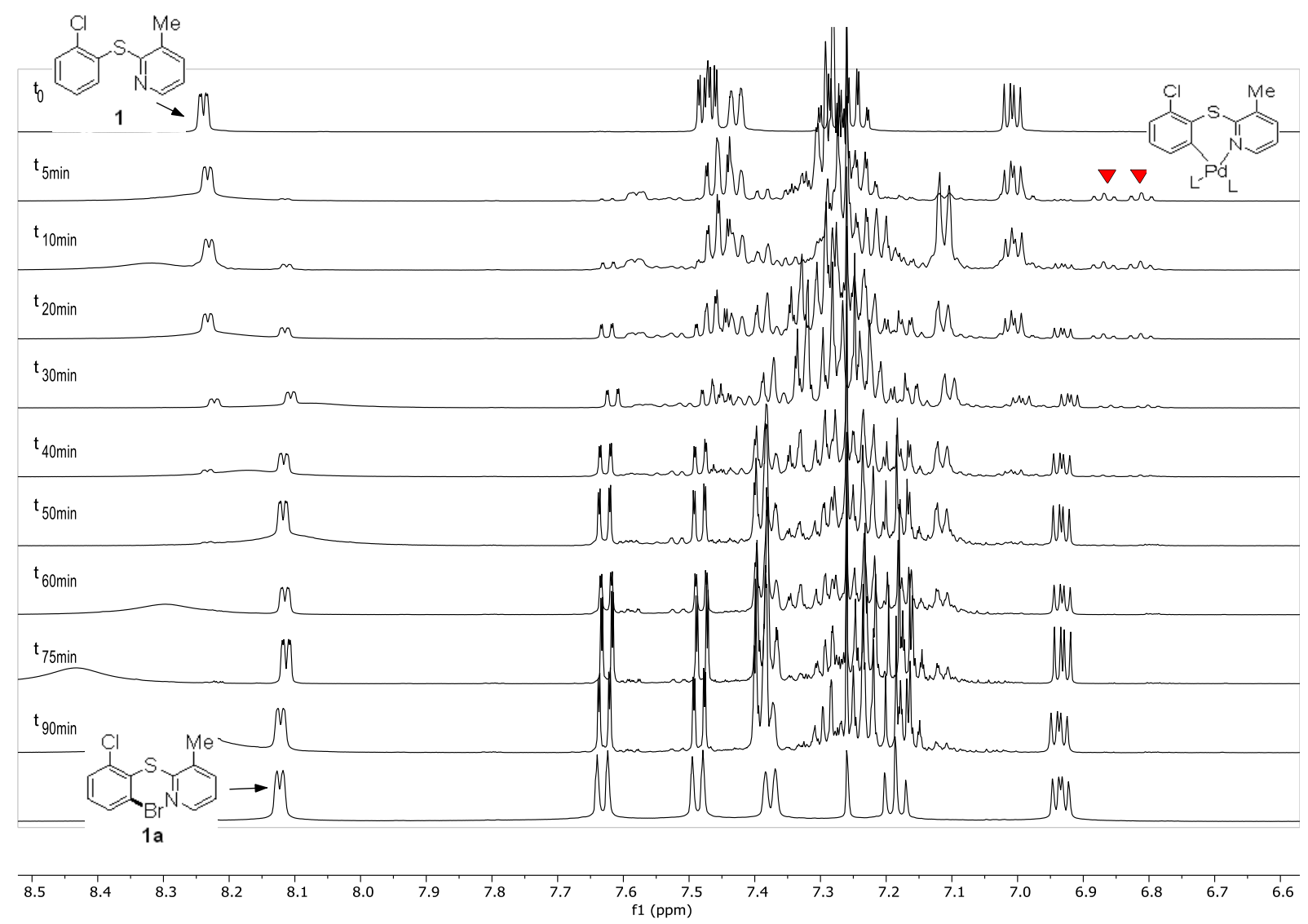

At to, only sulfide $\mathbf{1}$ is observed, with a characteristic signal at $8.25 \mathrm{ppm}$. After $5 \mathrm{~min}$, the formation of halogenated product 1a is observed (characteristic signal at $8.15 \mathrm{ppm}$ ), together with another compound showing a characteristic signal centered at $6.8 \mathrm{ppm}(\boldsymbol{\nabla}$, red triangle) that is attributed to the intermediate depicted palladacycle (top, right). Our attempts to isolate this palladacycle (or a binuclear derivative) under stoichiometric reaction condition failed, which suggested a quite unstable and reactive species. This intermediate is presumably in low concentration (albeit observable) in the first $20 \mathrm{~min}$, and was no longer easily detectable after $40 \mathrm{~min}$ (>85\% conversion of $\mathbf{1}$ to $\mathbf{1 a}$ ). 


\section{Detailed work-up procedure}

While we observed by NMR and GC that halogenated sulfides are easily formed with high conversion, their purification (like for many halogenated heteroaromatics) was found to be fairly challenging. This is also partly due to NBS excess and NHS presence, and high boiling point of chlorobenzene. We optimized the work-up procedure following the protocol detailed below for 14 .

After completion of $\mathrm{C}-\mathrm{H}$ bromination of 14 into $14 a$, the mixture was first $I)$ filtrated on a silica pad to remove palladium catalysts, ii) cooled to $-10^{\circ} \mathrm{C}$ in order to remove by crystallization/filtration the excess of NBS halide reagent and byproduct succinimide (NHS). Then, iii) $\mathrm{PhCl}$ solvent was removed by evaporation using the vacuum-line. Then, iv) the mixture was purified by silica gel column chromatography or automated flash chromatography (puriflash) to afford the brominated compounds.

We checked by NMR of the mixture the efficiency of crystallization/filtration step to remove NBS and NHS, while conserving the product 14a (Figure S3). We optimized chromatography conditions to minimize the loss of product.

\section{Scheme S3:}<smiles>c1ccc(Sc2ccccn2)cc1</smiles>

14

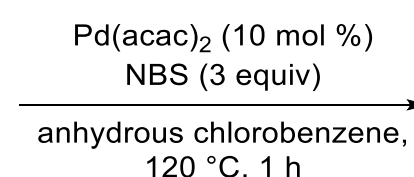
$120{ }^{\circ} \mathrm{C}, 1 \mathrm{~h}$

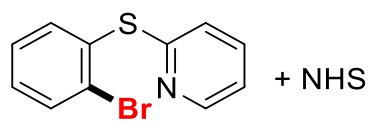

$14 a$

Figure S3: ${ }^{1} \mathrm{H}$ NMR spectra (500 $\left.\mathrm{MHz}, \mathrm{CDCl}_{3}\right)$ of $\mathrm{C}-\mathrm{H}$ bromination of 14 . (a): reagent 14; (b): mixture after (i) inorganics filtration; (c): (ii) mixture after NBS/NHS crystallization; (d): product 14a after chromatography.

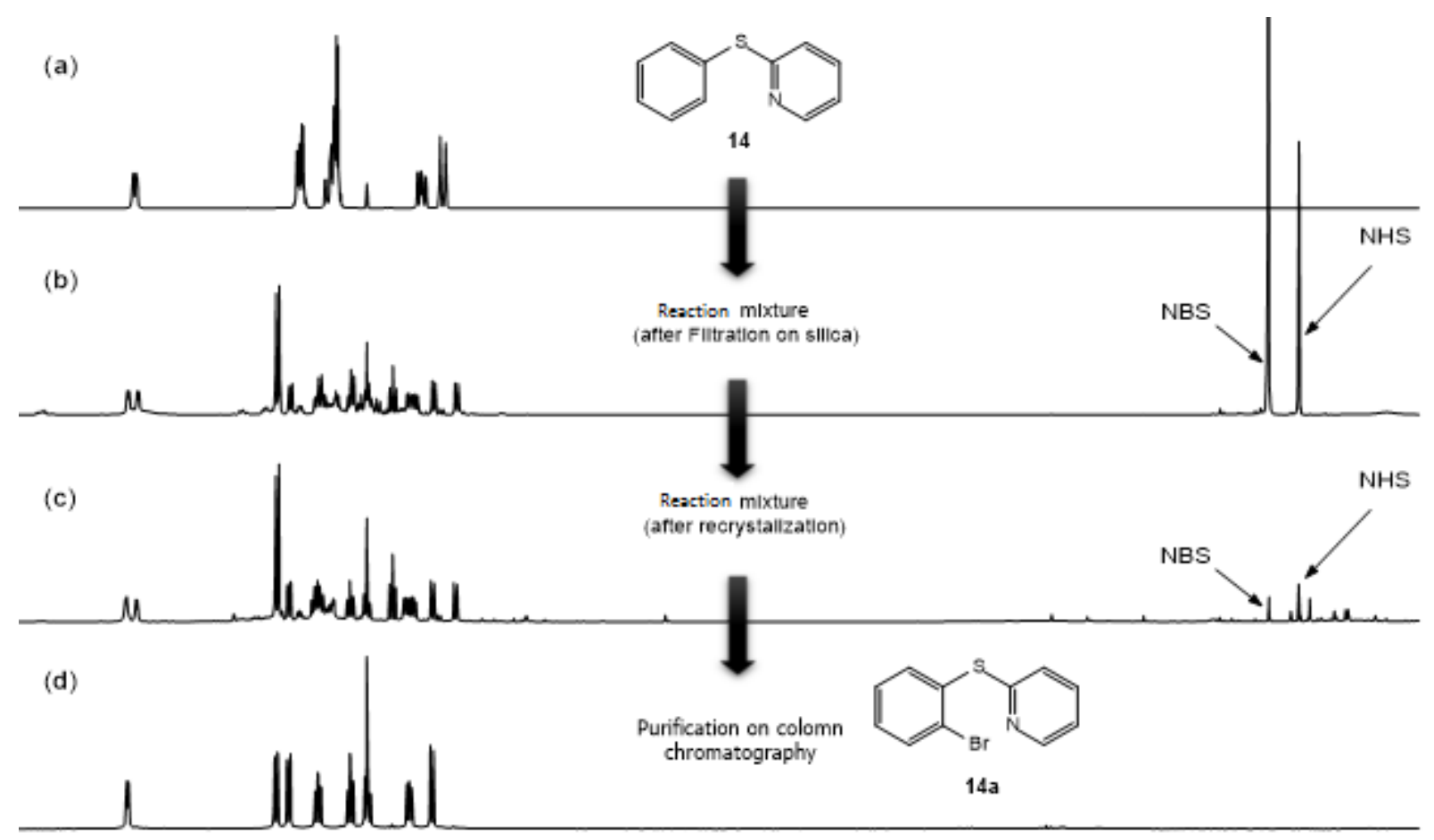

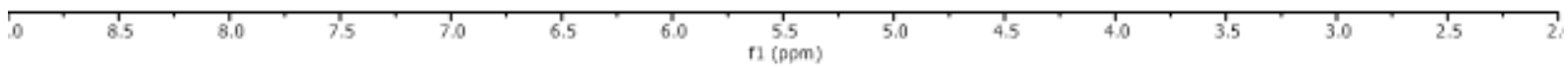




\section{Fluorination reaction of (14)}

$\mathrm{C}-\mathrm{H}$ fluorination by using $\mathrm{N}$-fluorobenzenesulfonimide (NFSI) resulted in the oxidation of sulfur atom and formed the corresponding sulfoxide (14', $\square$ grey square) and sulfone (14", $\bullet$ blue square) under the various conditions we tested (solvent, palladium precursor, oxidant amount, anhydrous media, etc.). Under our standard conditions by using NFSI (instead of NBS) the starting material 14 (, yellow square) was consumed to form in significant amount the sulfone (14", $\square$ grey square) and other unidentified compounds $(\nabla)$, presumably issued from aromatic $\mathrm{C}-\mathrm{H}$ fluorination considering the related ${ }^{19} \mathrm{~F}$ NMR $\left({ }^{1} \mathrm{H}\right.$ NMR, Figure S4a, area of ${ }^{19} \mathrm{~F}$ NMR on aromatic rings is shown in Figure S4b).

\section{Scheme S4:}<smiles>c1ccc(Sc2ccccn2)cc1</smiles>

14

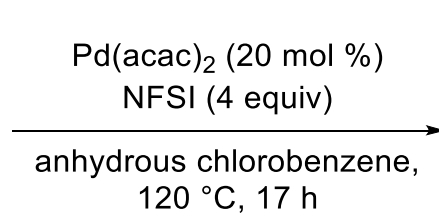

$120^{\circ} \mathrm{C}, 17 \mathrm{~h}$<smiles>O=S(=O)(c1ccccc1)c1ccccn1</smiles>

$14^{\prime}+14^{\prime \prime}$

Figure S4a: ${ }^{1} \mathrm{H}$ NMR spectra after $\mathrm{C}-\mathrm{H}$ fluorination attempt: mixture (d) compared with compounds 14 and its oxides 14' and 14" (500 MHz, $\left.\mathrm{CDCl}_{3}\right)$.

(a)

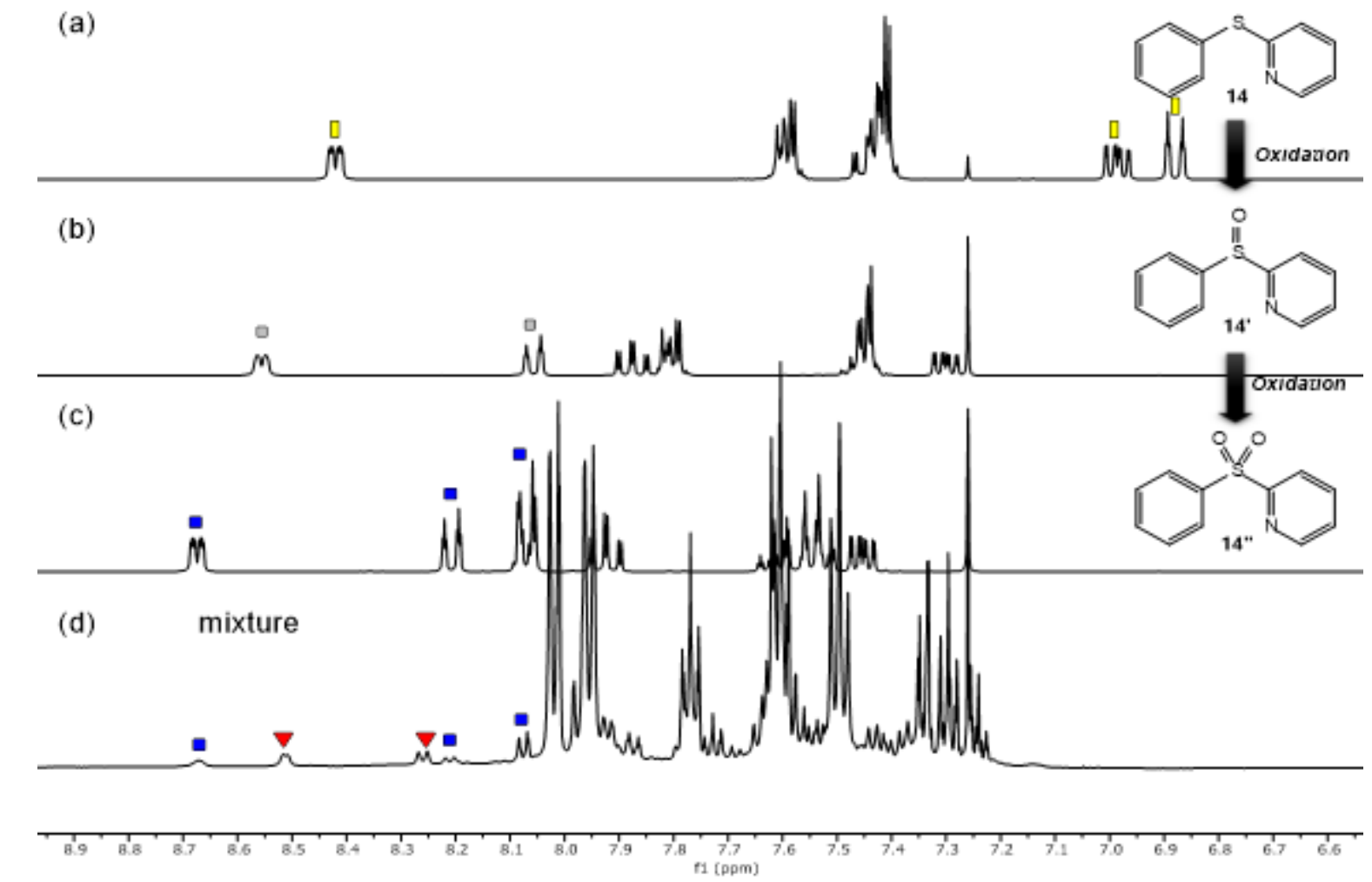

(a) 2-(phenylsulfanyl) pyridine (14, - ), (b) 2-(phenylsulfoxyl) pyridine (14', a grey square), (c) 2-(phenylsulfonyl) pyridine (14", $\square$ blue square), (d) mixture: after the filtration of Pd and of recrystallized NBS, and PhCl evaporation with 2-(phenylsulfonyl) pyridine (14", $\boldsymbol{\sim}$ blue square) and unidentified C-H fluorinated species ( $\boldsymbol{\nabla}$ ). 
${ }^{19} \mathrm{~F} \mathrm{NMR} \mathrm{(470} \mathrm{MHz}, \mathrm{CDCl}_{3}$ ) was also conducted on the mixture (see the area of $\mathrm{F}$ on aromatic rings in Figure S4b). The main signal detected was attributable to NFSI (-37.3 ppm), some product coming from its decomposition could be observed (-65.9 ppm). ${ }^{4}$ Two signals were found at -109.5 and -110.7 ppm which are tentatively attributed to aromatic $\mathrm{C}-\mathrm{H}$ fluorination of $\mathbf{1 4}$ (mono and difluorination) since the related monofluorinated sulfone (2-(2-fluorophenylsulfonyl)pyridine $)^{5}$ shows a consistent signal in ${ }^{19} \mathrm{~F}$ NMR at $-108.2 \mathrm{ppm}$.

Figure S4b: ${ }^{19} \mathrm{~F}$ NMR spectra of fluorination reaction $\left(\mathrm{CDCl}_{3}, 470 \mathrm{MHz}\right)^{1}$

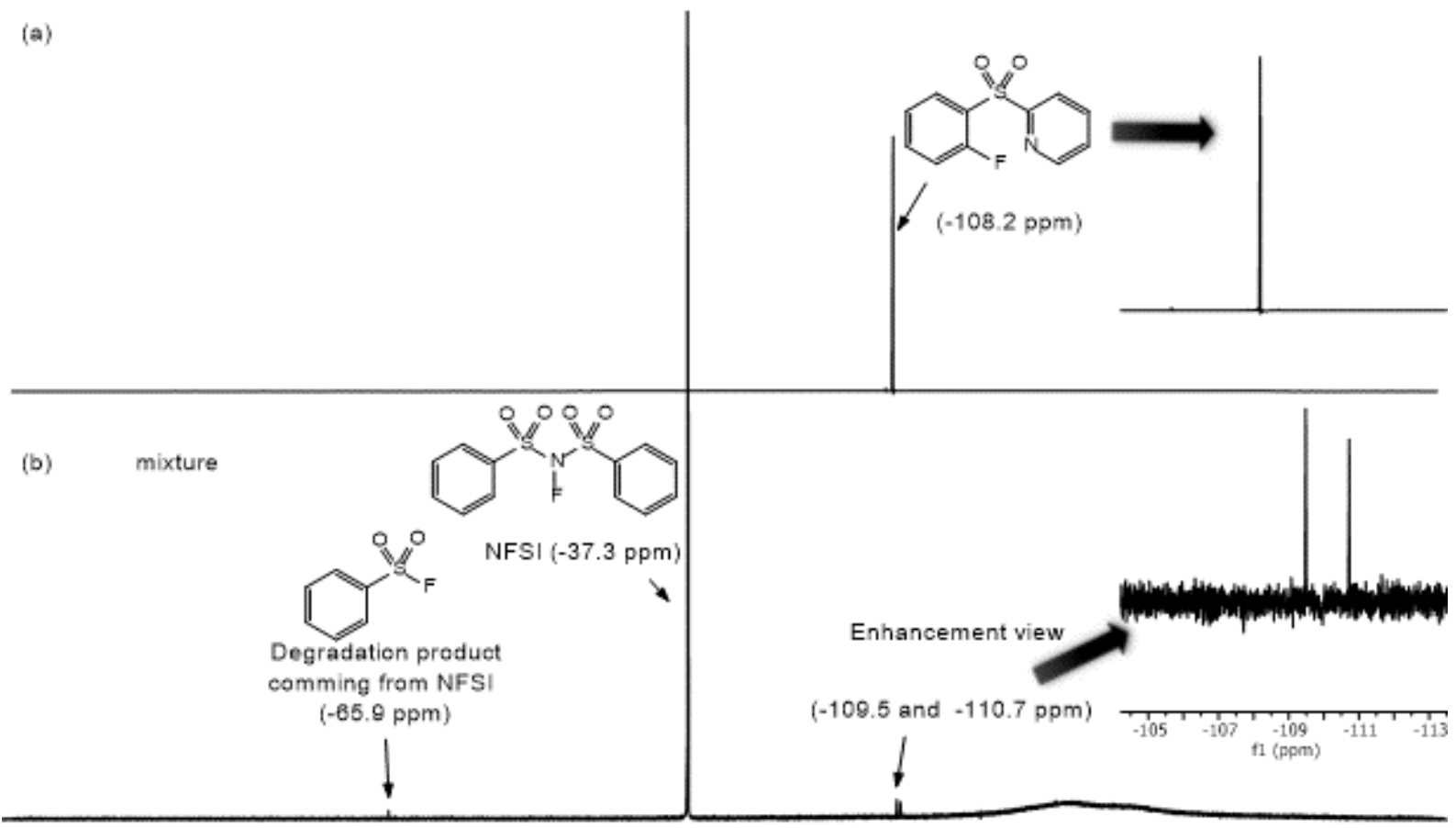

$150+10$

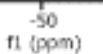

$-100$

$-150$

$-200+\frac{1}{1}+250$

(a) 2-(2-fluorophenylsulfonyl) pyridine was isolated, ${ }^{5}(\mathbf{b})$ mixture: after filtration on silica gel. 
Figures S5.1-S5.39: ${ }^{1} \mathrm{H},{ }^{13} \mathrm{C}$ and ${ }^{19} \mathrm{~F}$ NMR copy of new products

Figure S5.1: 2-(2-bromo-6-chloro-benzensulfanyl)-3-methyl-pyridine (1a):

$1 \mathrm{H}, 500 \mathrm{MHz}, \mathrm{CDCl} 3$
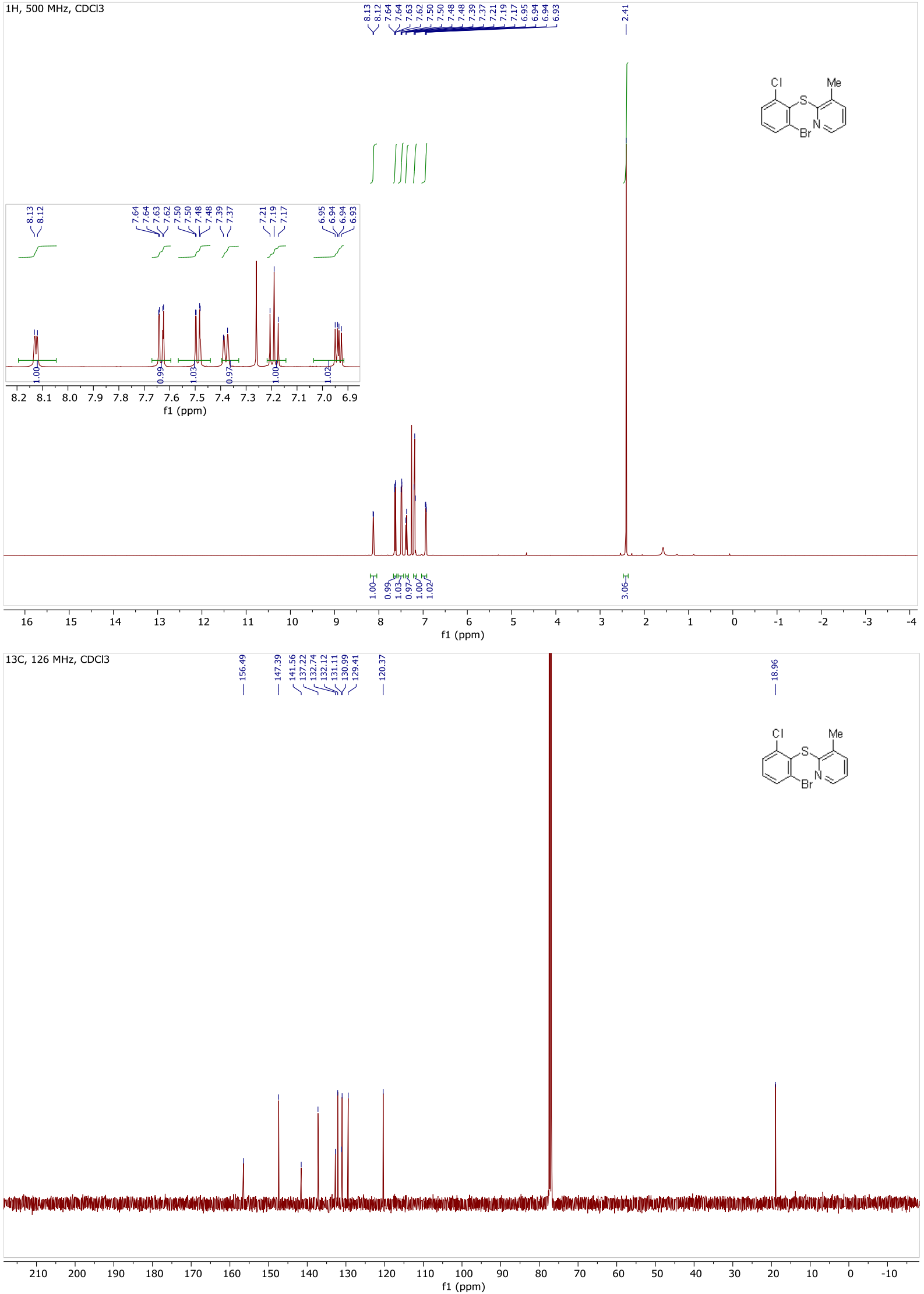
Figure S5.2: 2-((2-bromo-6-fluoro-phenyl)sulfanyl)-3-methyl-pyridine (2a):

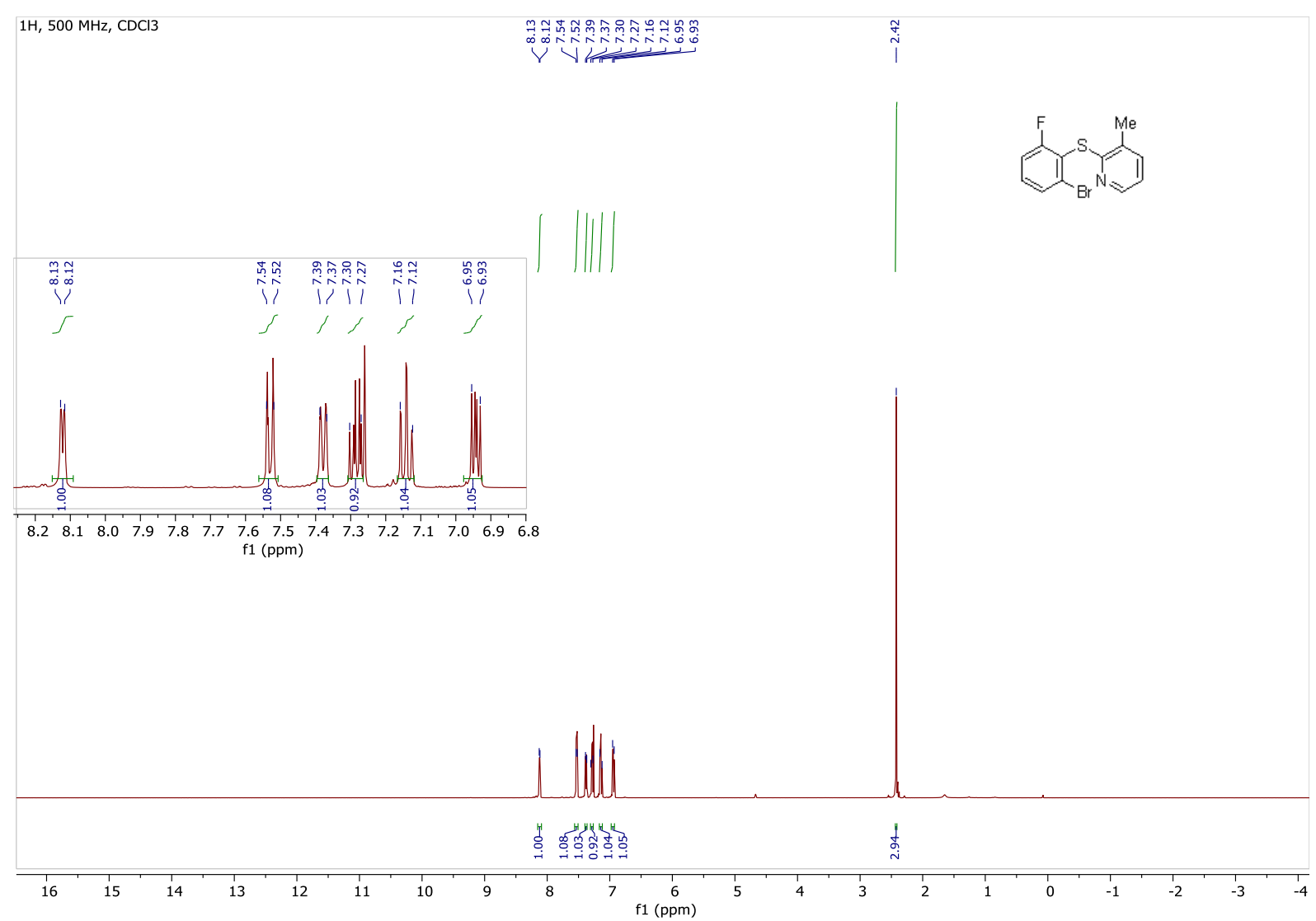

$19 \mathrm{~F}, 470 \mathrm{MHz}, \mathrm{CDCl} 3$
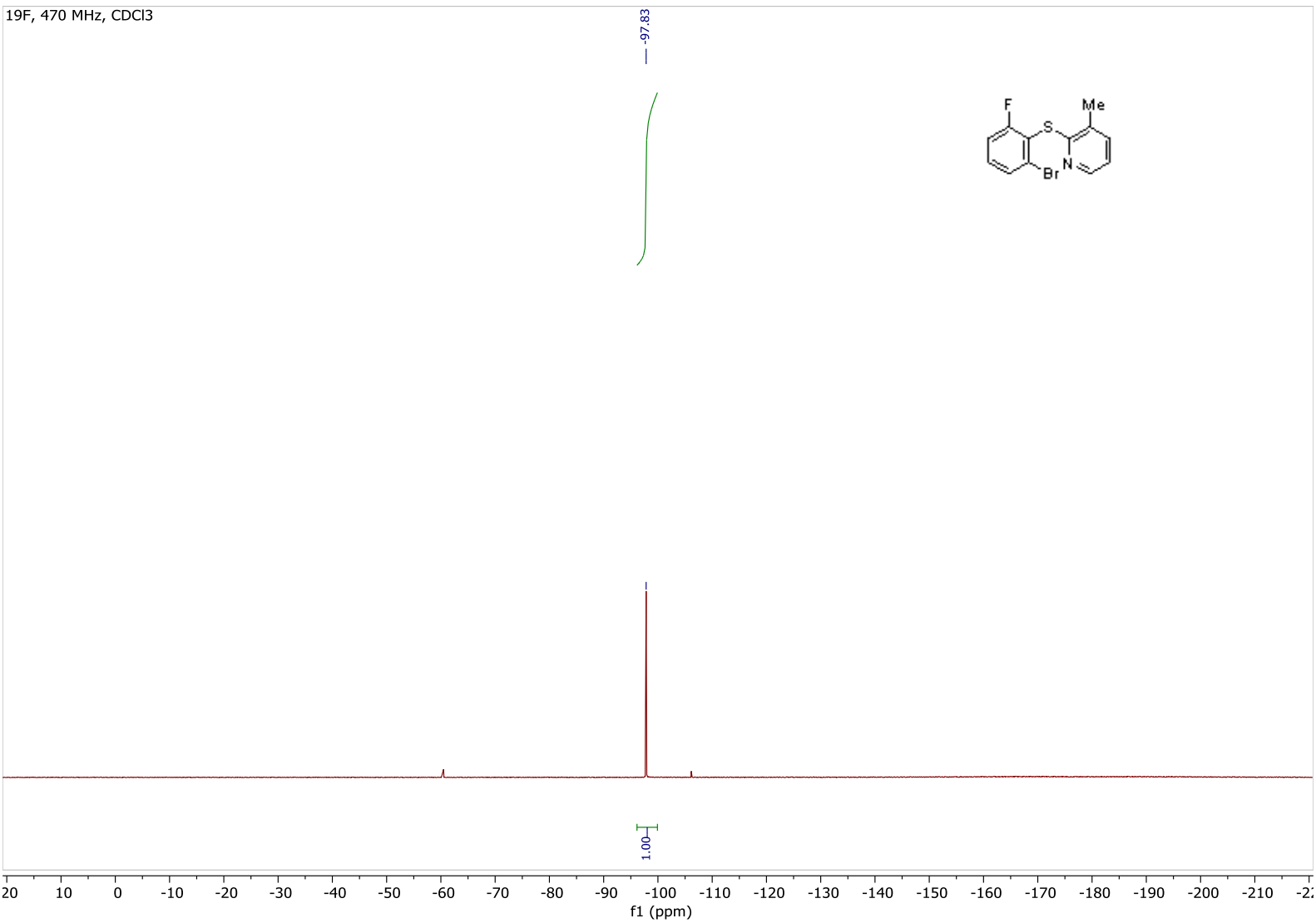


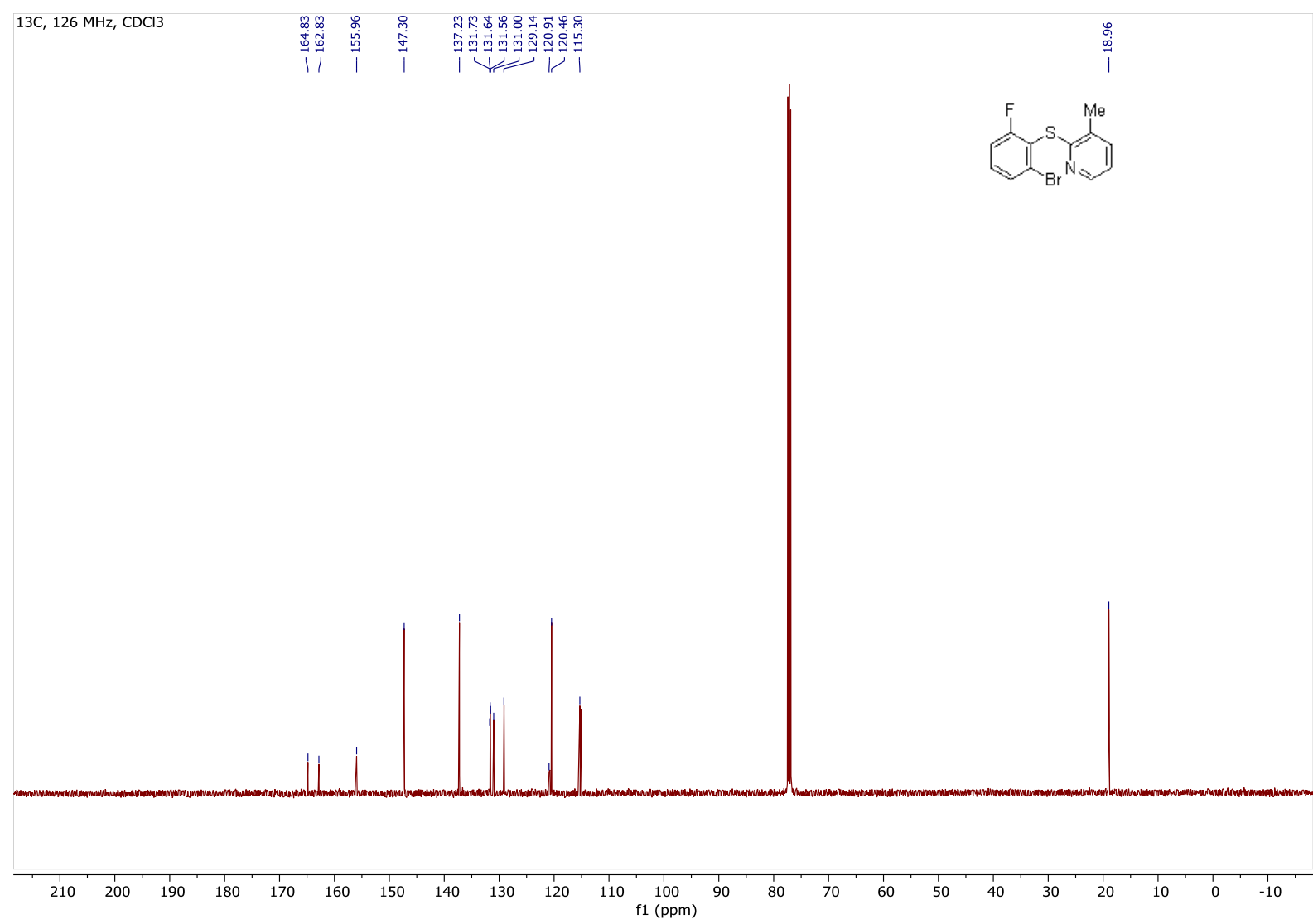

Figure S5.3: 2-(2-bromo-6-trilfuoromethyl-benzensulfanyl)-3-methyl-pyridine (3a):

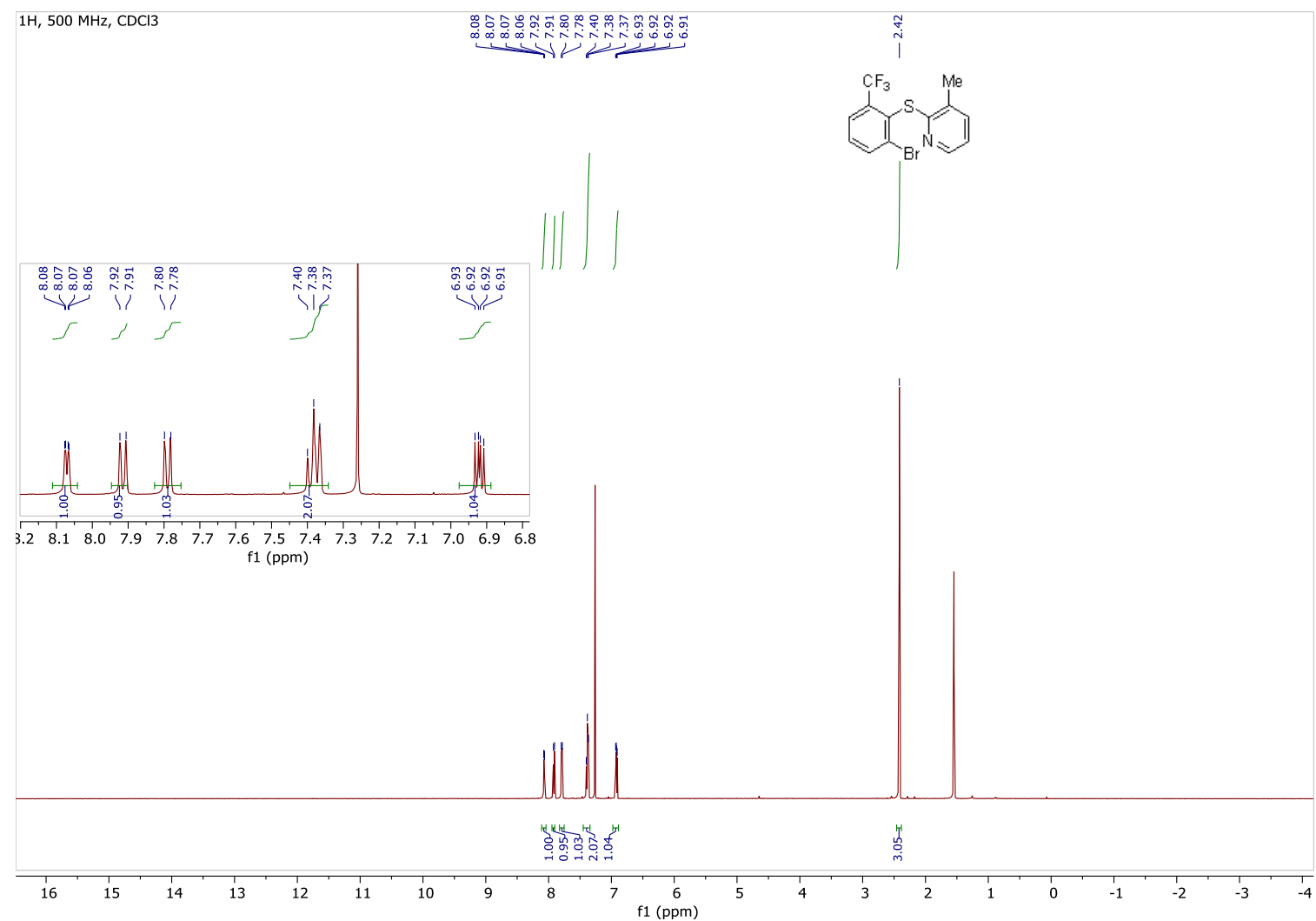




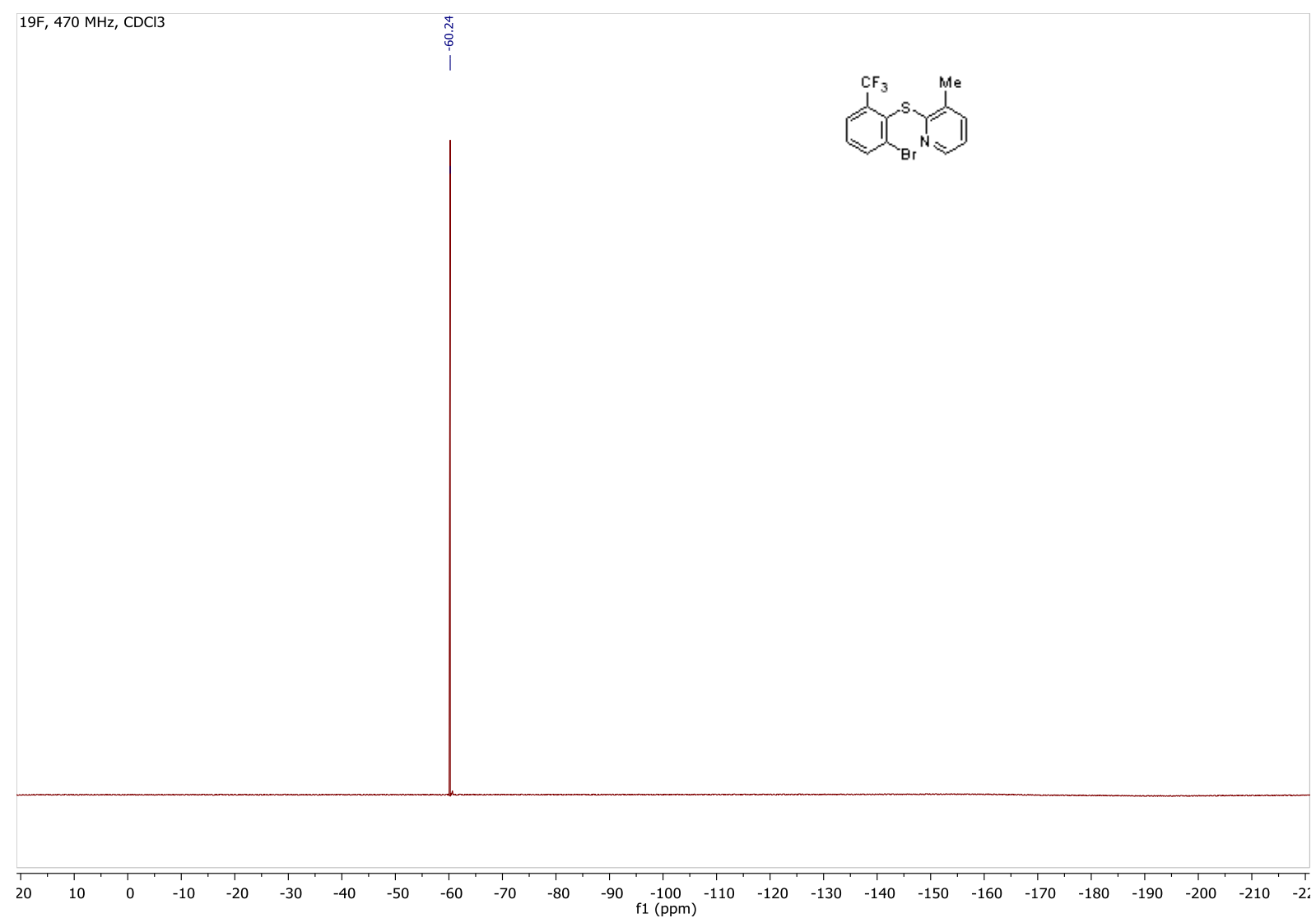

$13 \mathrm{C}, 126 \mathrm{MHz}, \mathrm{CDCl} 3$
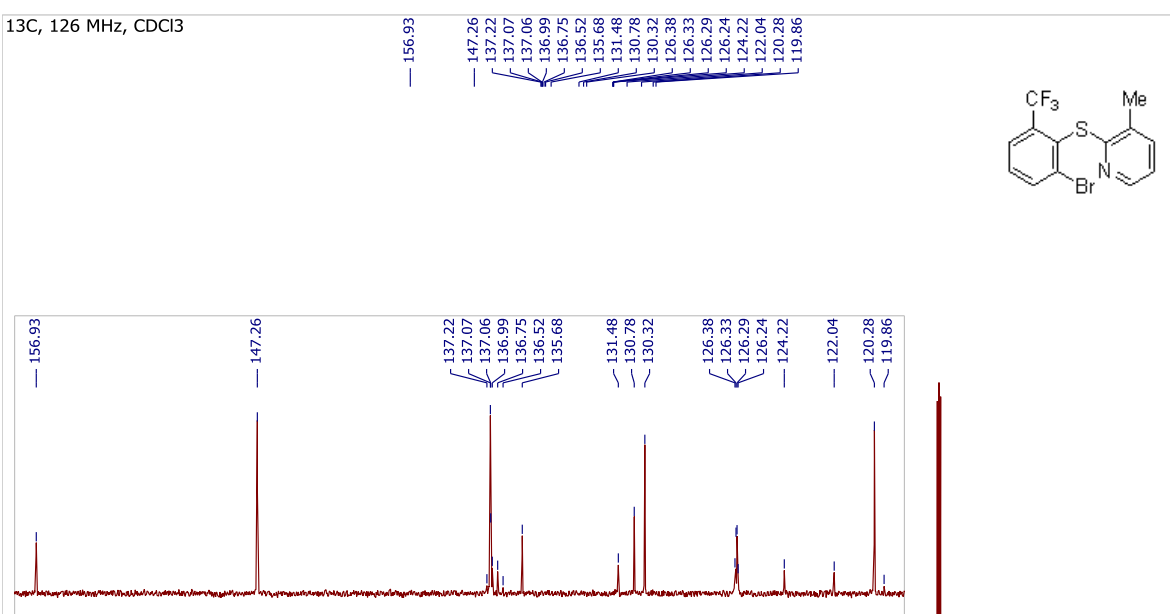

$\begin{array}{llllllllllllllllllllllllll}156 & 154 & 152 & 150 & 148 & 146 & 144 & 142 & 140 & 138 & 136 & 134 & 132 & 130 & 128 & 126 & 124 & 122 & 120\end{array}$ f1 (ppm)

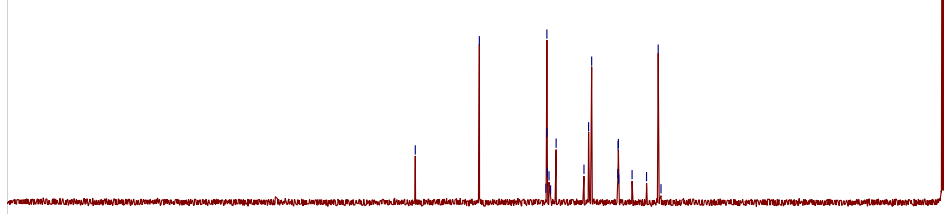

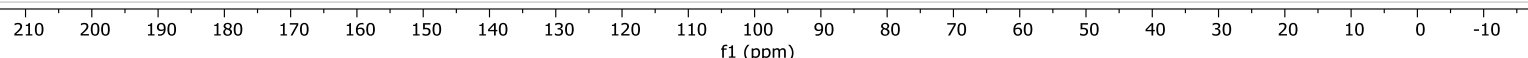


Figure S5.4: 2-(2-bromo-6-methoxy-benzensulfanyl)-3-methyl-pyridine (4a):
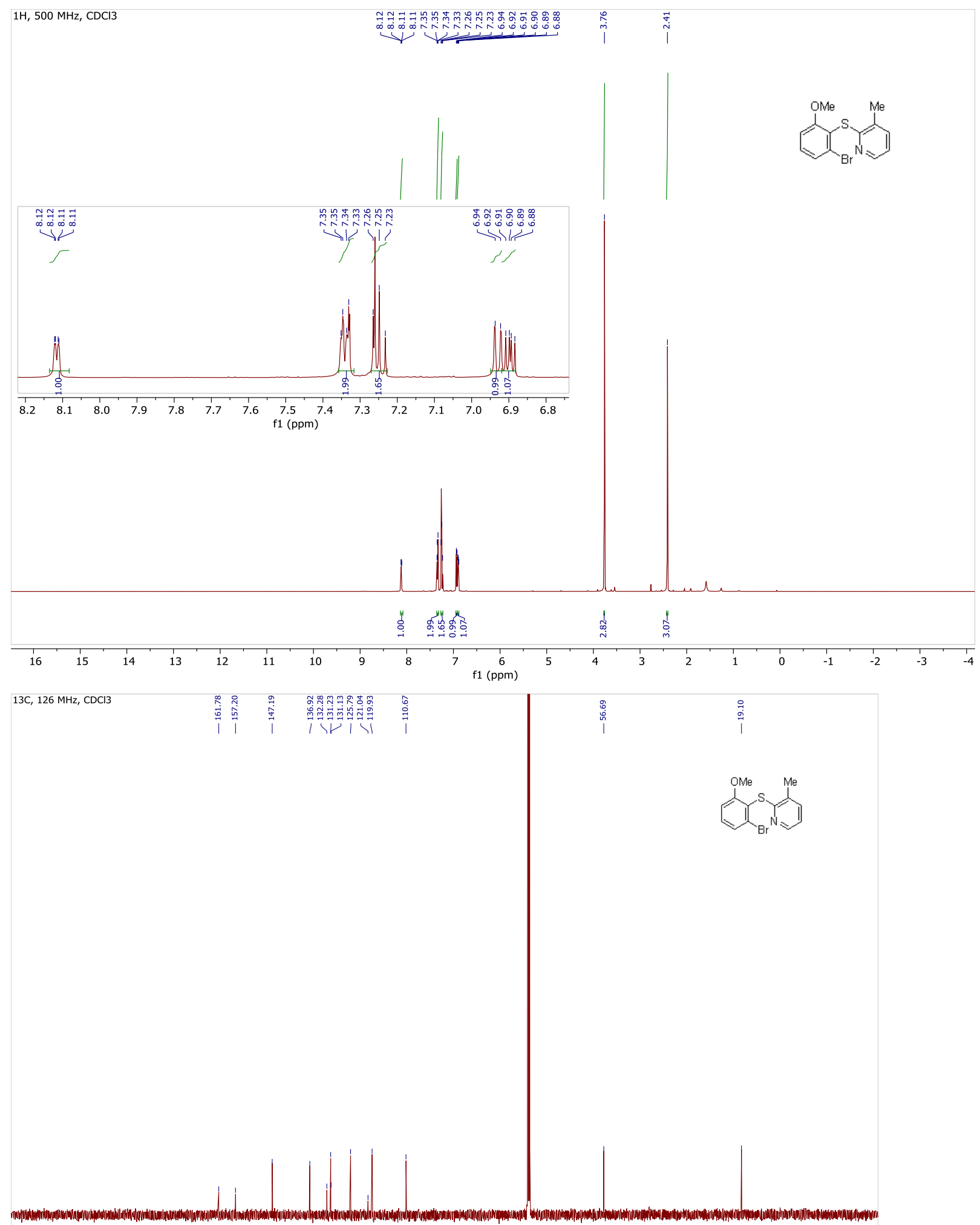

$\begin{array}{llllllllllllllllllllllllllllll}210 & 200 & 190 & 180 & 170 & 160 & 150 & 140 & 130 & 120 & 110 & 100 & 90 & 80 & 70 & 60 & 50 & 40 & 30 & 20 & 10 & 0 & -10\end{array}$ 
Figure S5.5: 2-(2-bromo-6-methyl-benzensulfanyl)-3-methyl-pyridine (5a):
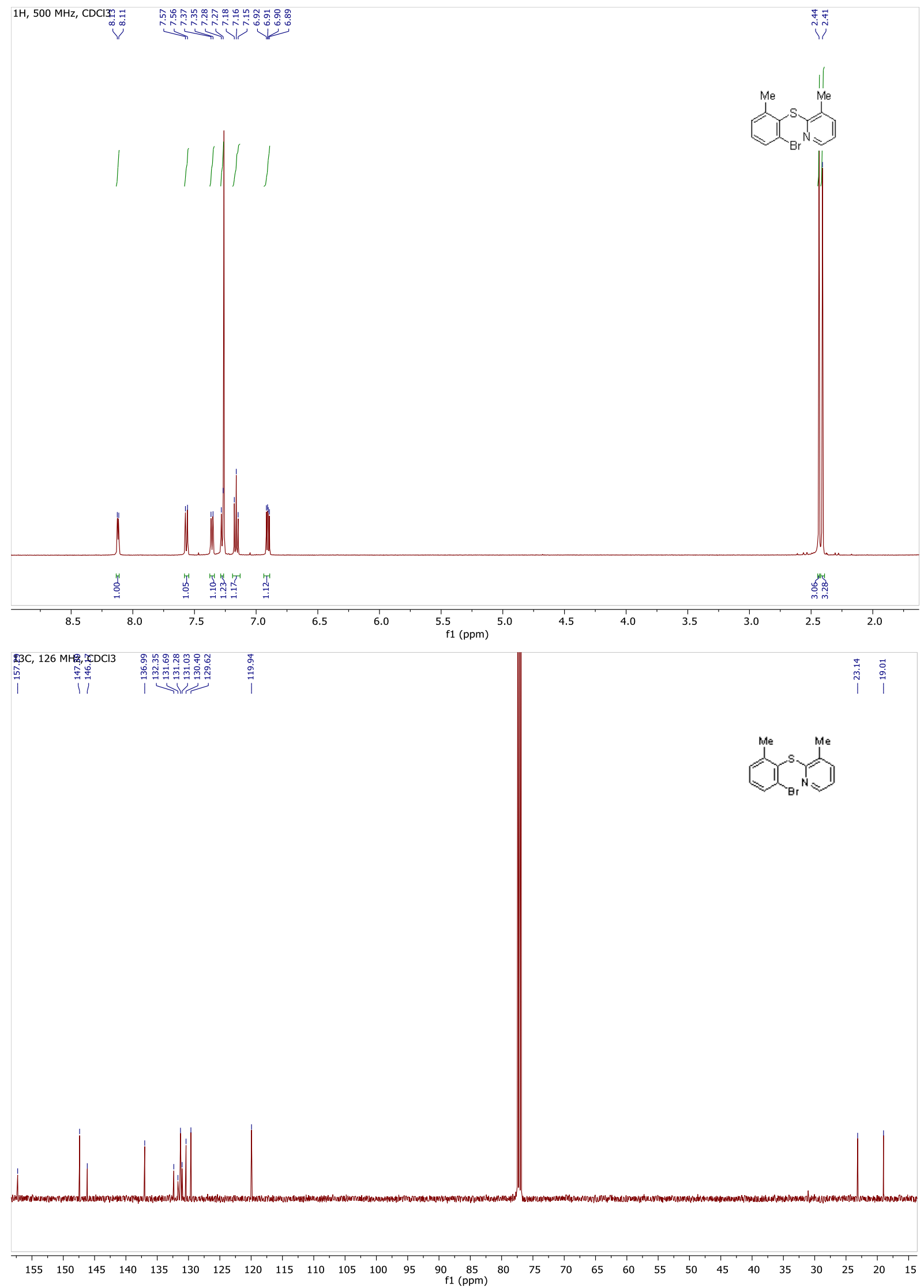
Figure S5.6: 2-(2-bromo-6-chloro-benzensulfanyl)pyridine (6a):

$1 \mathrm{H}, 500 \mathrm{MHz}, \mathrm{CDCl} 3$
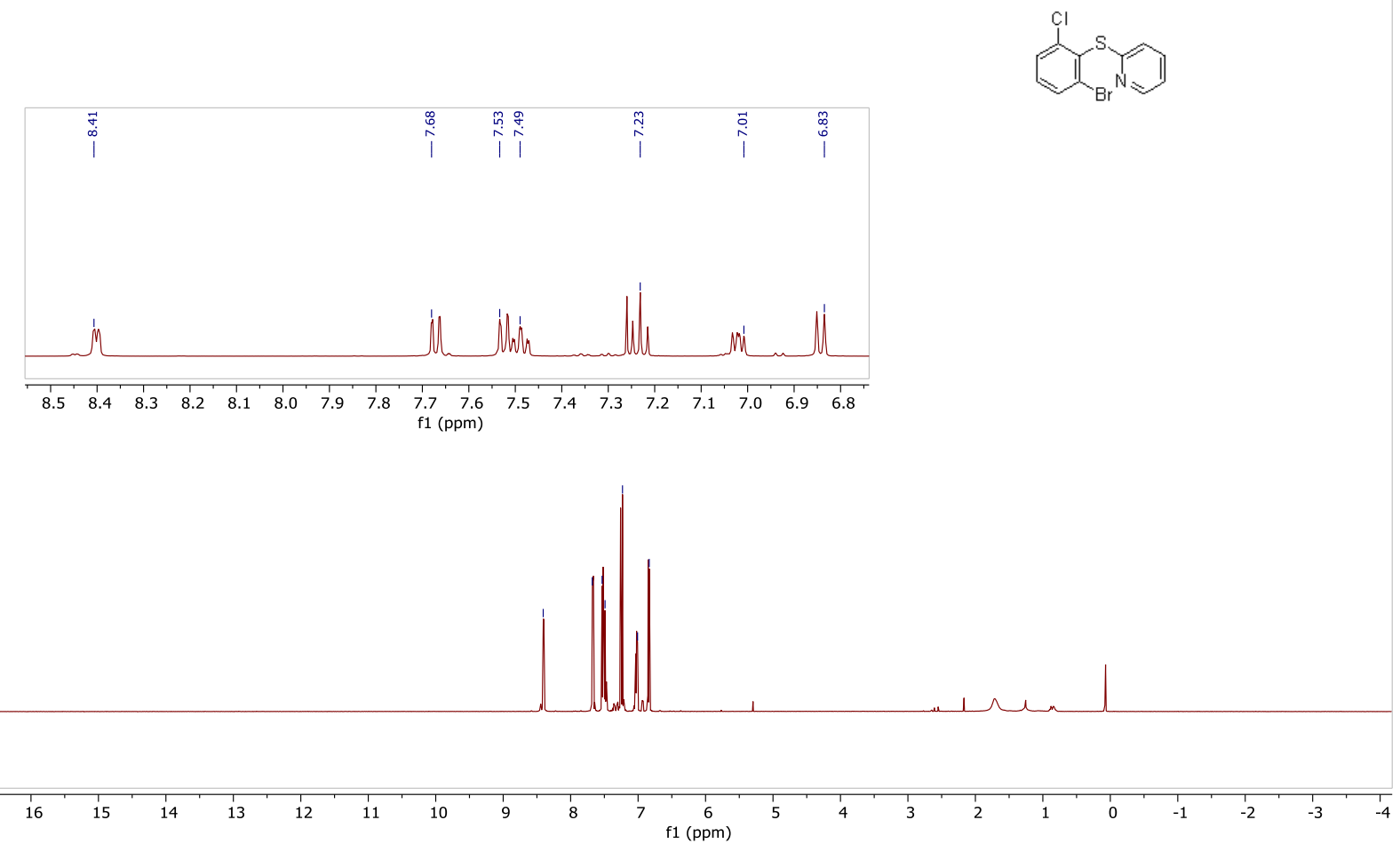

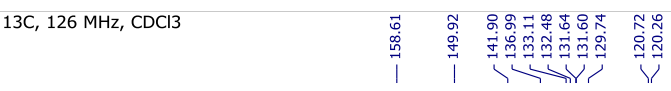
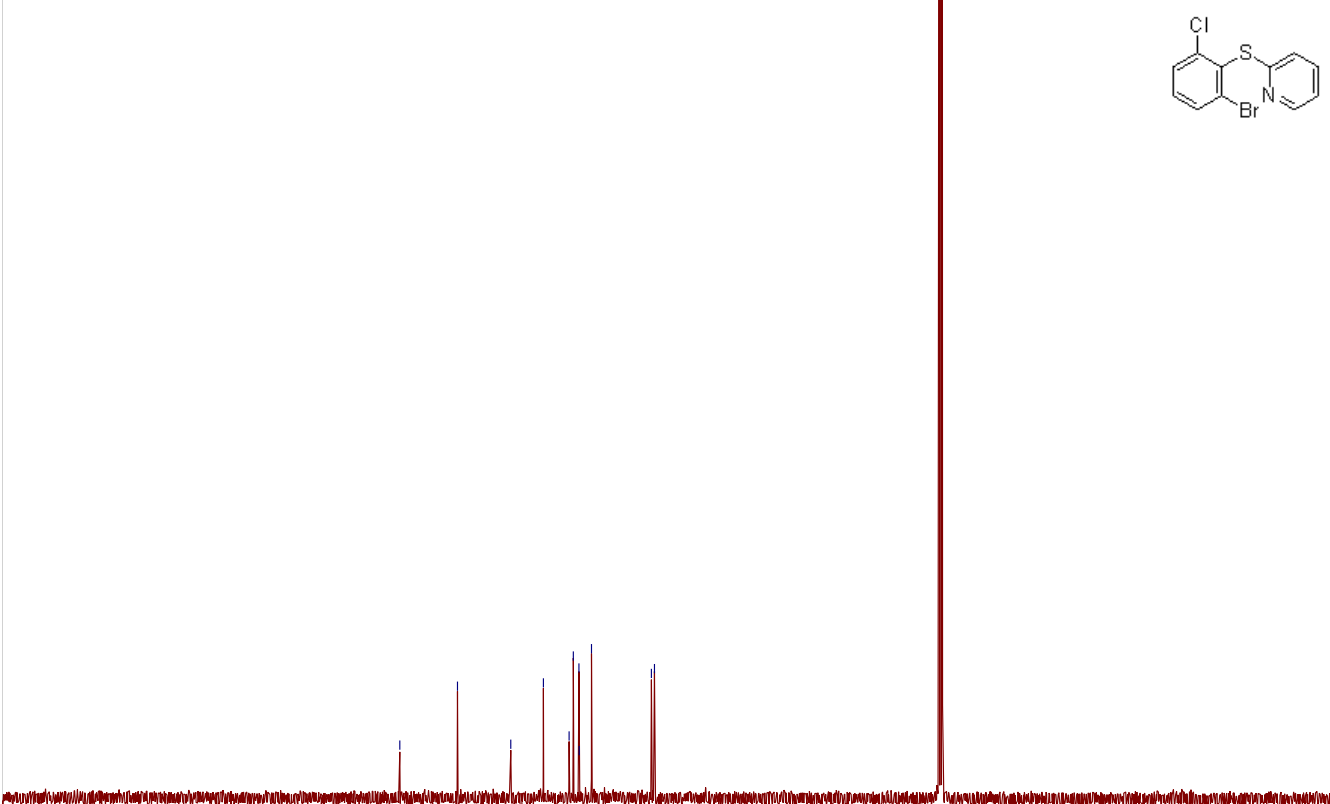

$\begin{array}{llllllllllll}210 & 200 & 190 & 180 & 170 & 160 & 150 & 140 & 130 & 120 & 110 & 100 \\ \mathrm{f} 1(\mathrm{pp})\end{array}$ 
Figure S5.7: 2-(2-bromo-6-trifluoromethyl-benzensulfanyl)pyridine (7a):
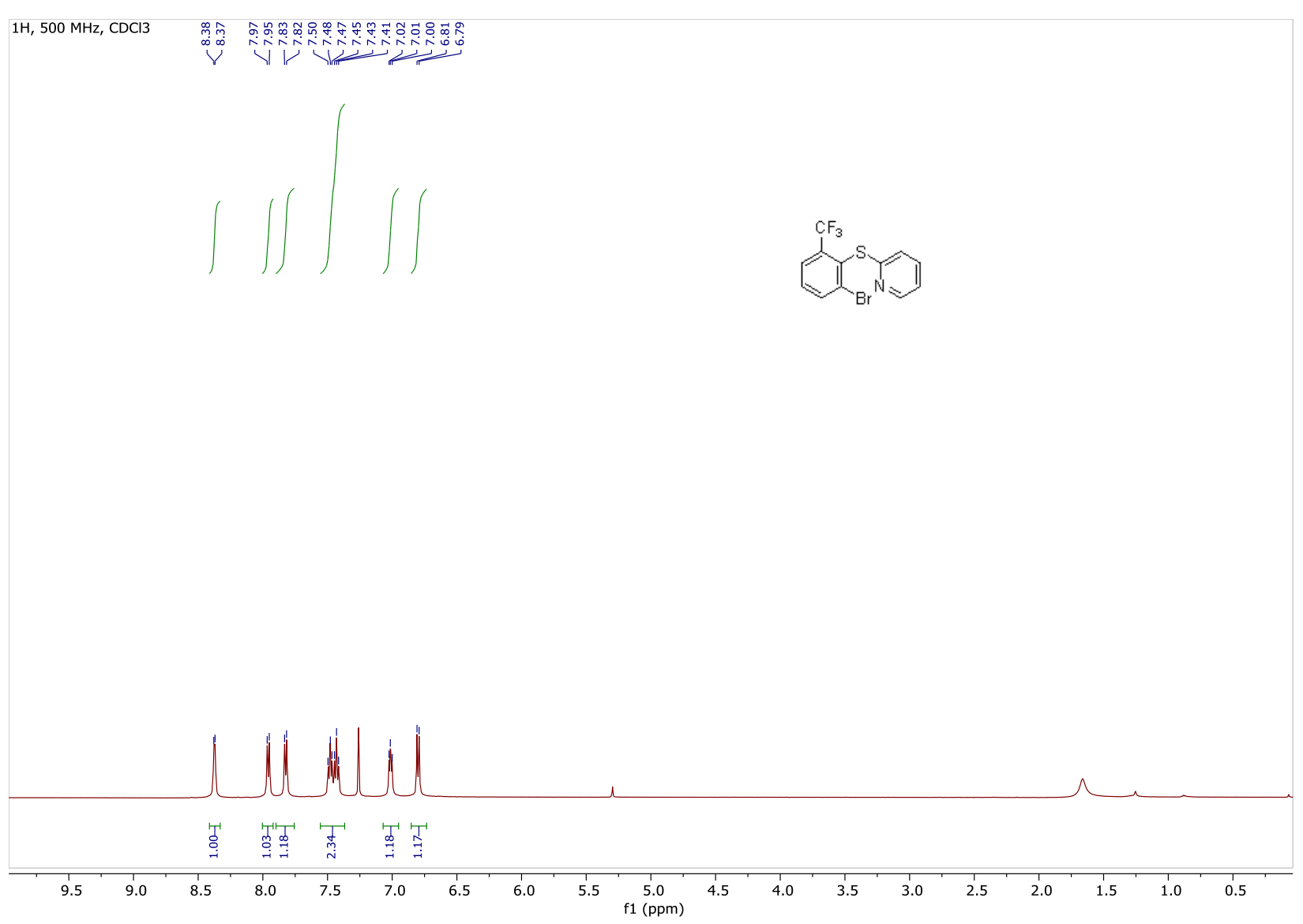

$19 \mathrm{~F}, 470 \mathrm{MHz}, \mathrm{CDCl} 3$
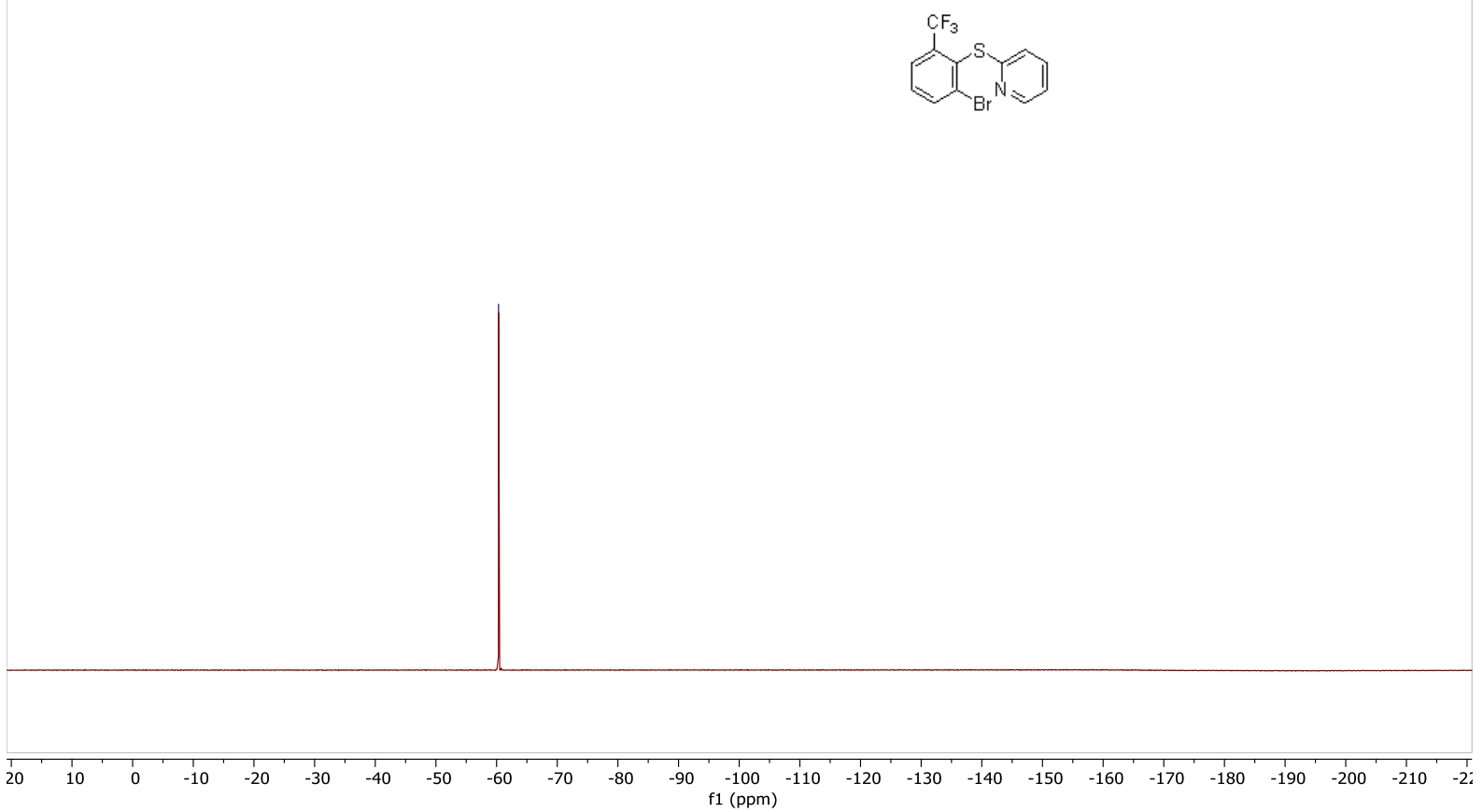

S16 

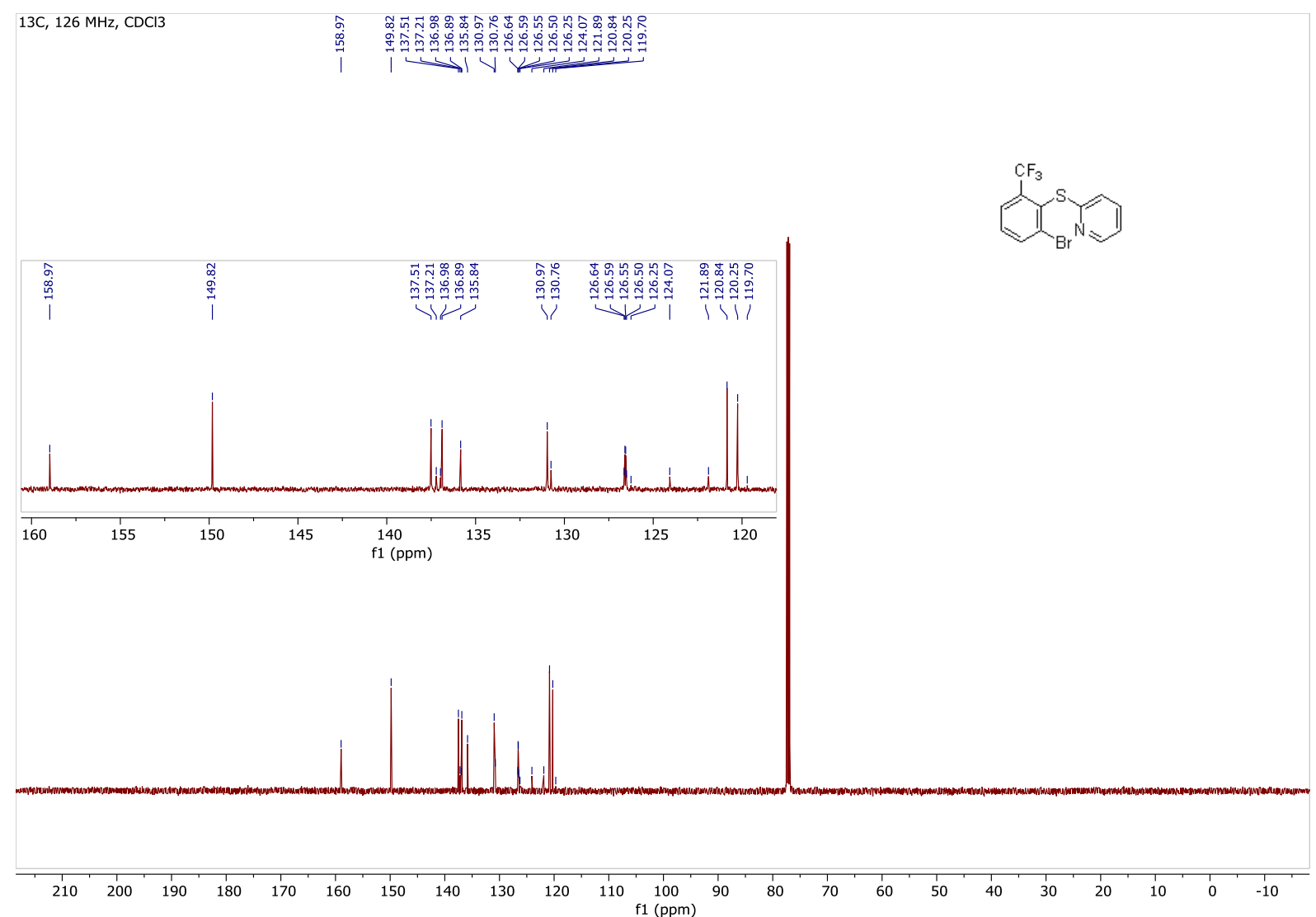
Figure S5.8: 2-(2-bromo-6-methoxy-benzensulfanyl)pyridine (8a):
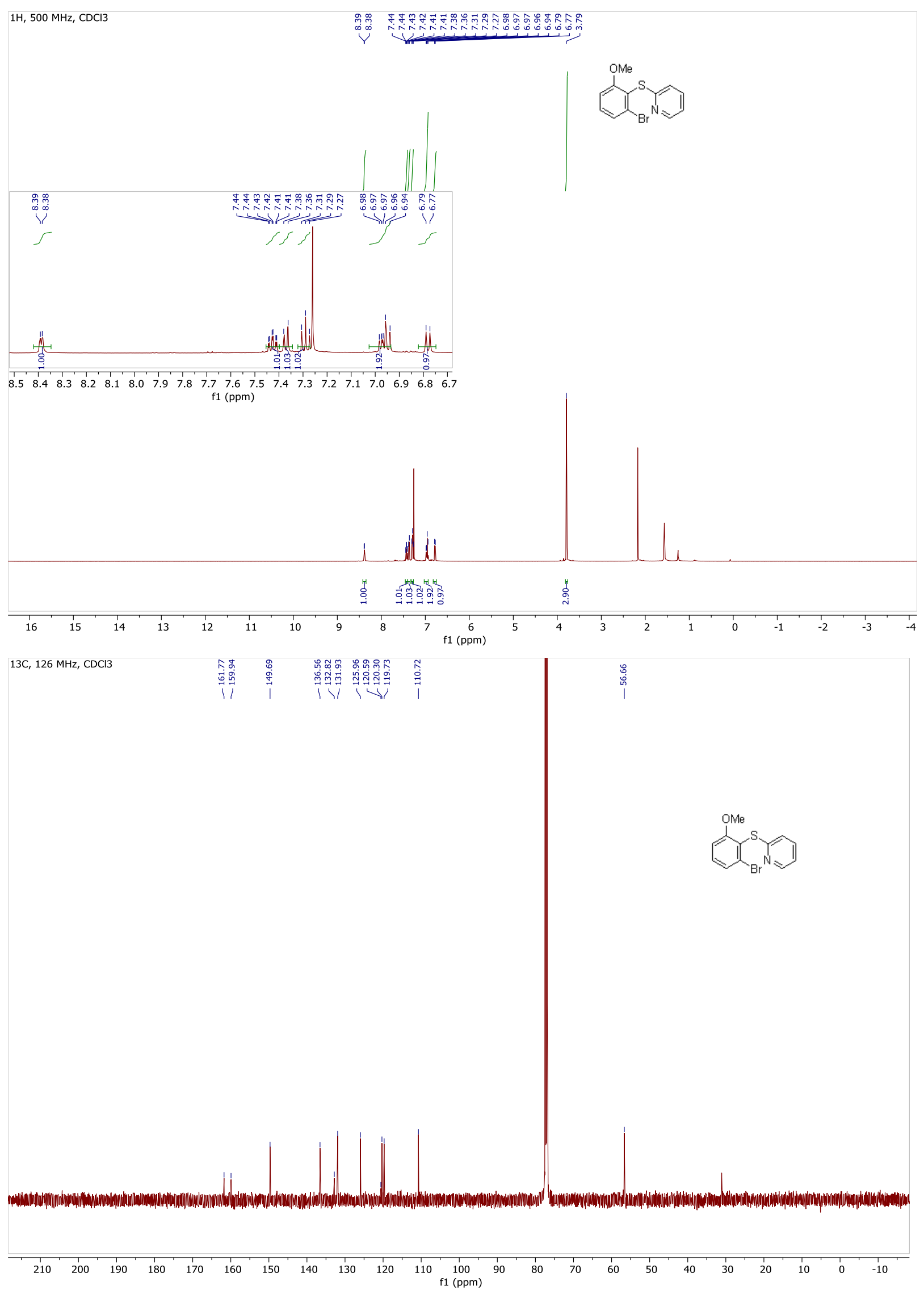

S18 
Figure S5.9: 2-((2-iodo-6-chloro-phenyl)sulfanyl)-3-methyl-pyridine (1b):

$1 \mathrm{H}, 500 \mathrm{MHz}, \mathrm{CDCl} 3$
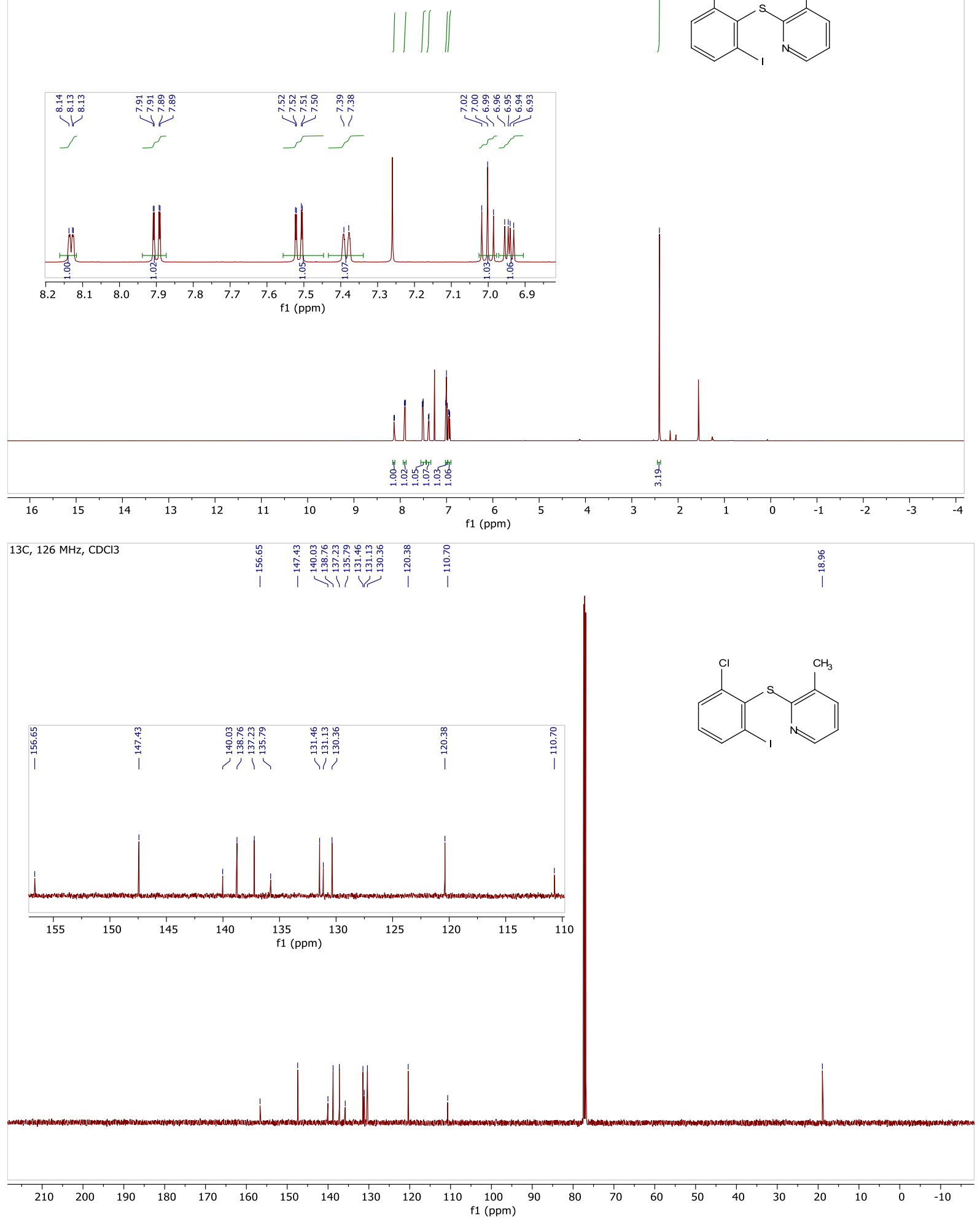

S19 
Figure S5.10: 2-((2-iodo-6-trifluoromethyl-phenyl)sulfanyl)-3-methyl-pyridine (3b):

$1 \mathrm{H}, 500 \mathrm{MHz}, \mathrm{CDCl} 3$
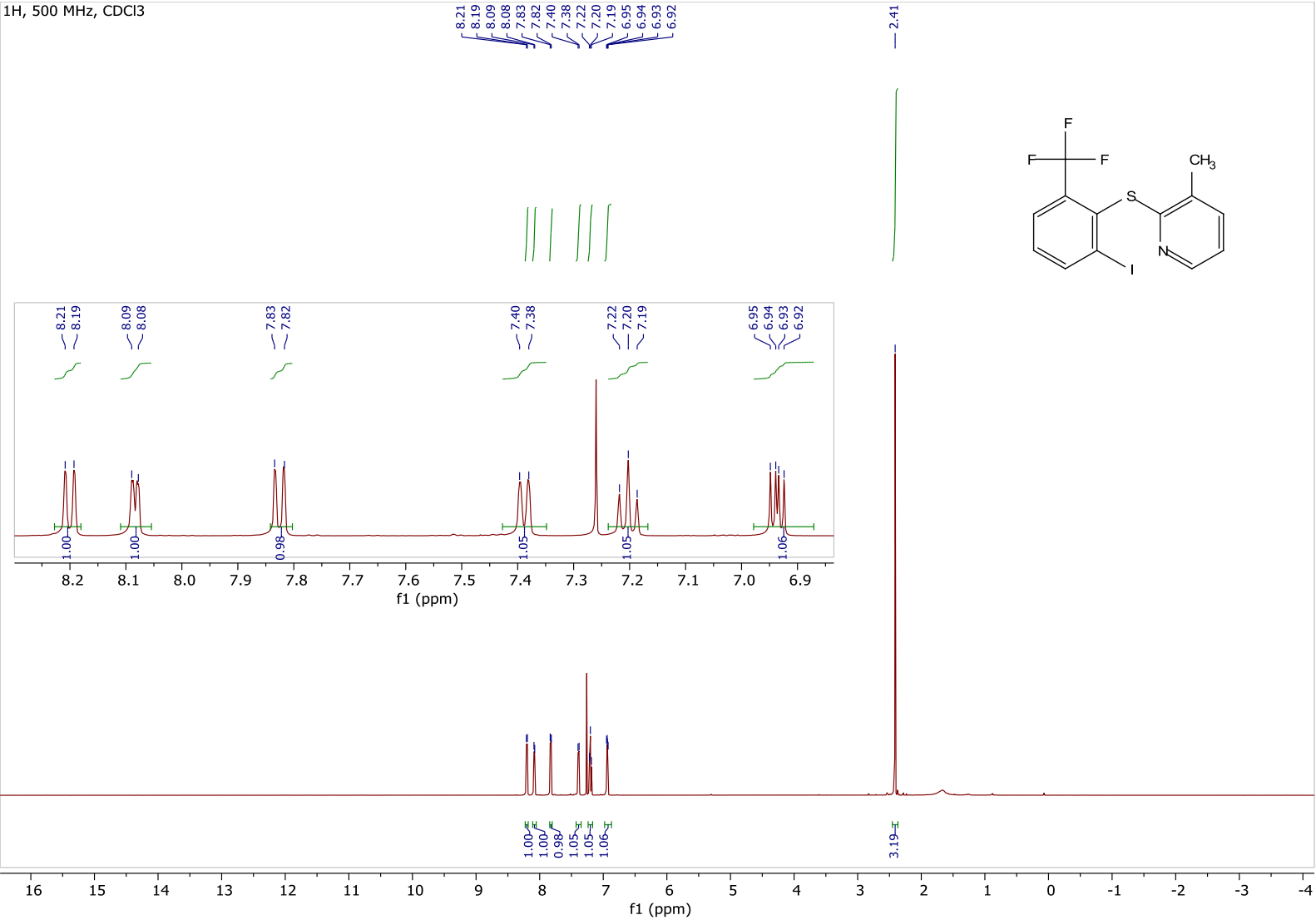

$19 \mathrm{~F}, 470 \mathrm{MHz}, \mathrm{CDCl} 3$
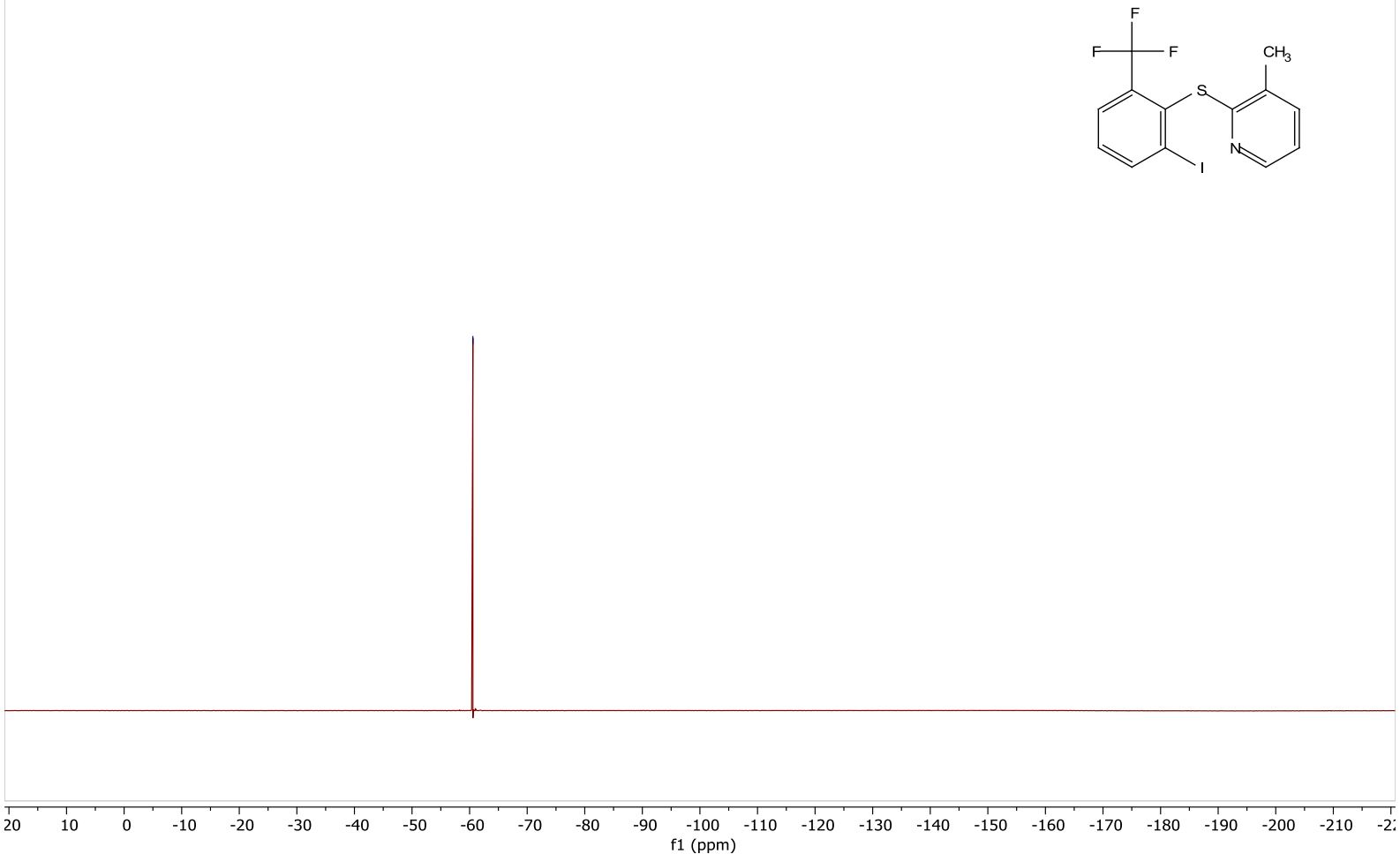


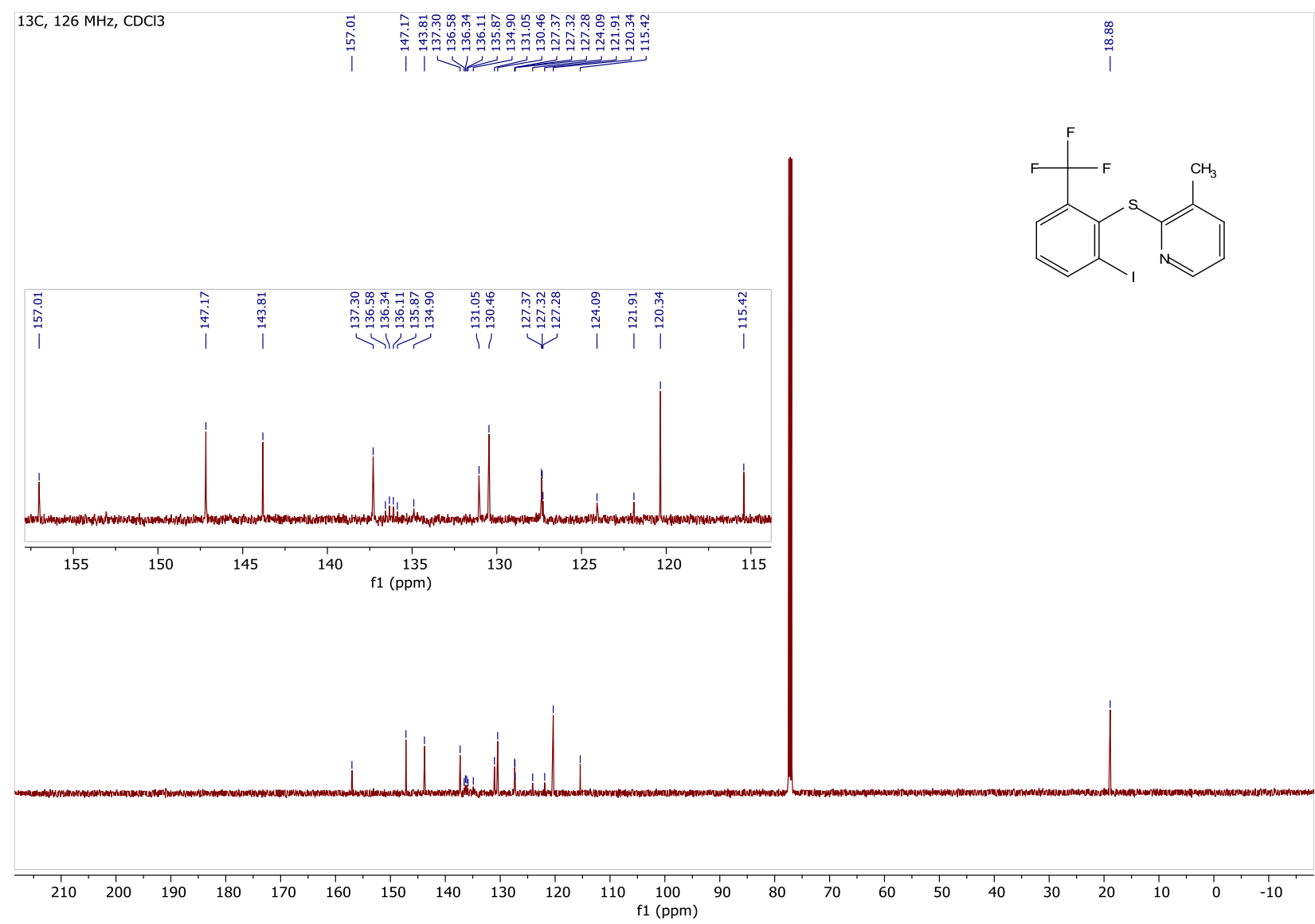


Figure S5.11: 2-((2-iodo-6-methoxy-phenyl)sulfanyl)pyridine (4b):

$1 \mathrm{H}, 500 \mathrm{MHz}, \mathrm{CDCl} 3$

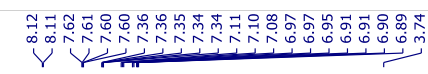

I
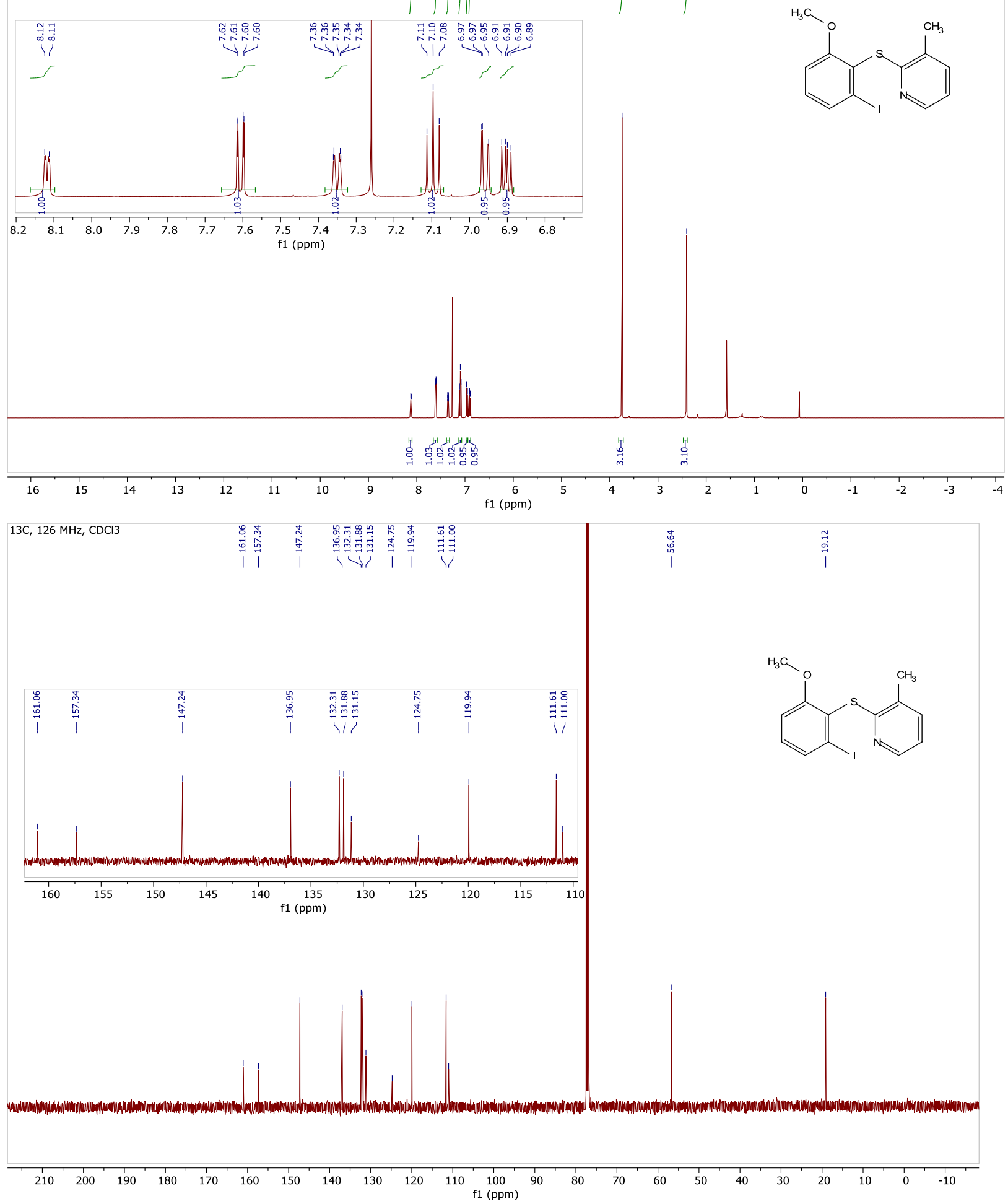

S22 
Figure S5.12: 2-(2-iodo-6-methyl-phenyl)sulfanyl)pyridine (5b):

$1 \mathrm{H}, 500 \mathrm{MHz}, \mathrm{CDCl} 3$
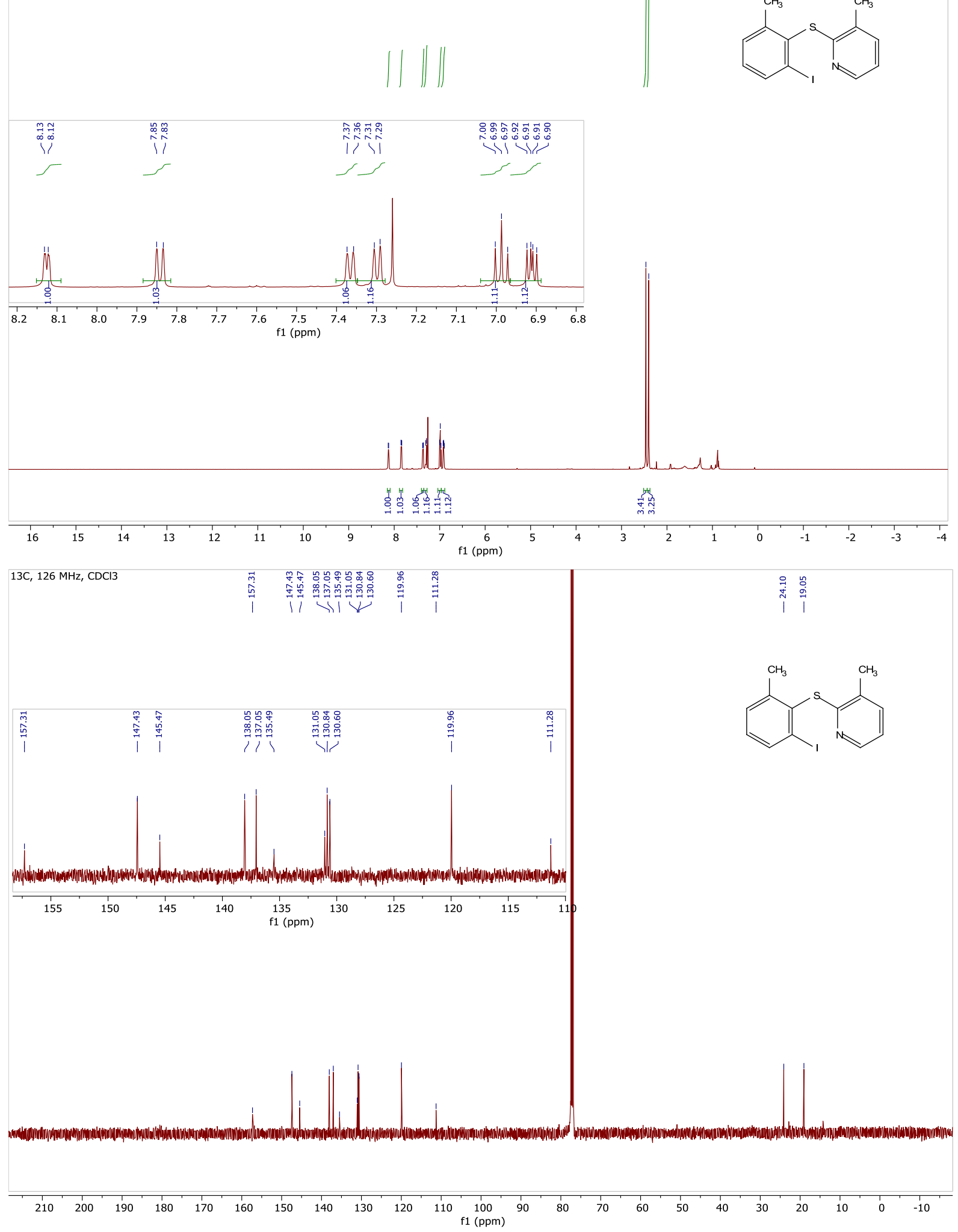

S23 
Figure S5.13: 2-((2,6-dichloro-phenyl)sulfanyl)-3-methyl-pyridine (1c):

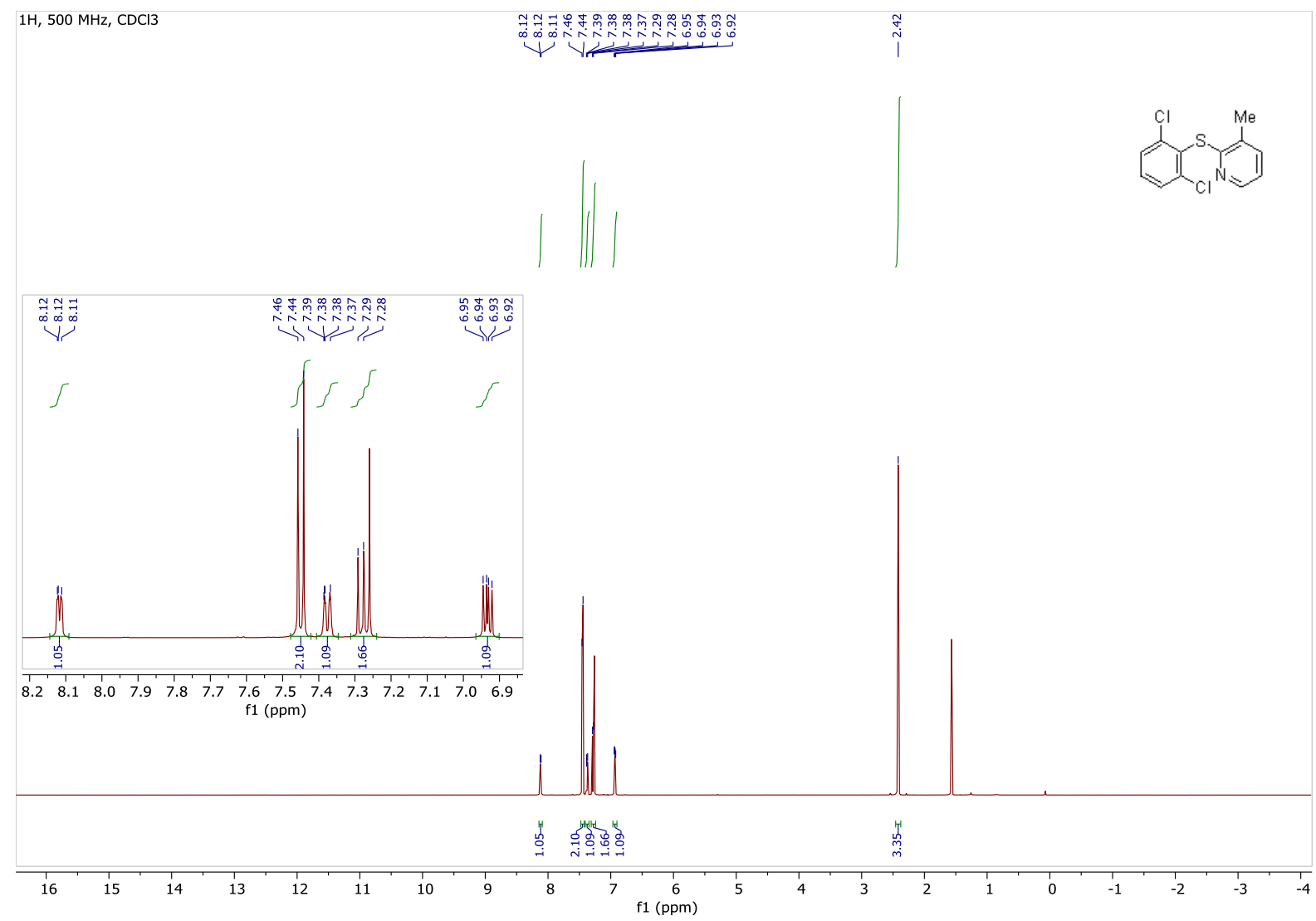

$13 \mathrm{C}, 126 \mathrm{MHz}, \mathrm{CDCl3}$
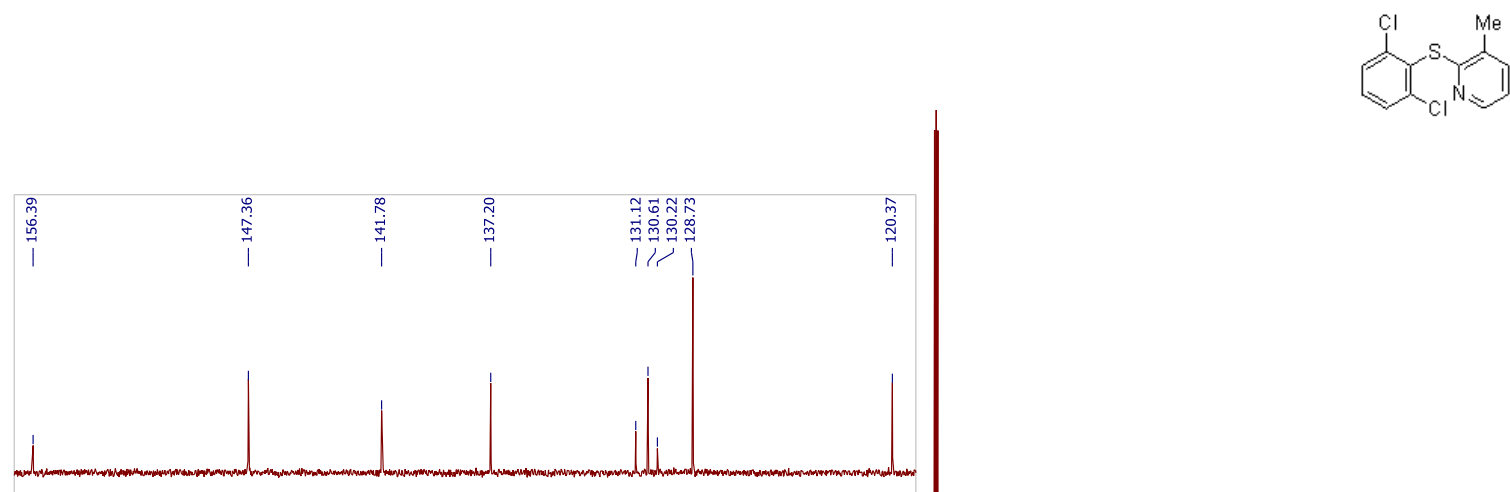

$\begin{array}{lllllllllllllllllllllllll}156 & 154 & 152 & 150 & 148 & 146 & 144 & 142 & 140 & 138 & 136 & 134 & 132 & 130 & 128 & 126 & 124 & 122 & 120\end{array}$

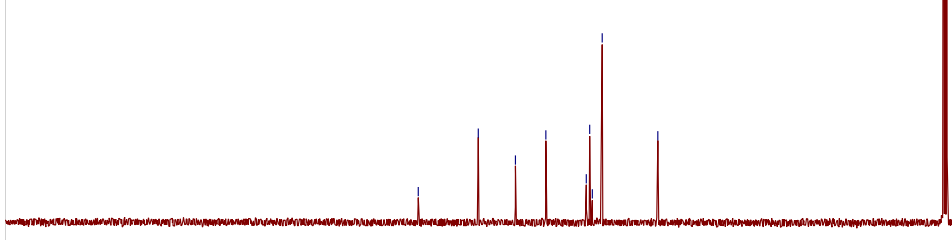

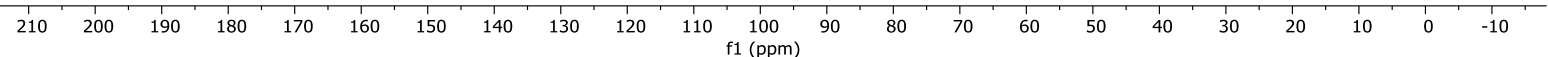


Figure S5.14: 2-((2-chloro-6-trifluoro-phenyl)sulfanyl)-3-methyl-pyridine (3c):
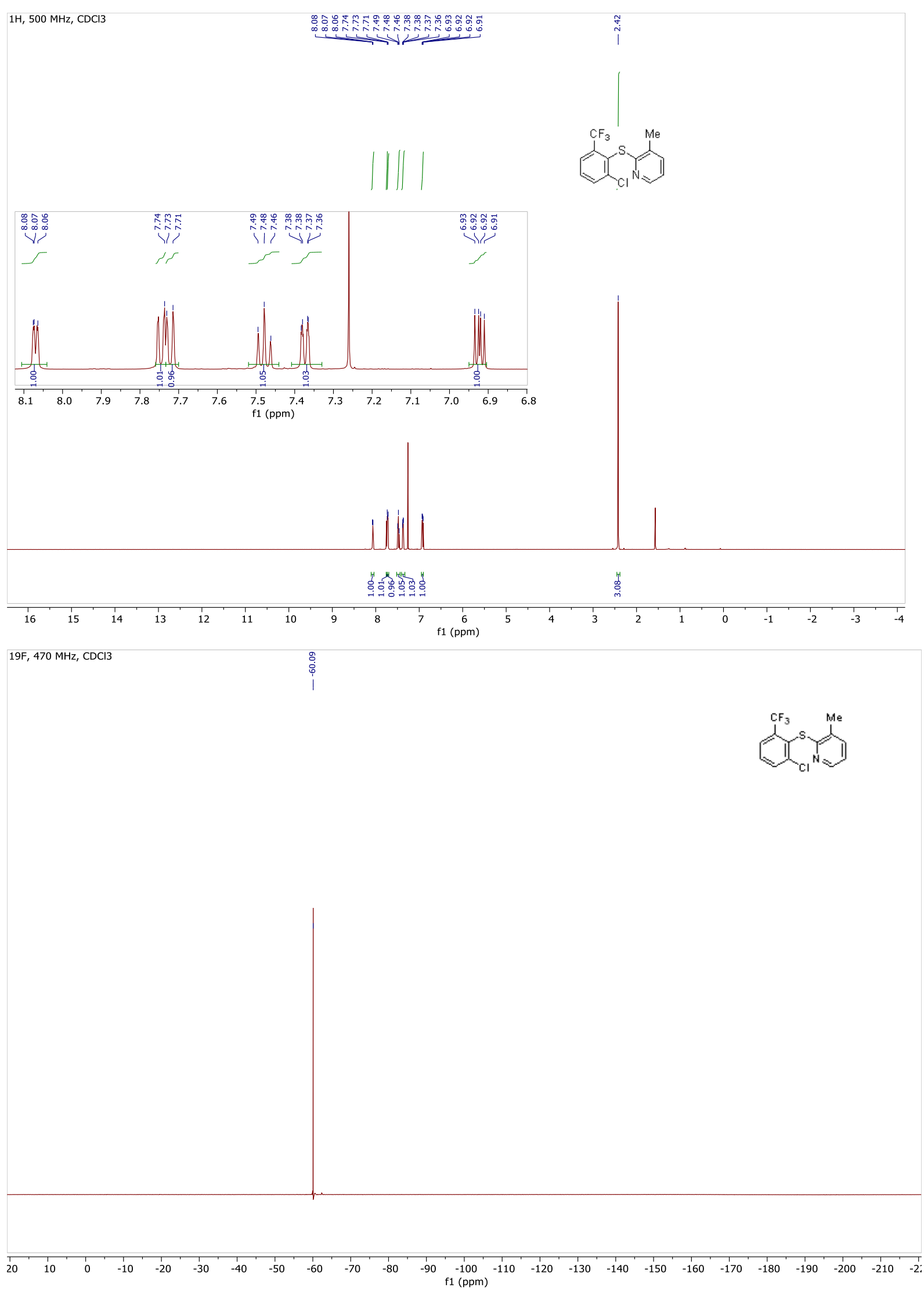

S25 


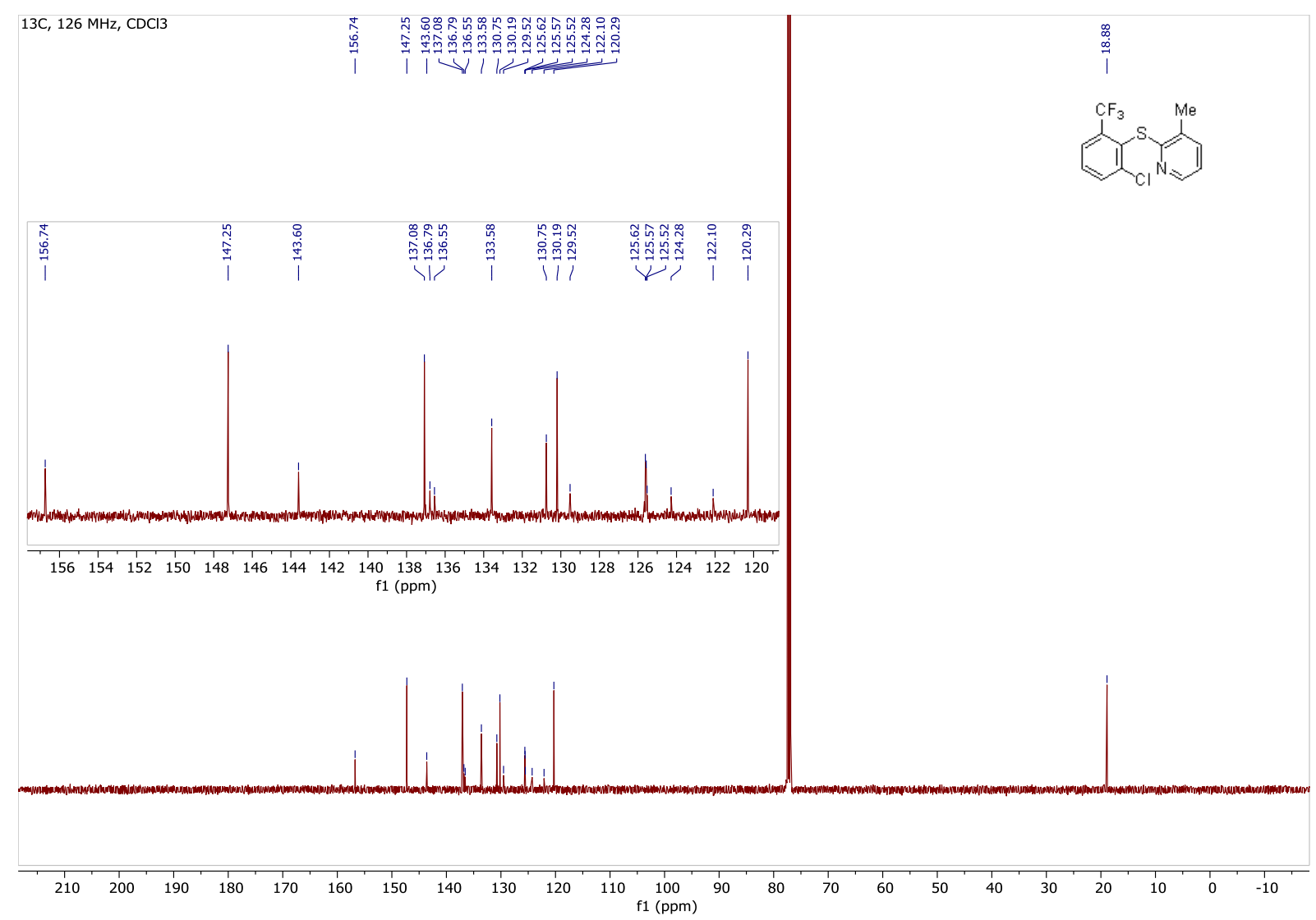


Figure S5.15: 2-((2-chloro-6-methyl-phenyl)sulfanyl)-3-methyl-pyridine (5c):
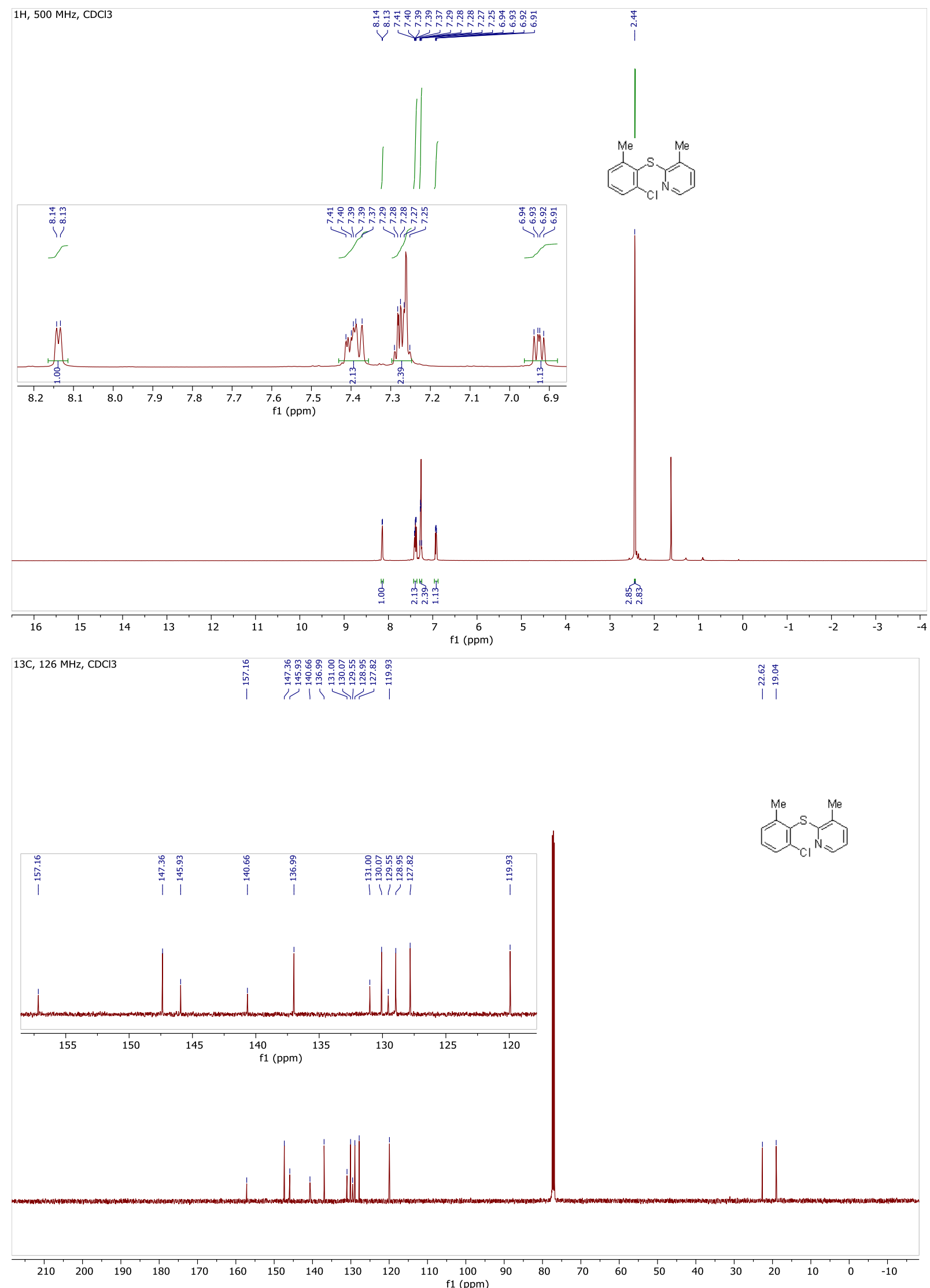
Figure S5.16: 2-(2,6-dibromo-4-tert-butyle-benzensulfanyl)-3-methyl-pyridine (9d):
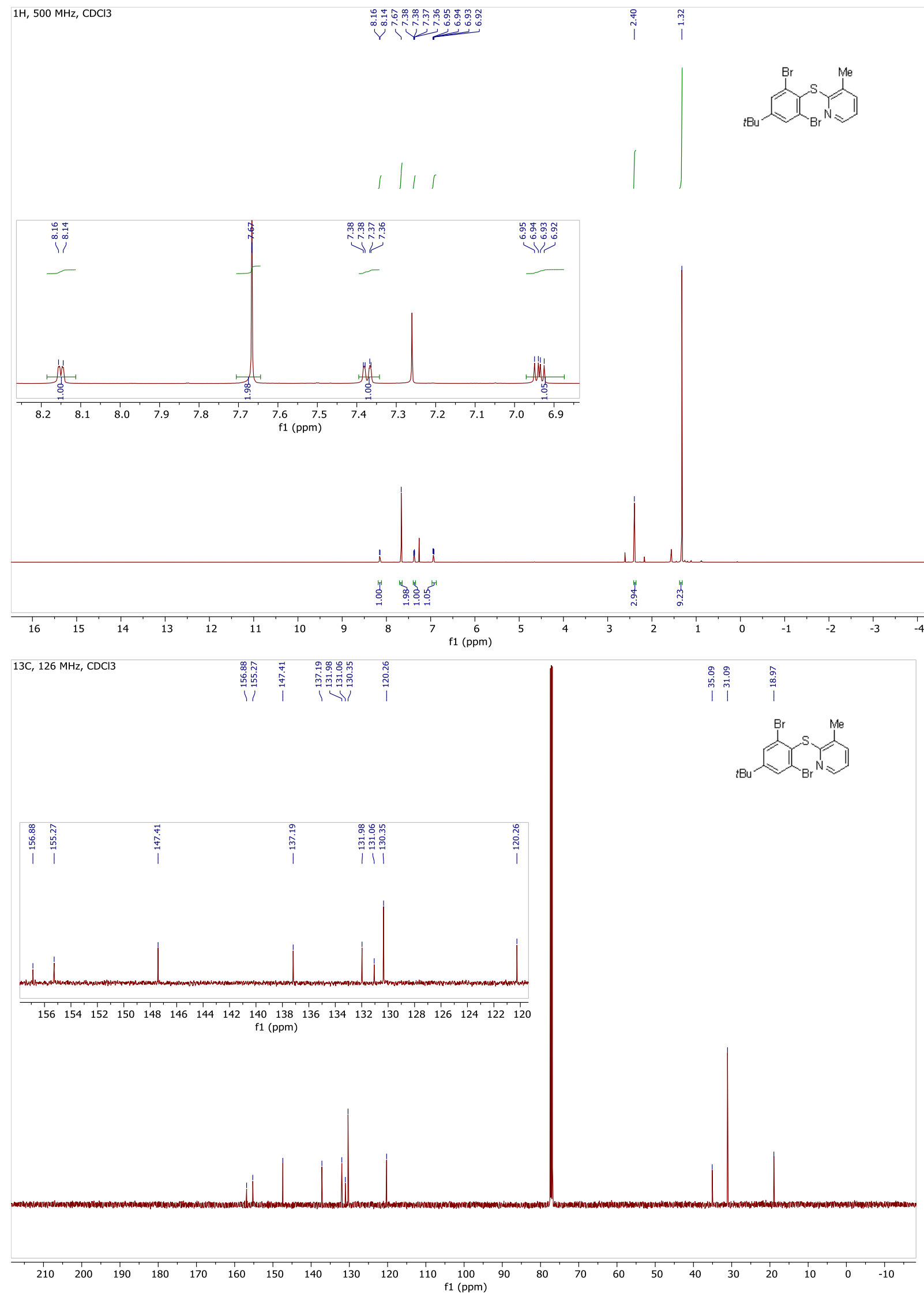
Figure S5.17: 2-(2,6-dibromo-4-chloro-benzensulfanyl)-3-methyl-pyridine (15d):

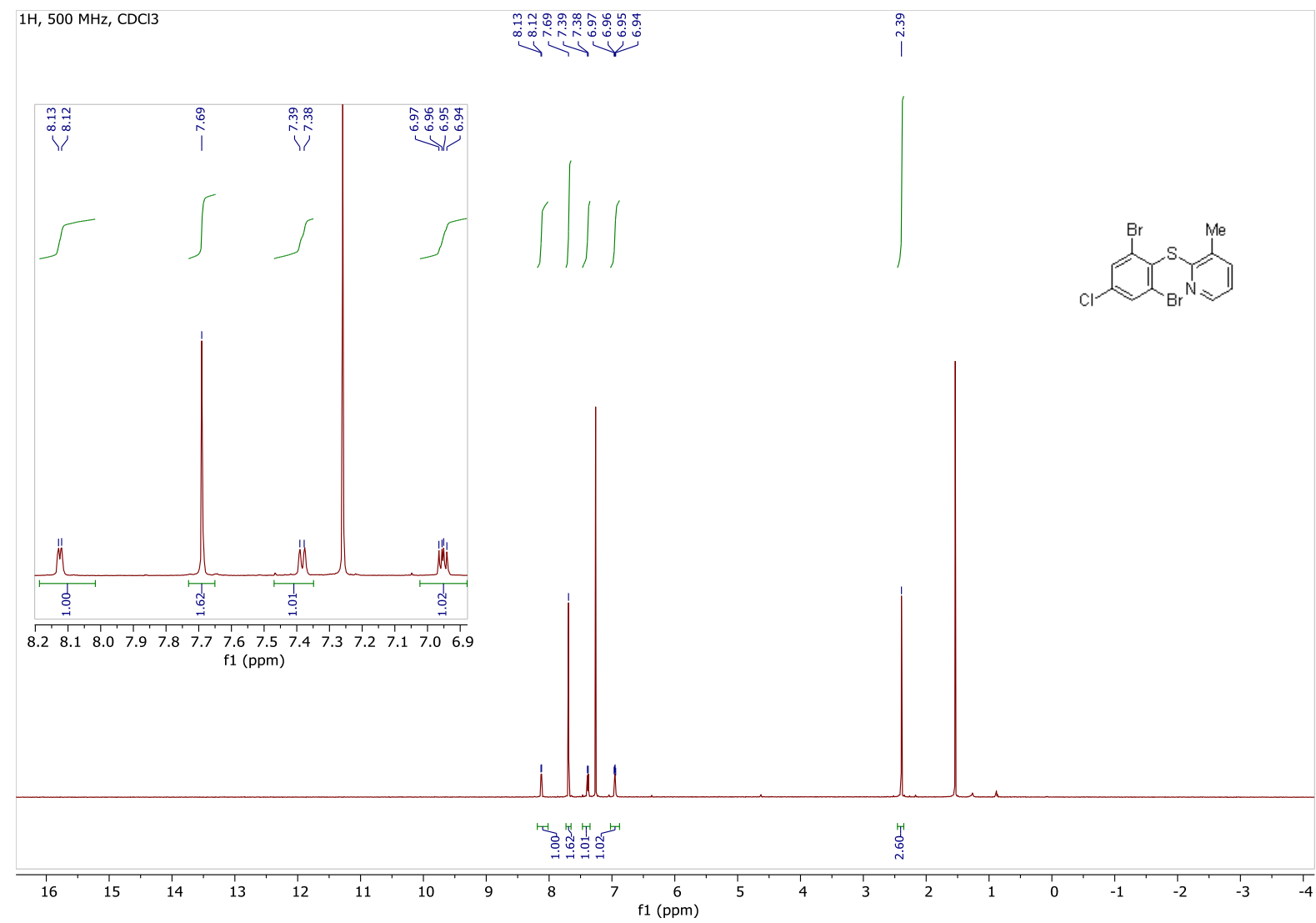

$13 \mathrm{C}, 126 \mathrm{MHz}, \mathrm{CDCl} 3$
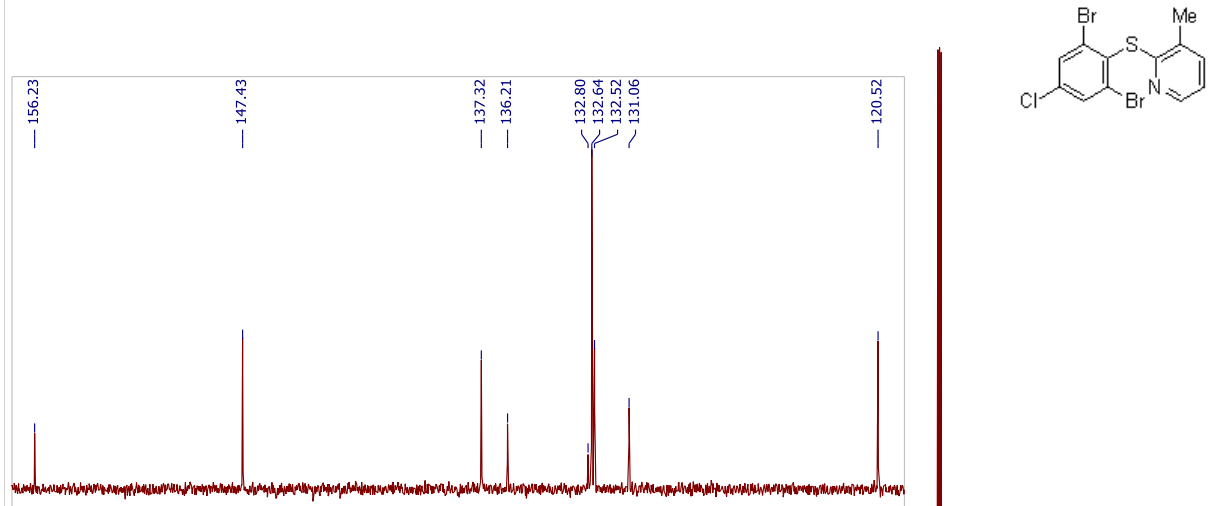

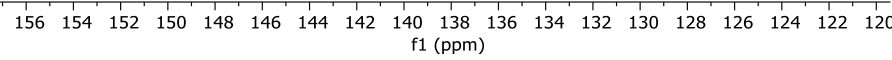

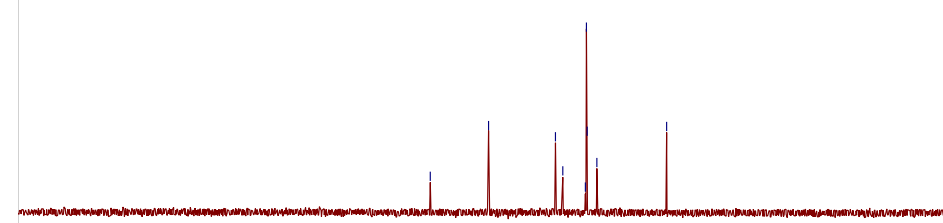

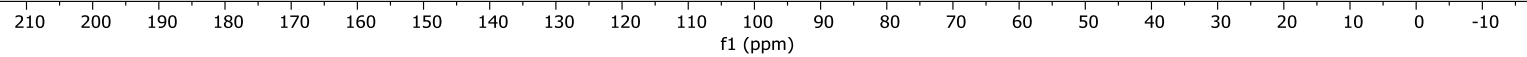


Figure S5.18: 2-(2,6-dibromo-benzensulfanyl)-3-methyl-pyridine (11d):

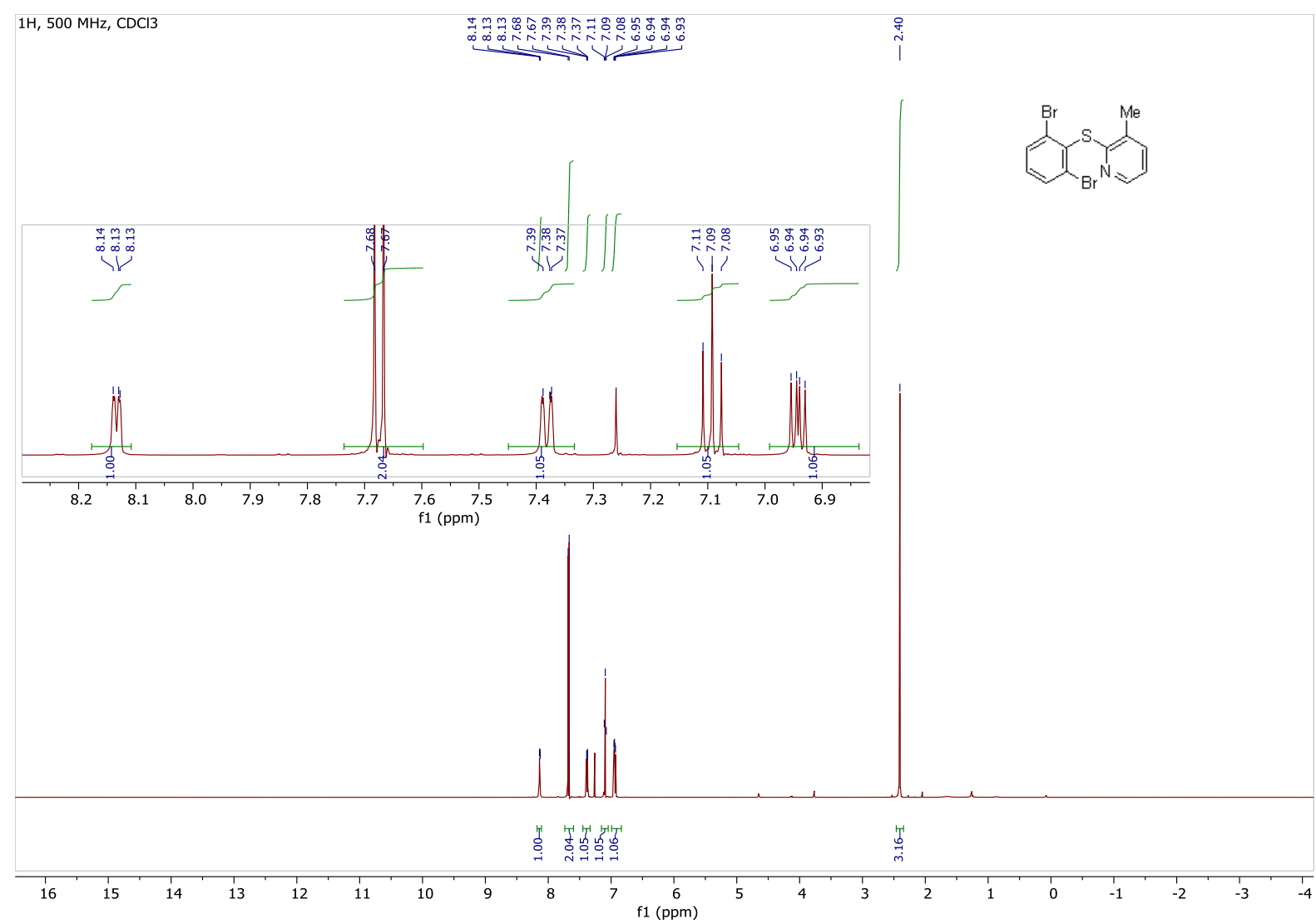

$13 \mathrm{C}, 126 \mathrm{MHz}, \mathrm{CDCl} 3$

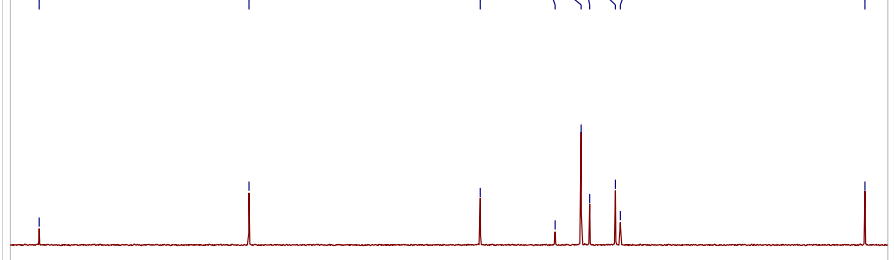

$\begin{array}{lllllllllllllllllll}156 & 154 & 152 & 150 & 148 & 146 & 144 & 142 & 140 & 138 & 136 & 134 & 132 & 130 & 128 & 126 & 124 & 122 & 120\end{array}$ f1 (ppm)

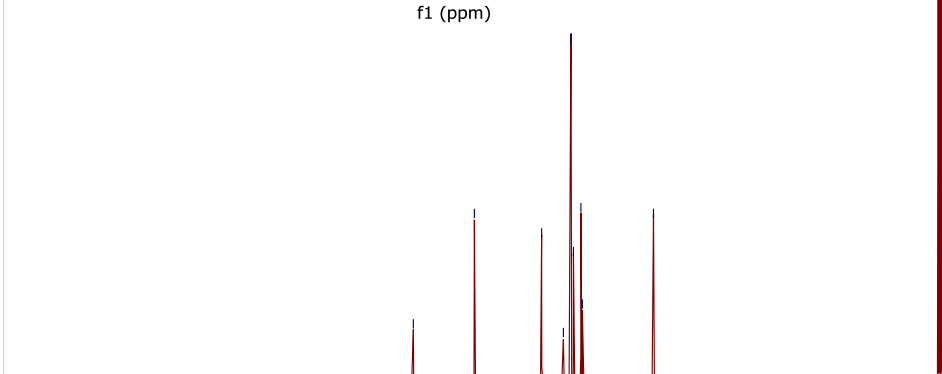

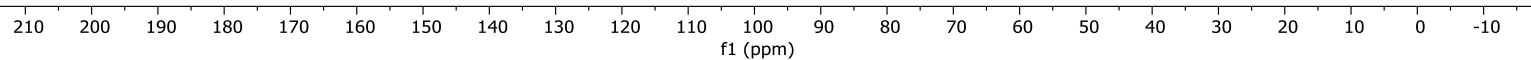


Figure S5.19: 2-(2,6-dibromo-3-methyl-benzensulfanyl)-3-methyl-pyridine (12d):
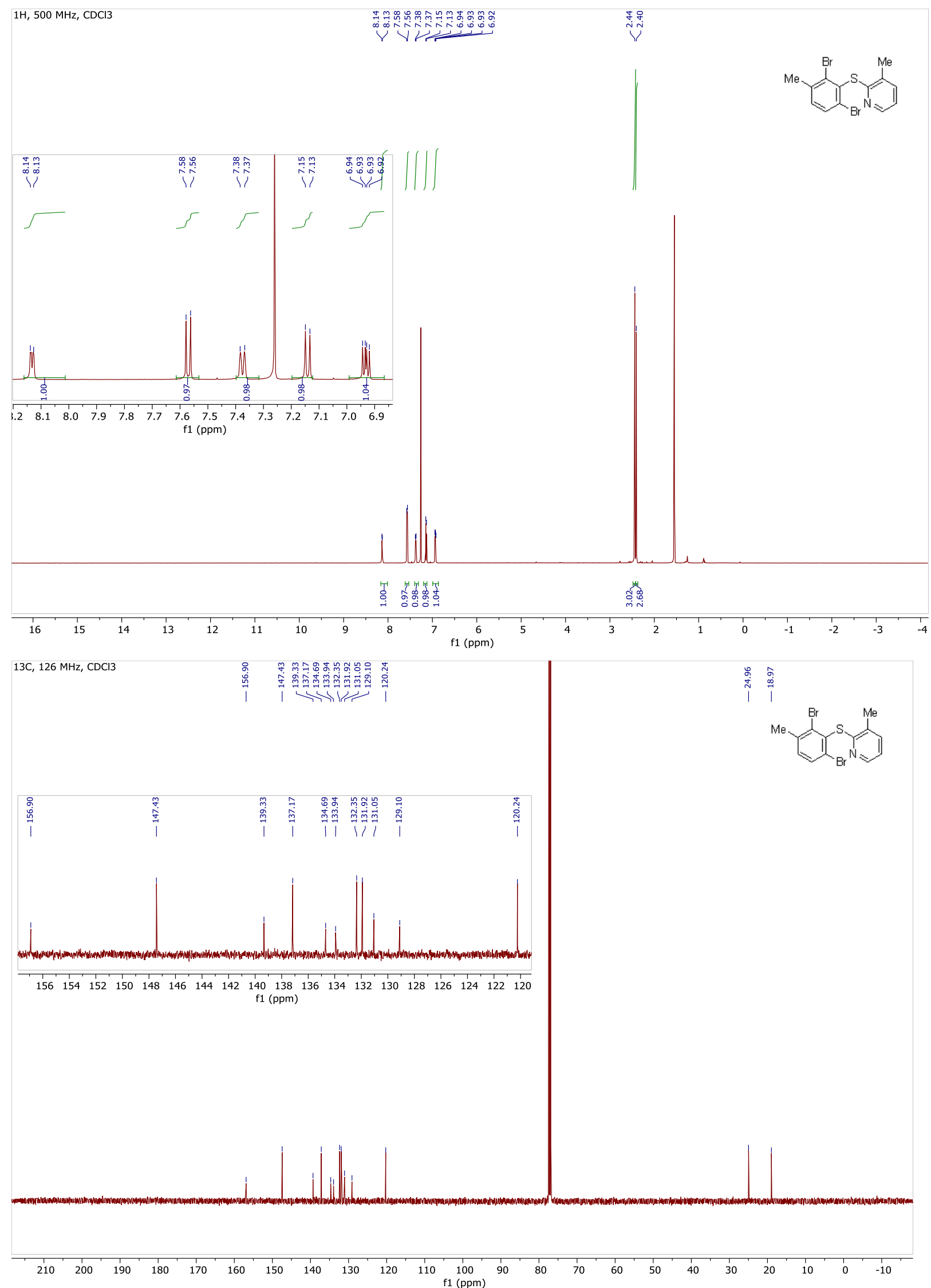
Figure S5.20: 2-(2,6-dibromo-3-chloro-benzensulfanyl)-3-methyl-pyridine (13d):
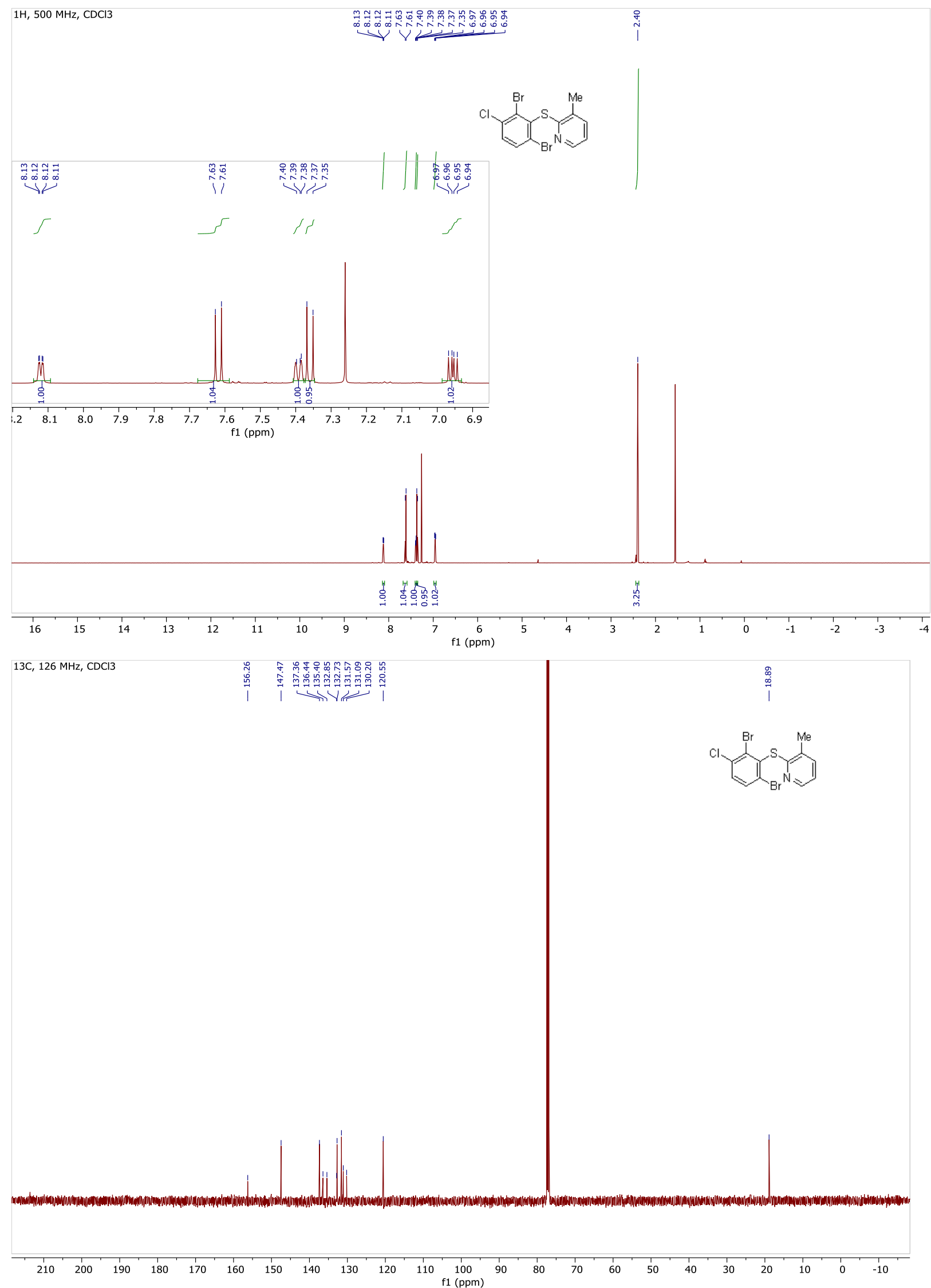
Figure S5.21: 2-(2,6-dibromobenzensulfanyl)pyridine (14d):

$1 \mathrm{H} \mathrm{NMR,}, 500 \mathrm{MHz}, \mathrm{CDCl}$
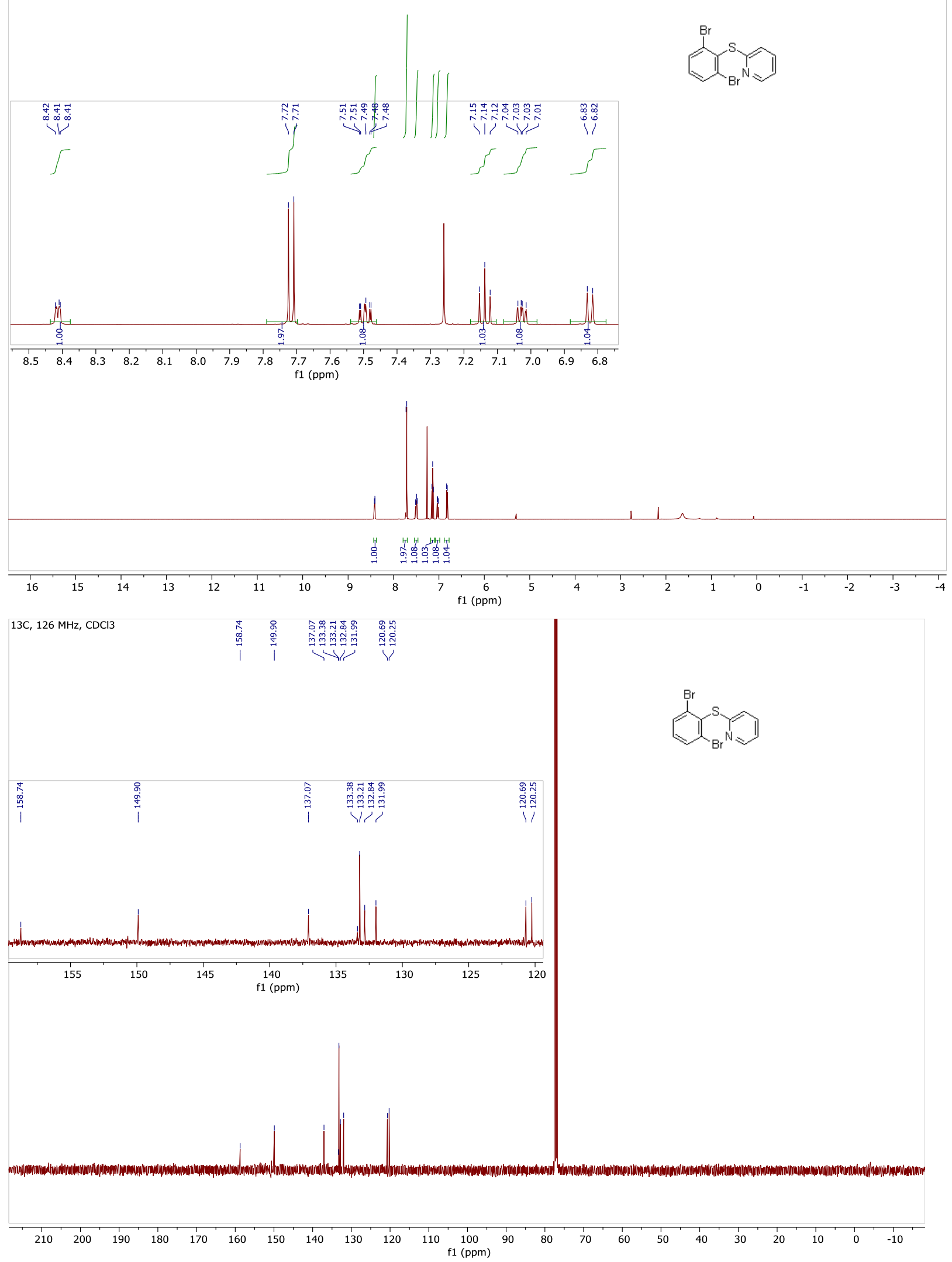

S33 
Figure S5.22: 2-((2,6-dibromo-4-chloro-phenyl)sulfanyl)pyridine (15d):
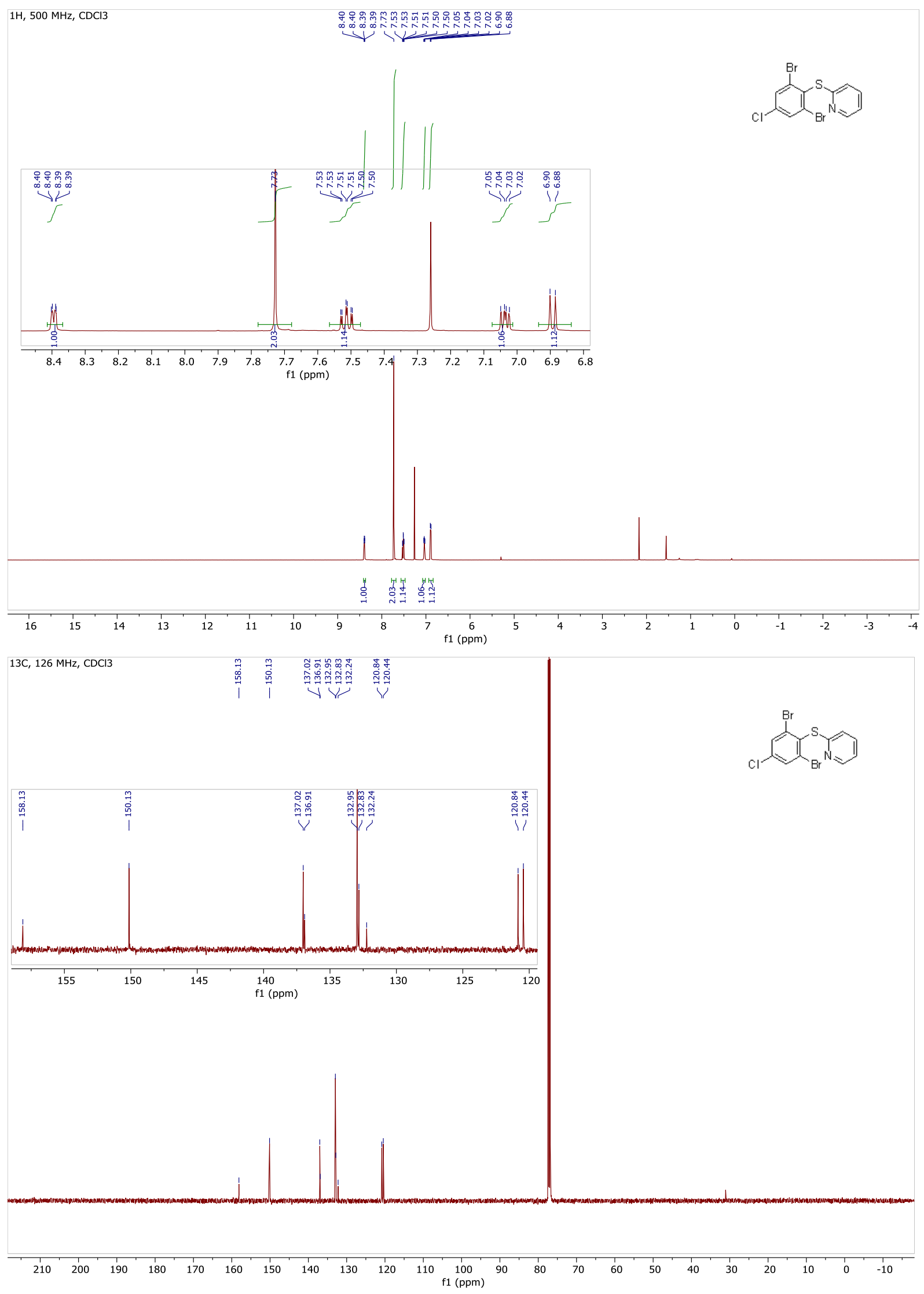

S34 
Figure S5.23: 2-((2,6-diiodo-4-tert-butyl-phenyl)sulfanyl)-3-methyl-pyridine (9e):
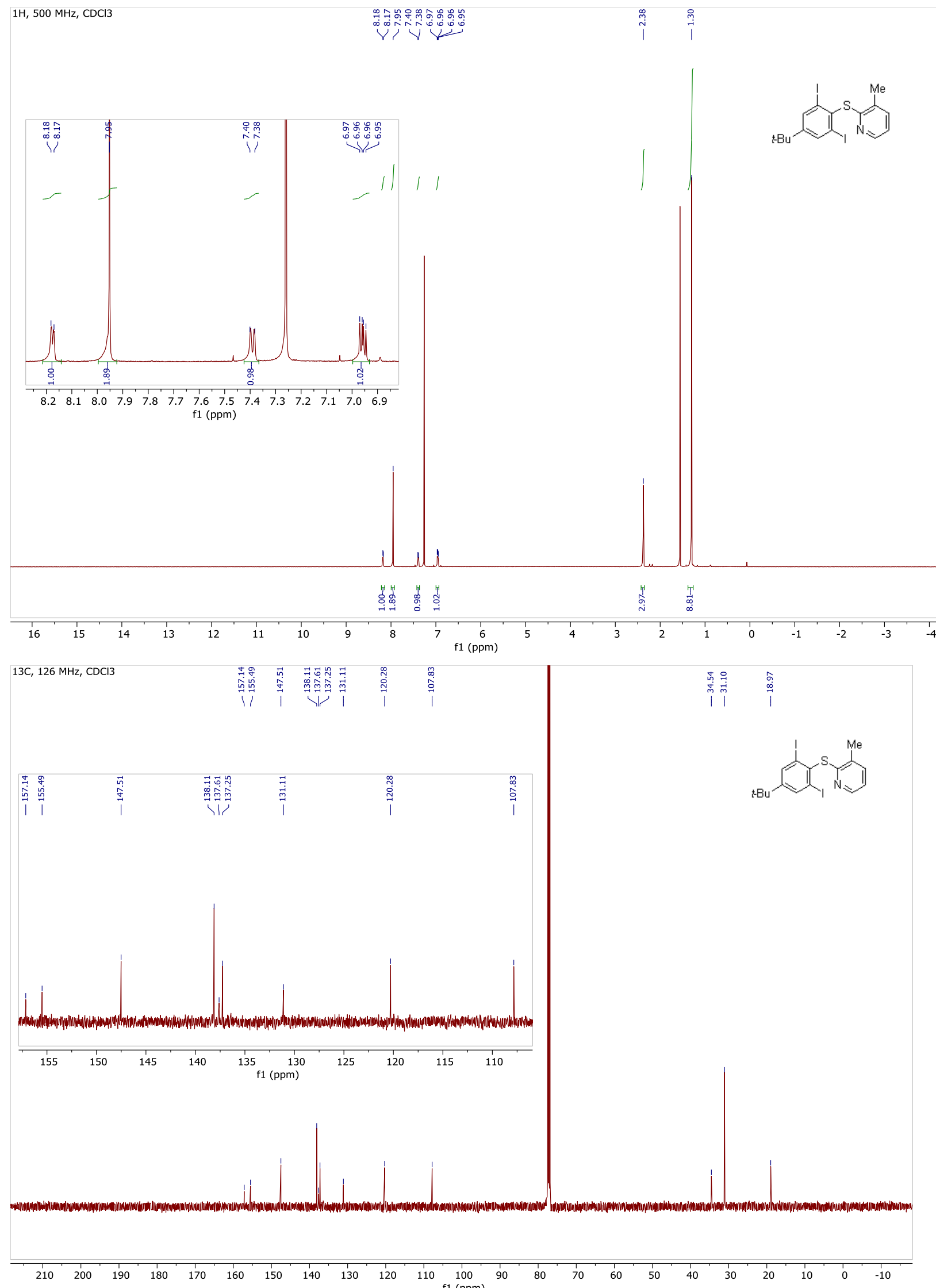
Figure S5.24: 2-((2,6-diiodo-4-chloro-phenyl)sulfanyl)-3-methyl-pyridine (10e):
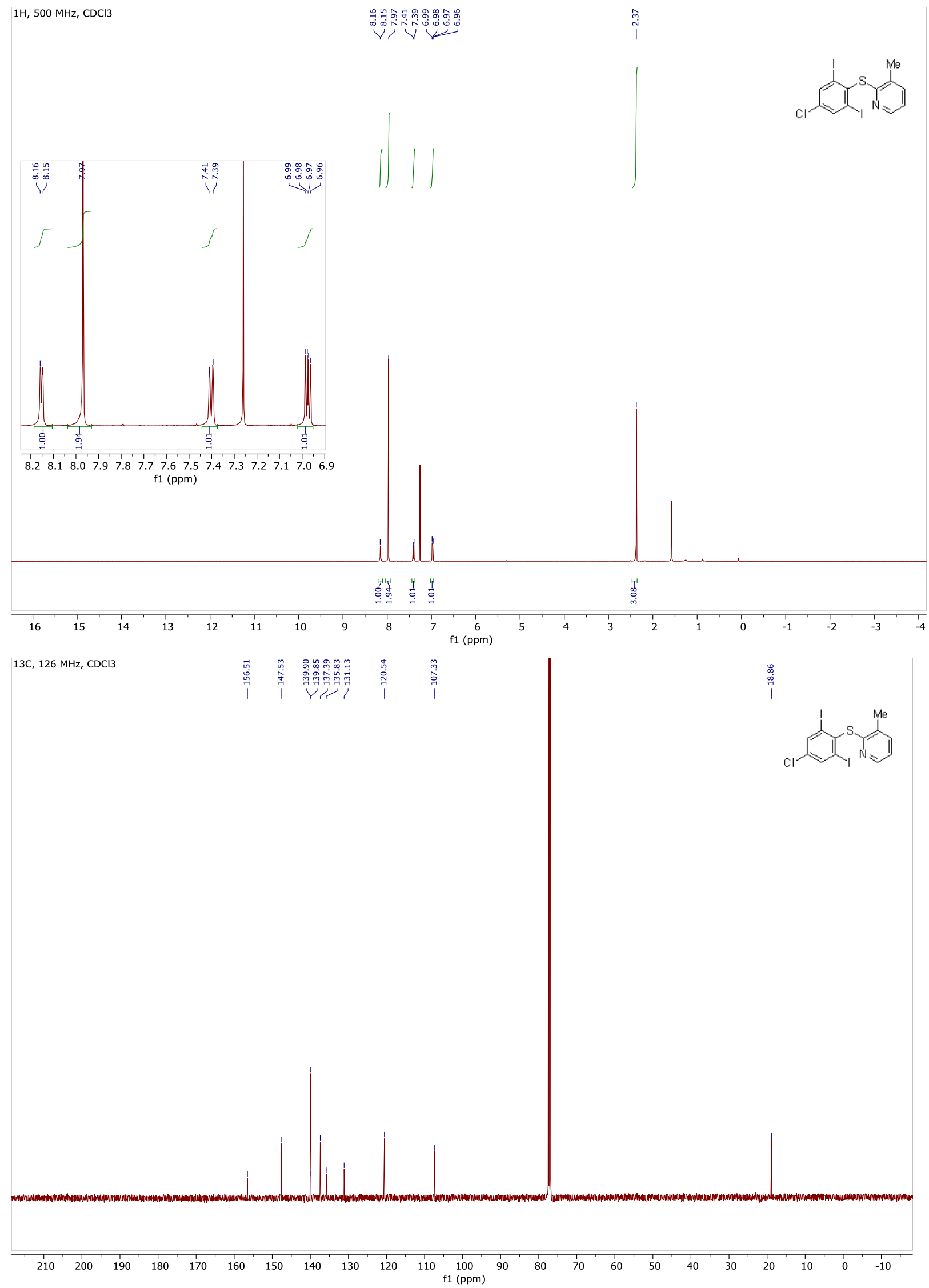
Figure S5.25: 2-((2,6-diiodo-phenyl)sulfanyl)-3-methyl-pyridine (11e):
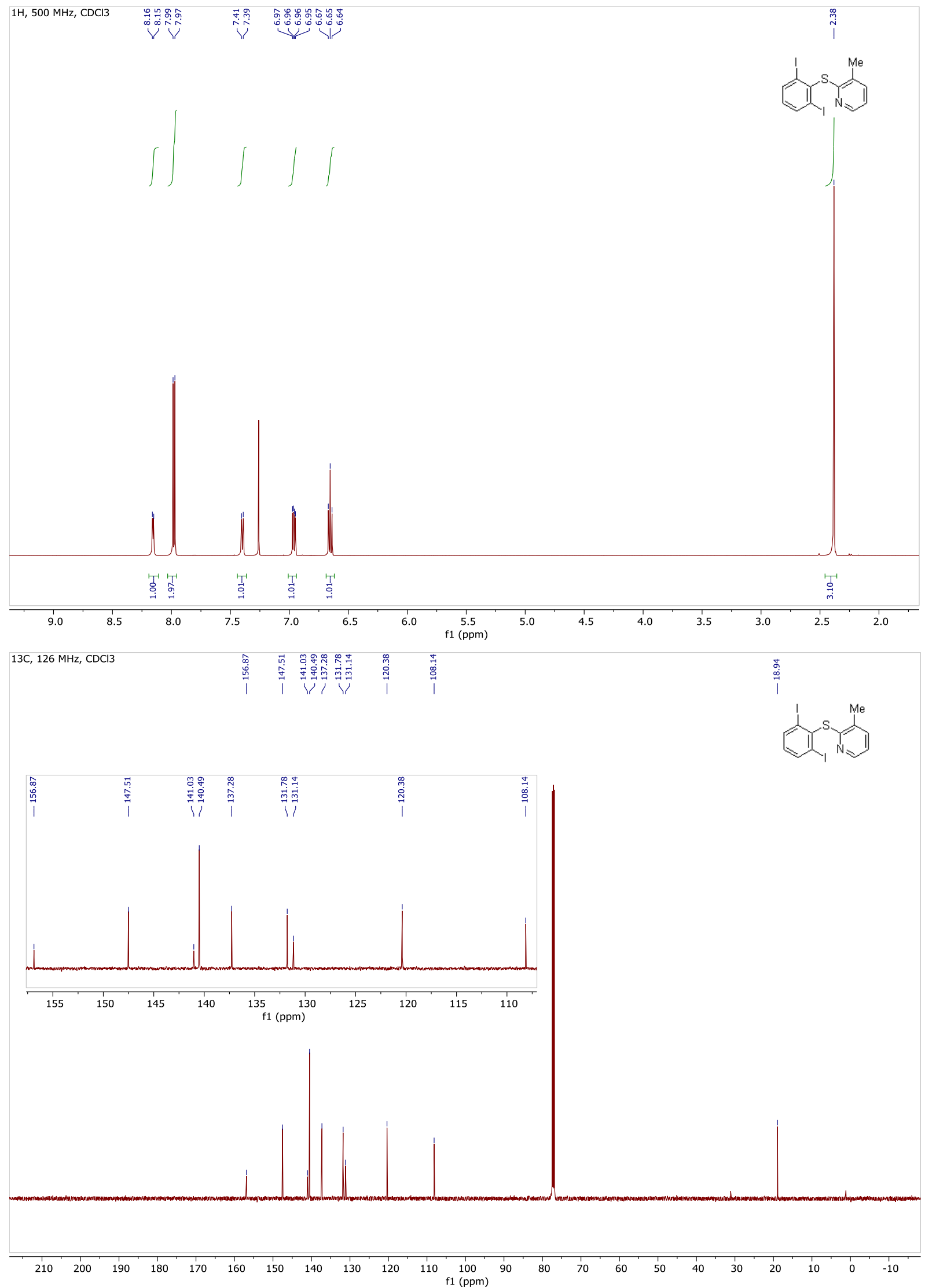
Figure S5.26: 2-((2,6-diiodo-3-methyl-phenyl)sulfanyl)-3-methyl-pyridine (12e):
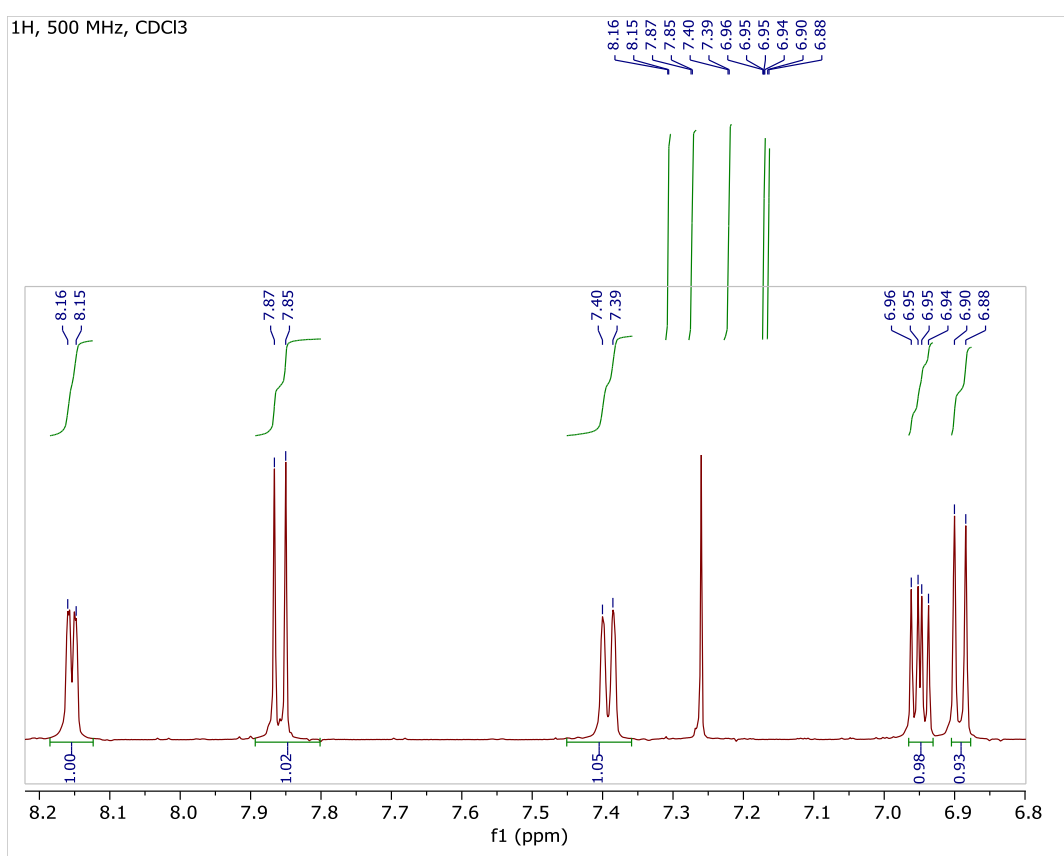

$\stackrel{\vec{n}}{\stackrel{\infty}{\stackrel{\infty}{\sim}} / 1}$

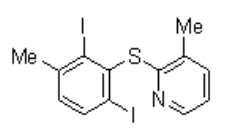

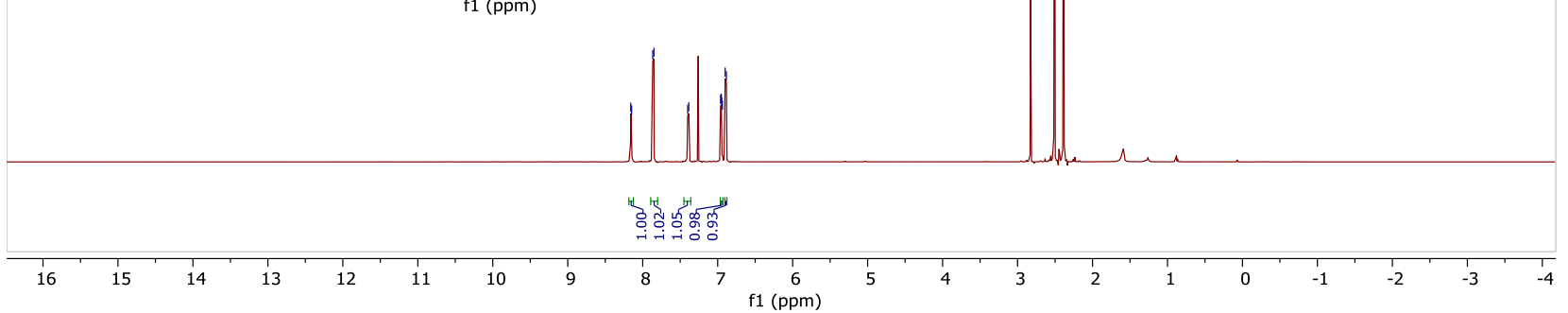
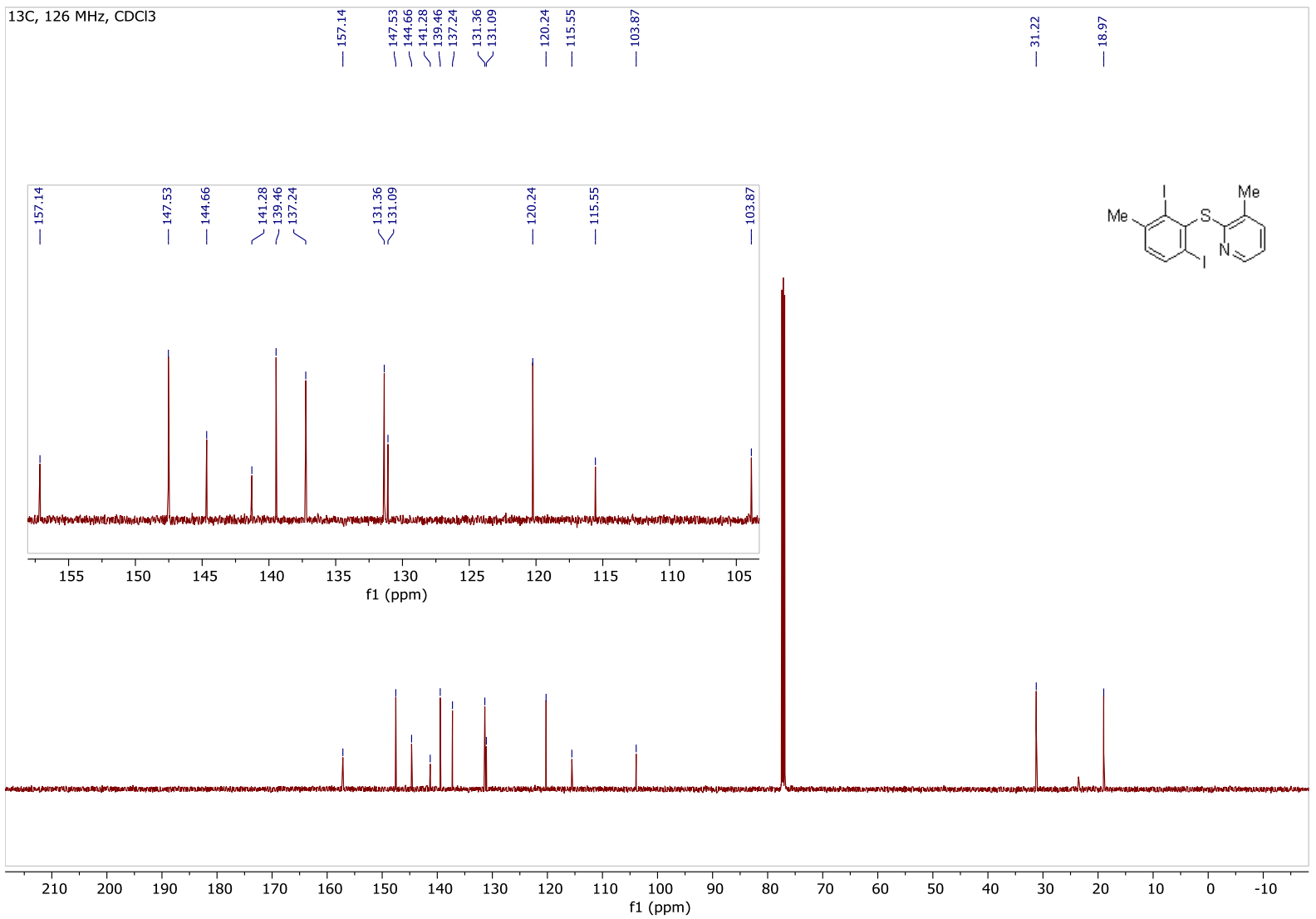

S38 
Figure S5.27: 2-((2,6-diiodo-3-chloro-phenyl)sulfanyl)-3-methyl-pyridine (13e):
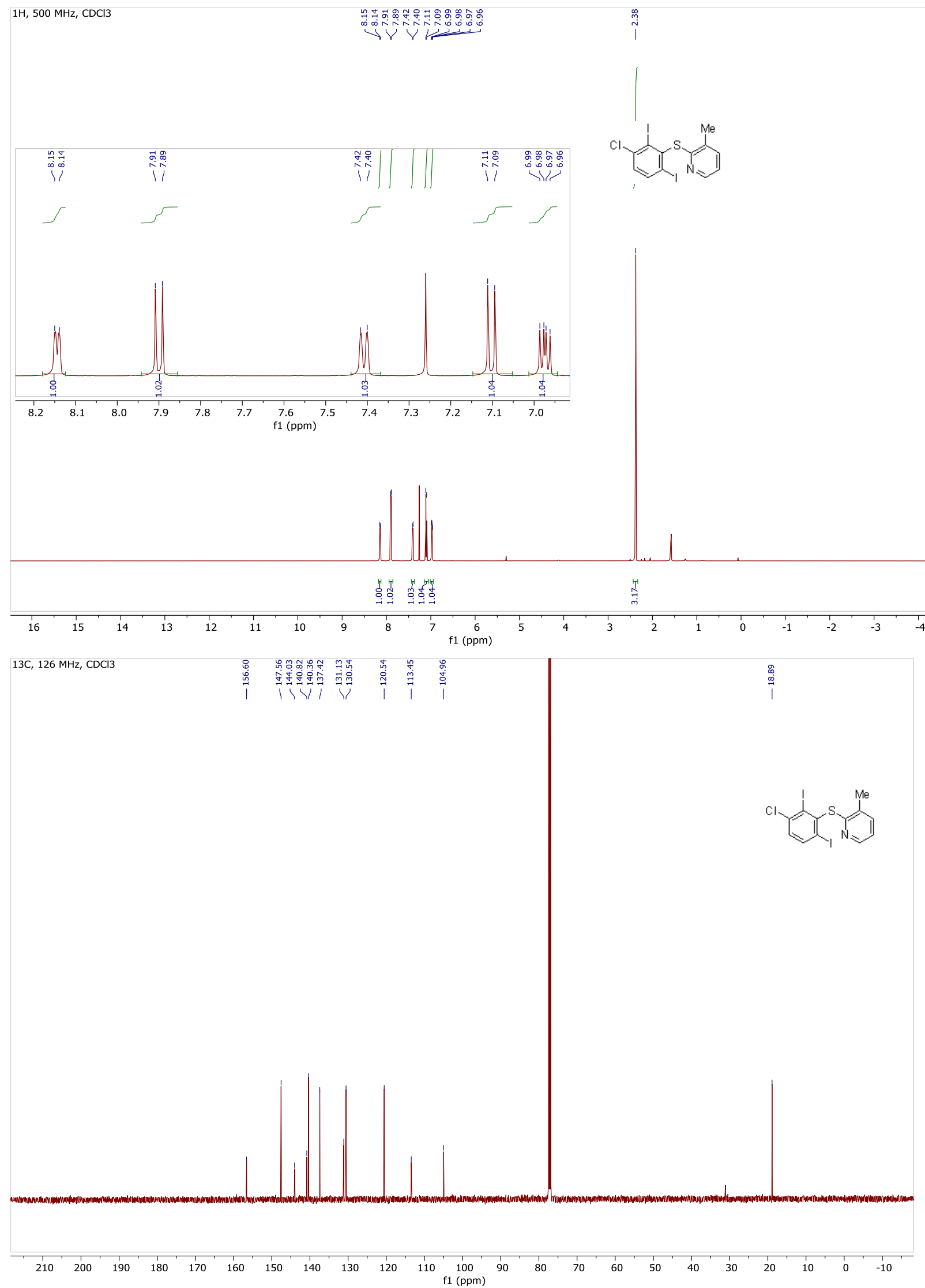
Figure S5.28: 2-(2-diacetoxy-6-methyl-phenylsulfanyl)-3-methyl-pyridine (5f):

$1 \mathrm{H}, 500 \mathrm{MHz}, \mathrm{CDCl} 3$

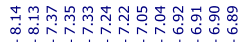

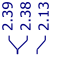

$\underset{\substack{+\infty \\ \infty}}{\substack{\infty \\ \infty}}$
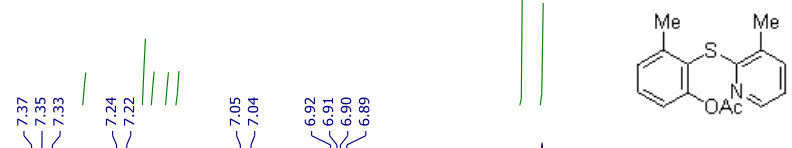

रा it it Vili
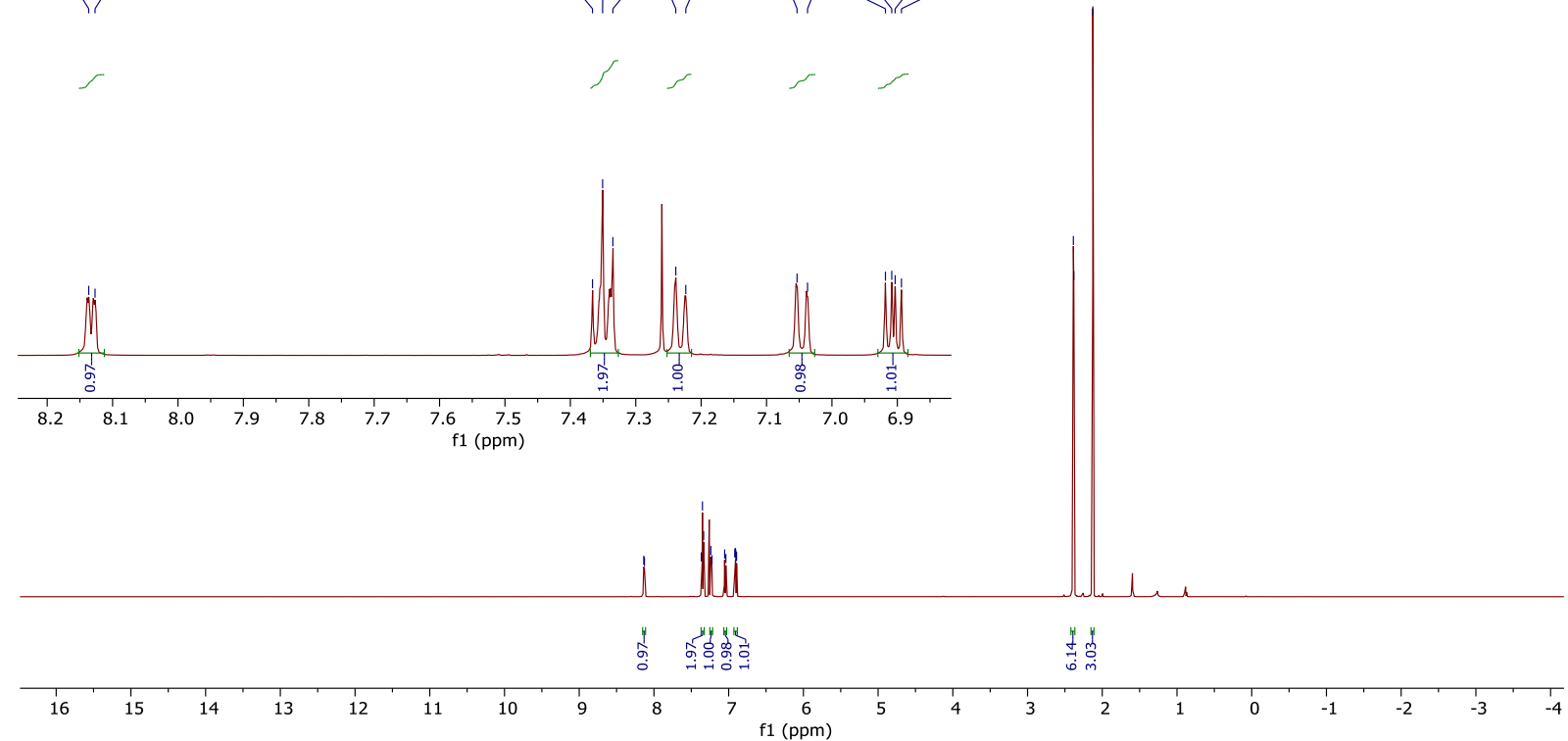

$13 \mathrm{C}, 126 \mathrm{MHz}, \mathrm{CDCl} 3$

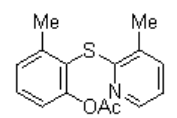

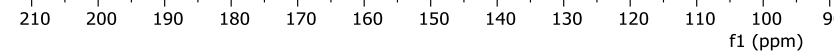


Figure S5.29: 2-(2-diacetoxy-6-methyl-phenylsulfanyl)-pyridine (16f):
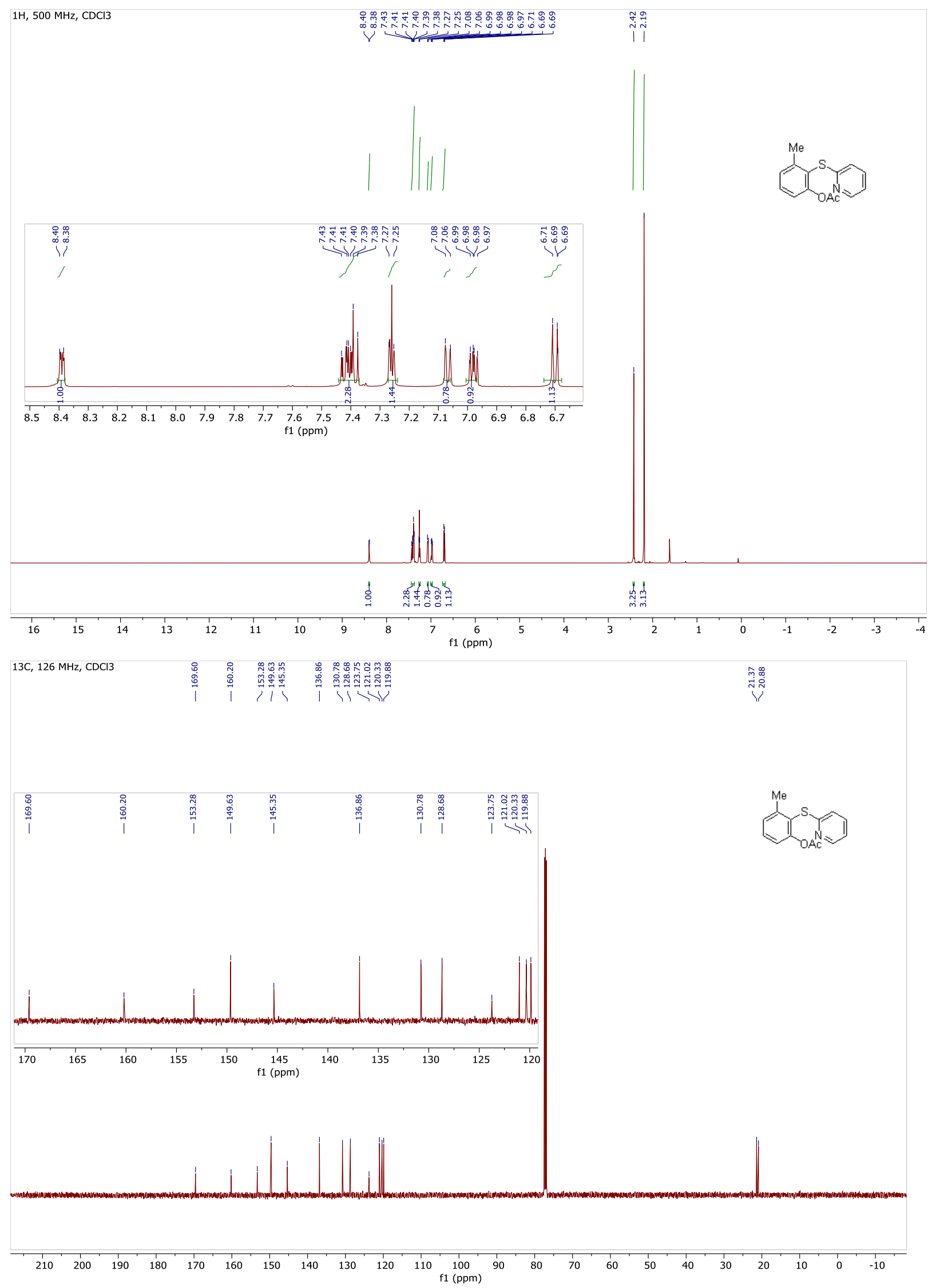
Figure S5.30: 2-(2-acetoxy-phenylsulfanyl)-3-methyl-pyridine (17f):

$1 \mathrm{H}, 500 \mathrm{MHz}, \mathrm{CDCl} 3$

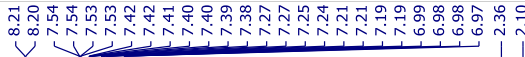

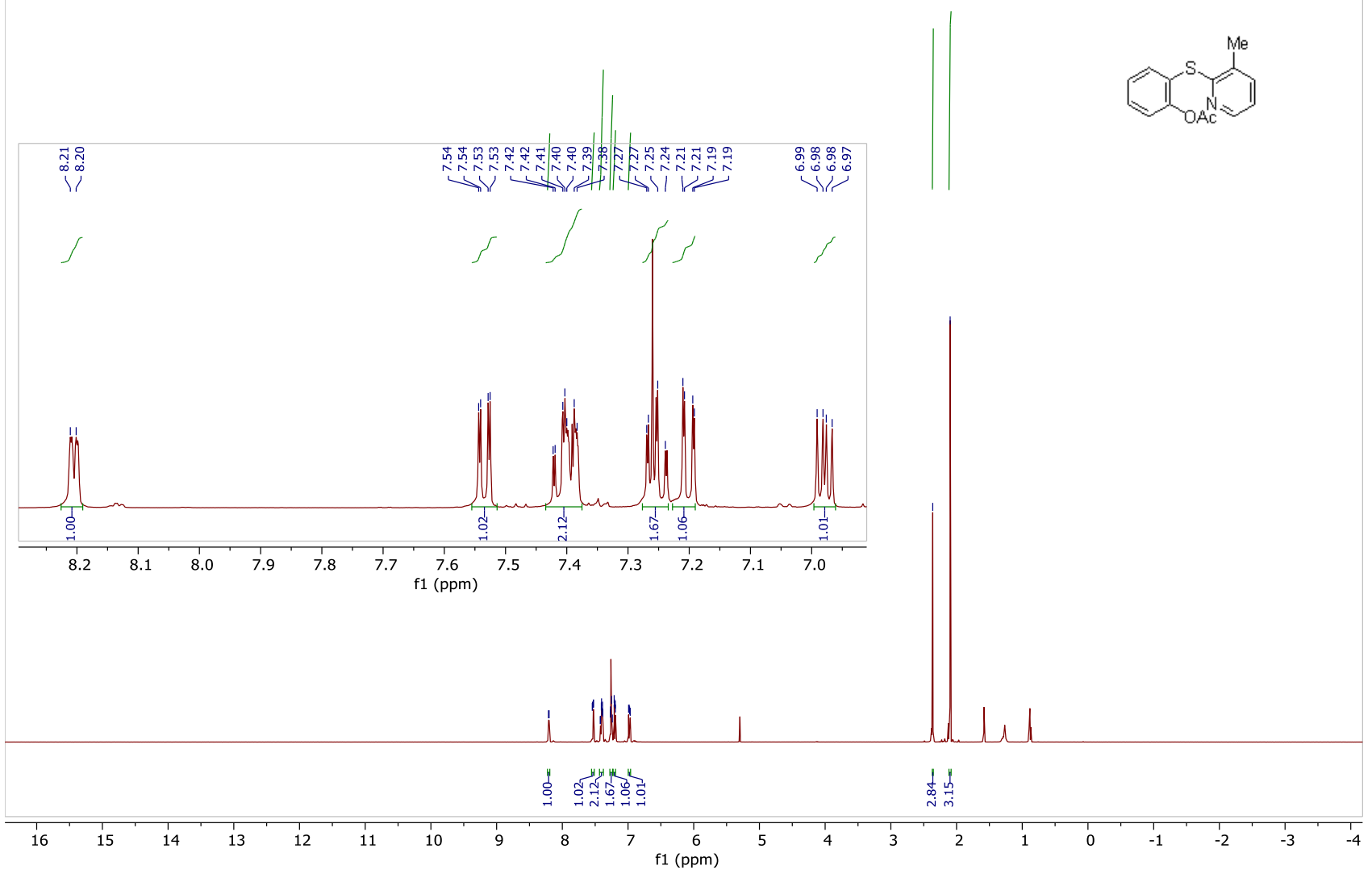

$13 \mathrm{C}, 126 \mathrm{MHz}, \mathrm{CDCl} 3$

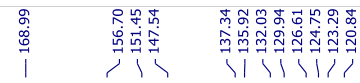

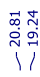
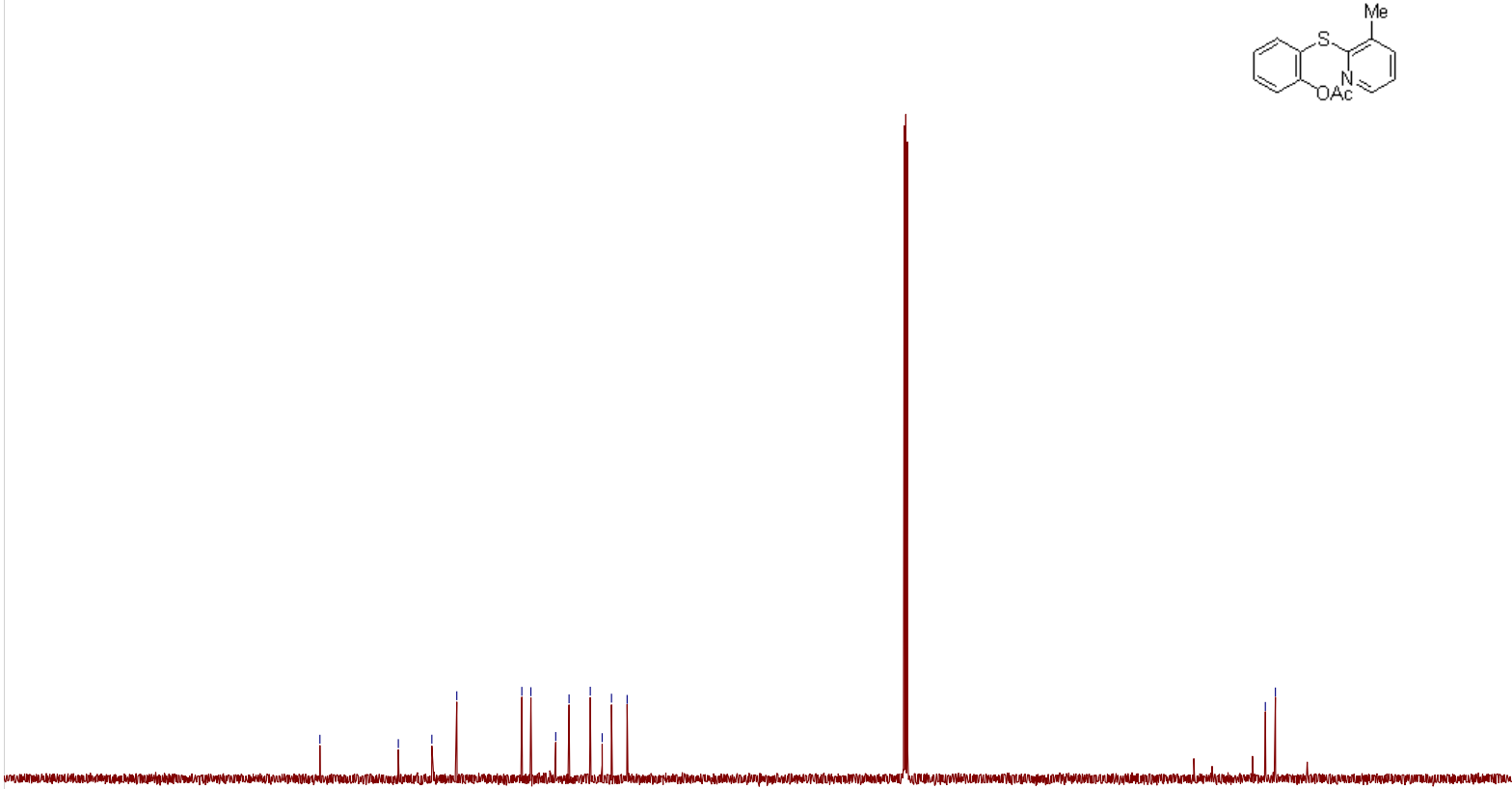

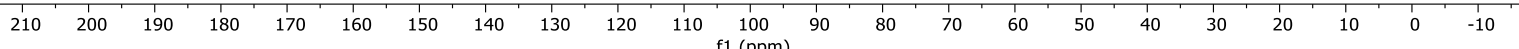


Figure S5.31: 2-(2,6-diacetoxy-phenylsulfanyl)-3-methyl-pyridine (17g):

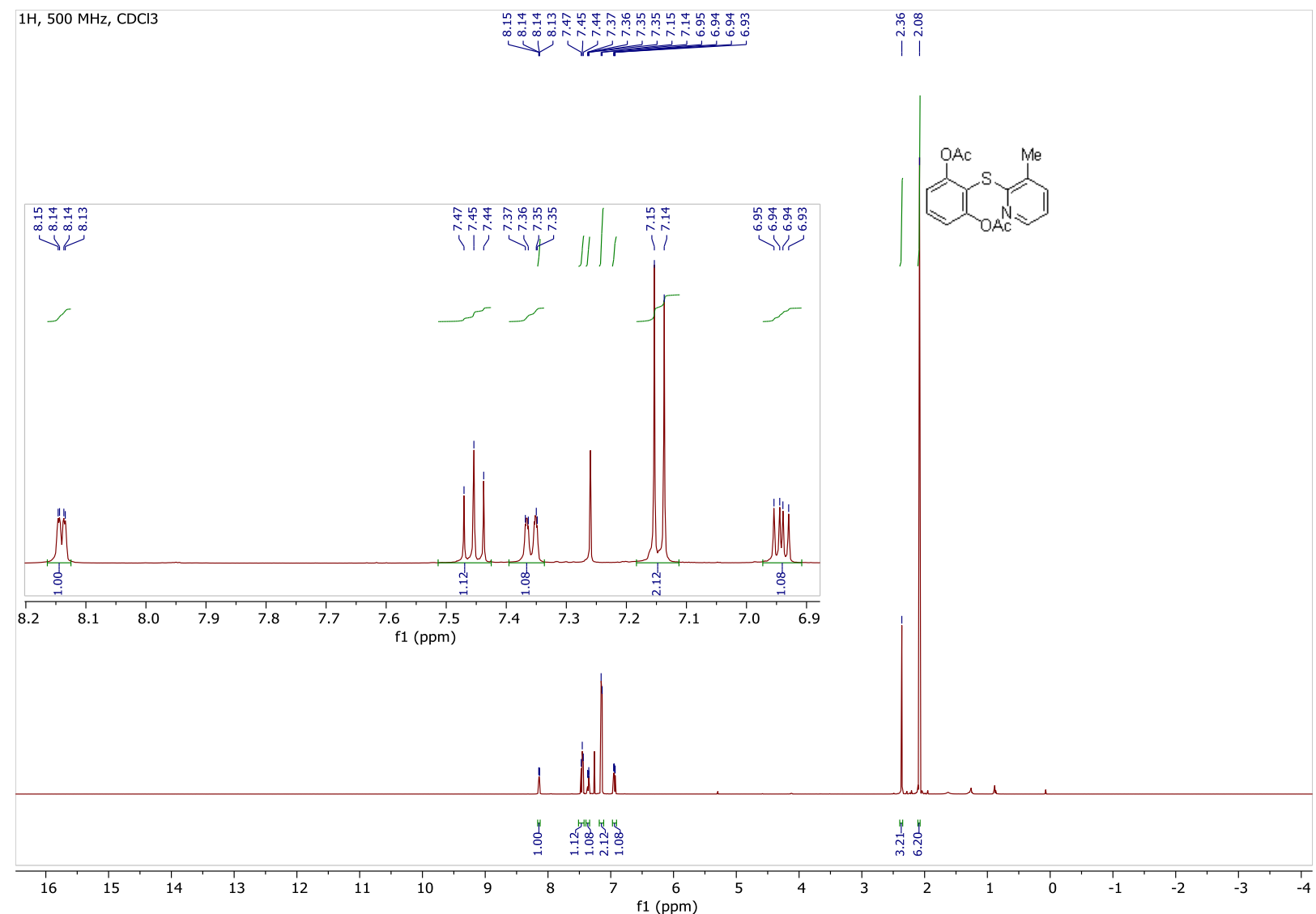

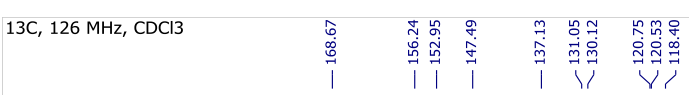

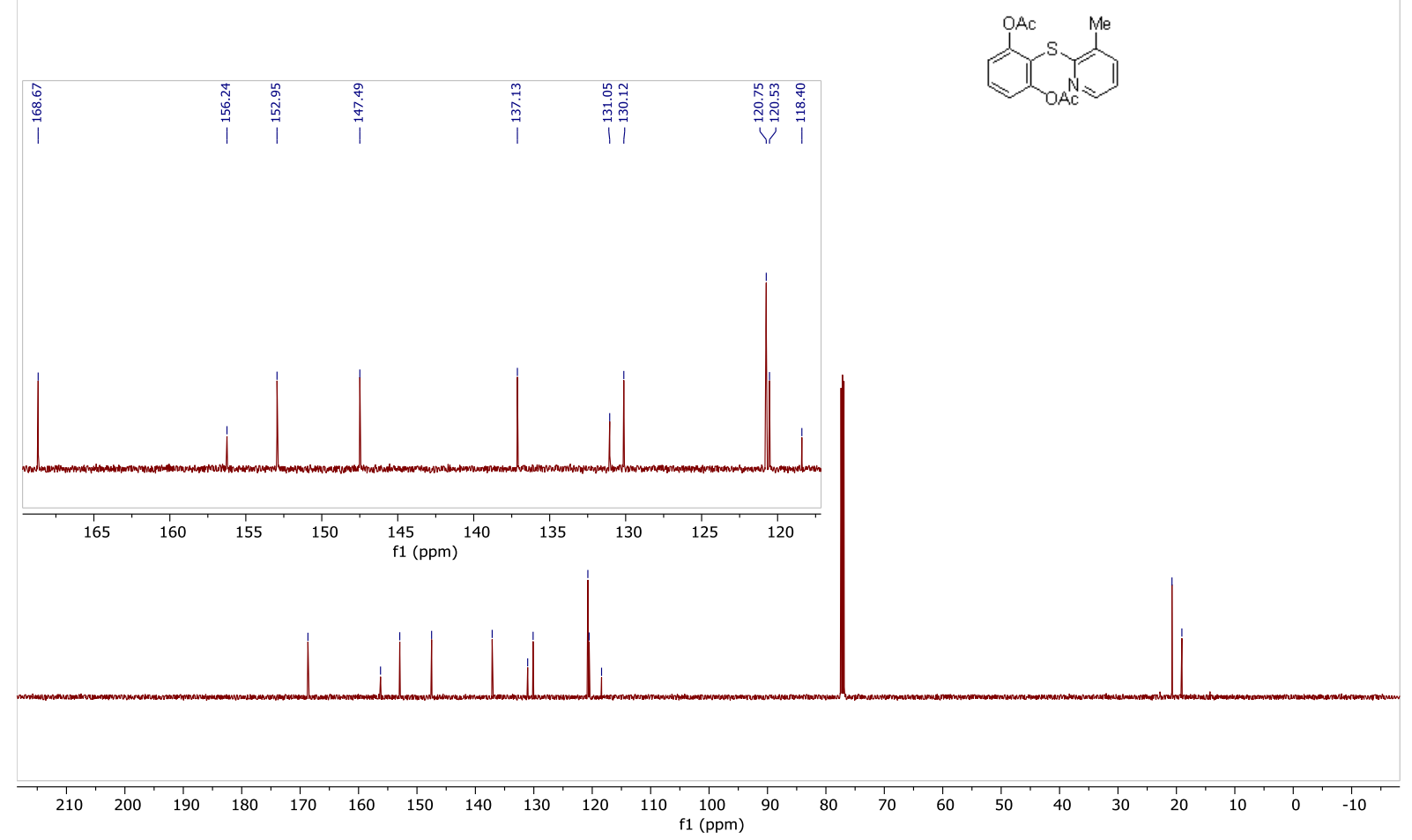


Figure S5.32: 2-(phenylsulfanyl)-3-bromo-5-methyl-pyridine (18h):
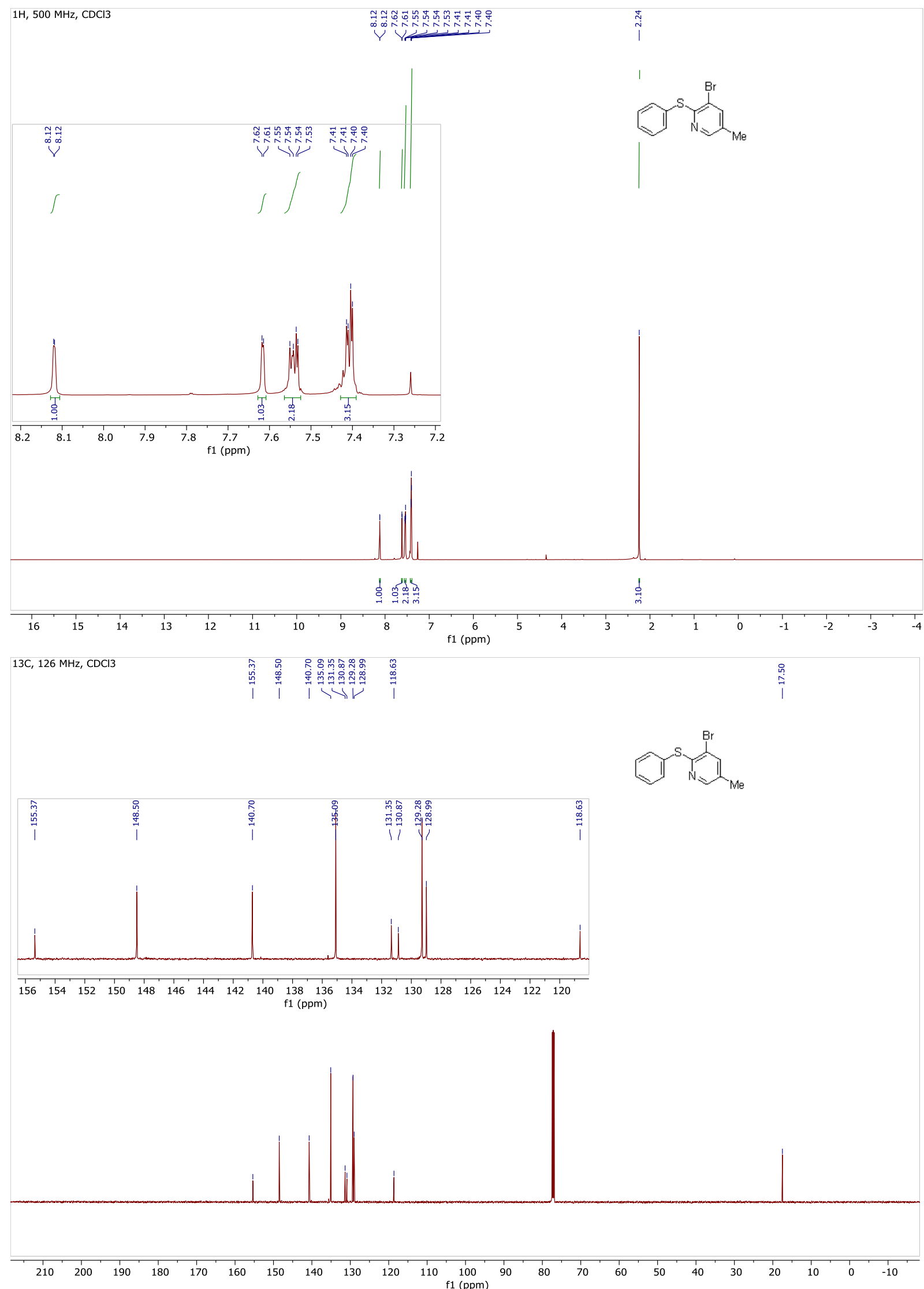
Figure S5.33: 2-(phenylsulfanyl)-3,5-dibromo-pyridine (14i):

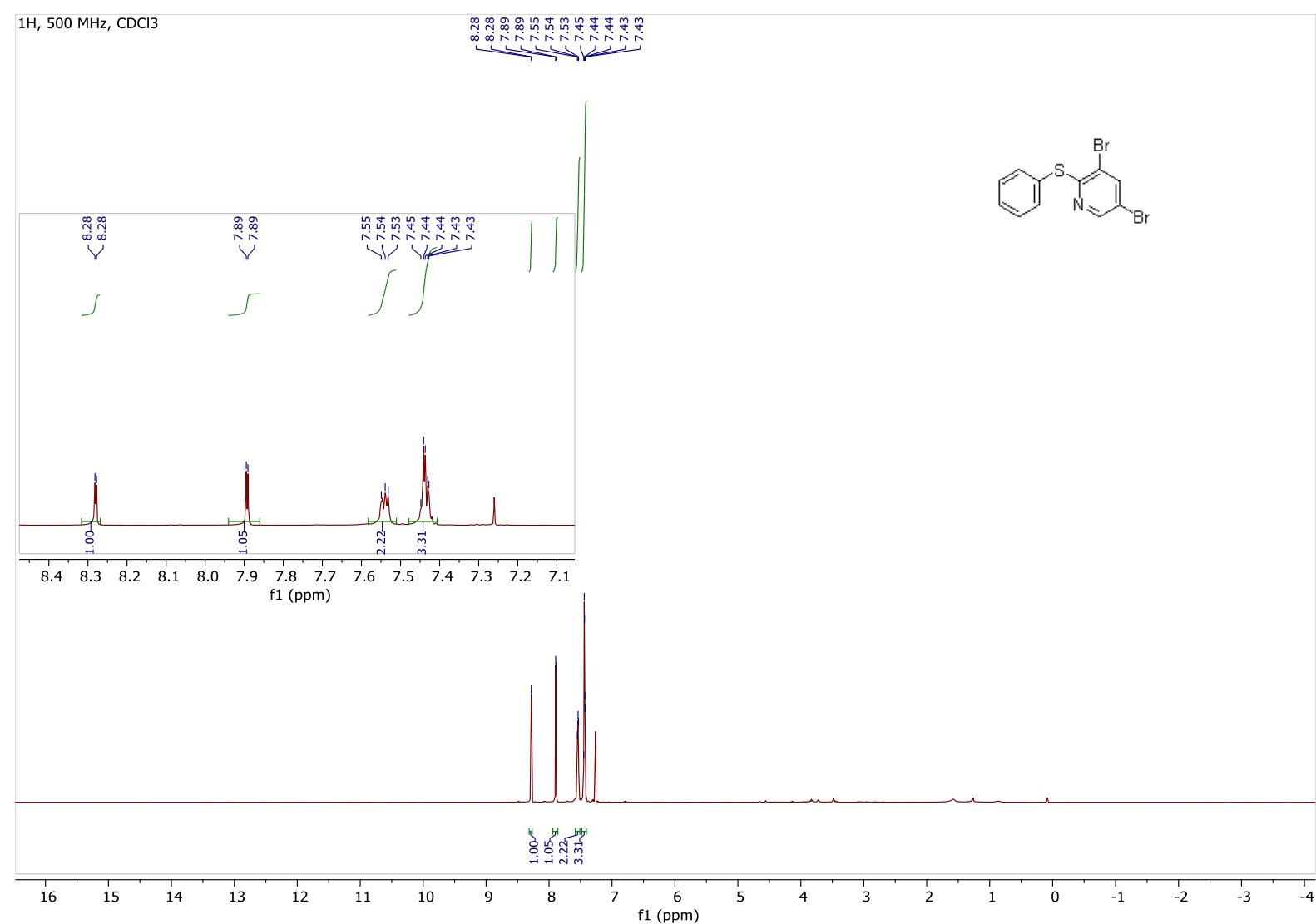

$13 \mathrm{C}, 126 \mathrm{MHz}, \mathrm{CDCl} 3$ 年
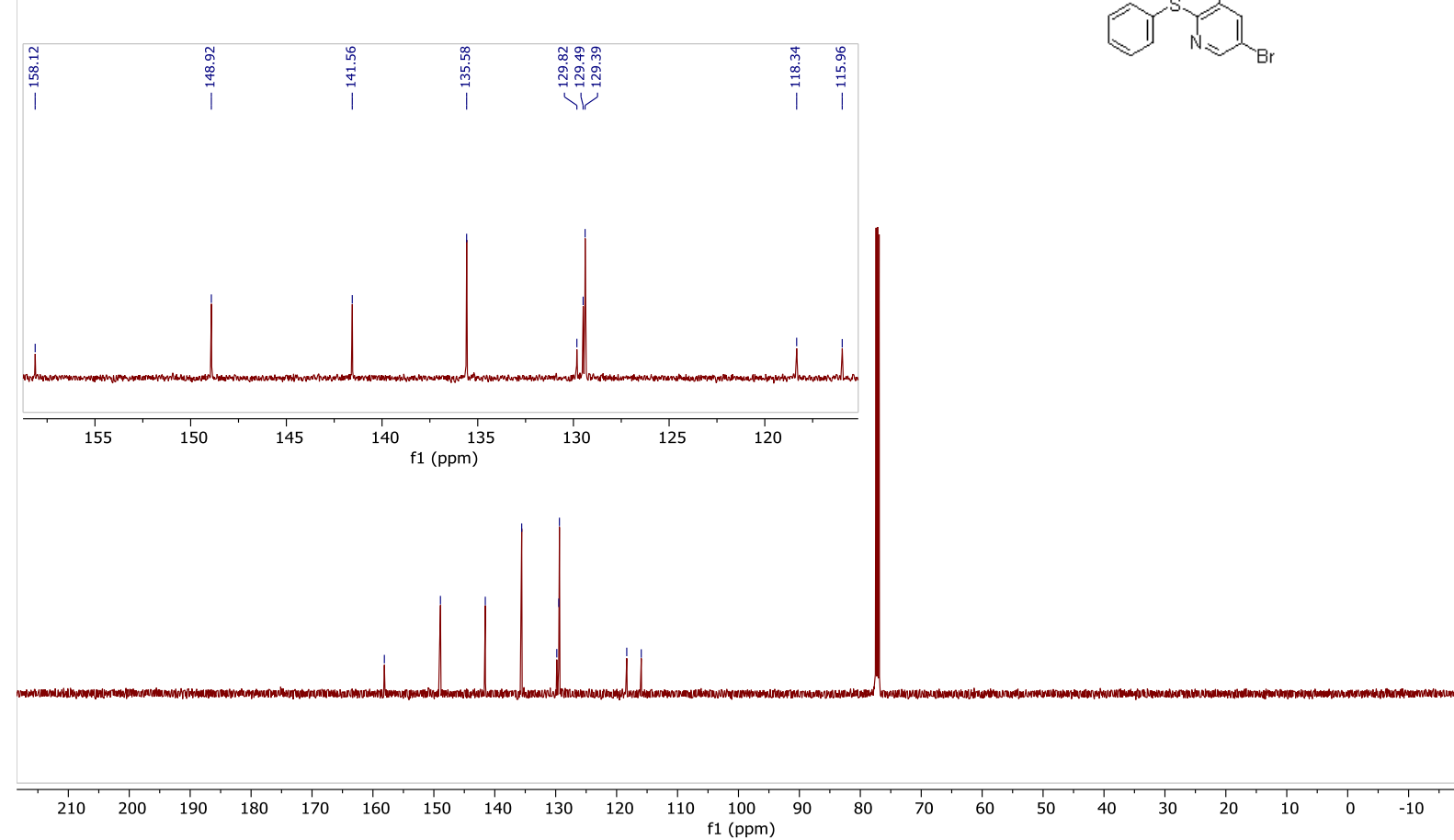
Figure S5.34: 2-(2,6-diphenyl-4-tert-butyl-phenyl)sulfanyl)-3-methyl-pyridine (19):
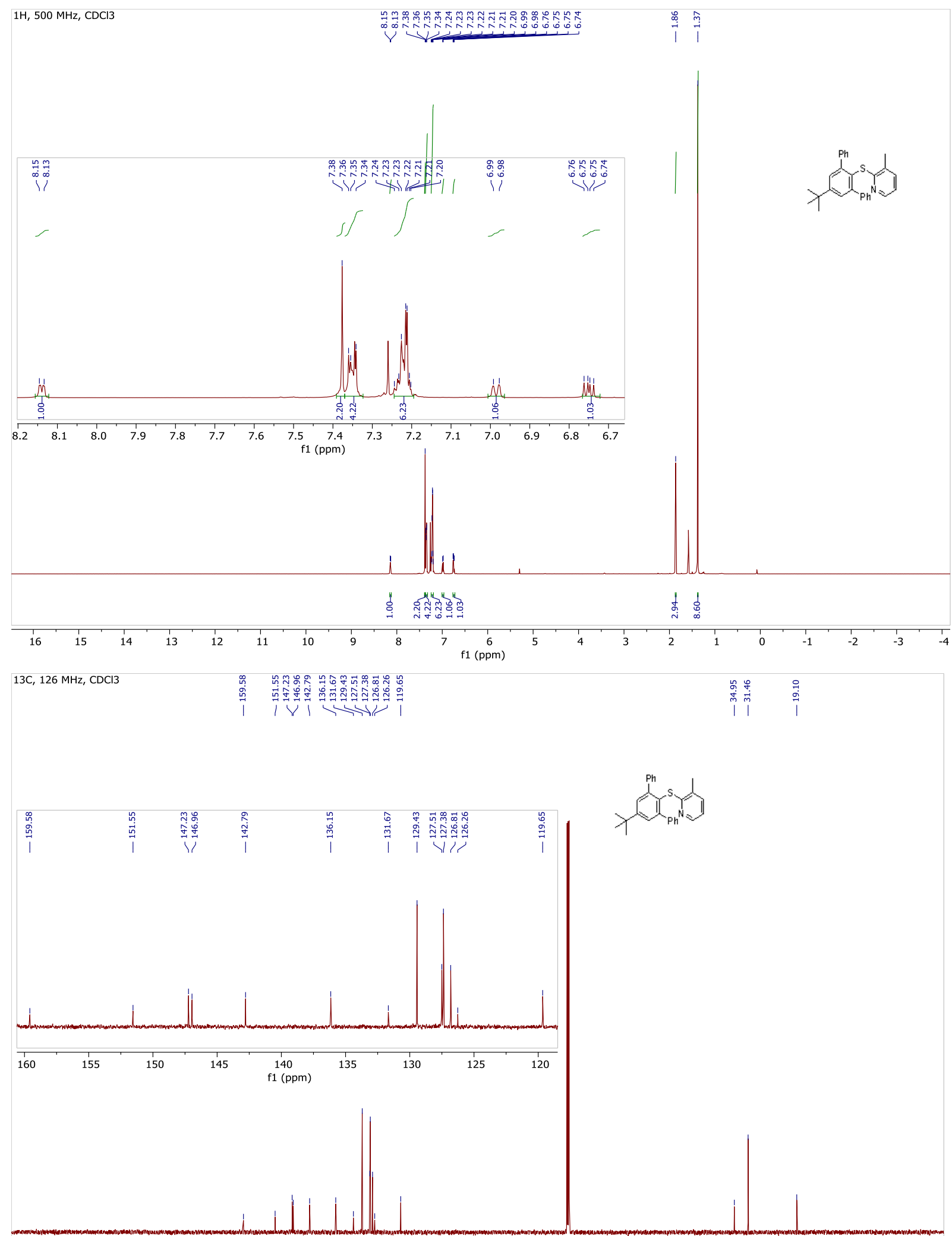

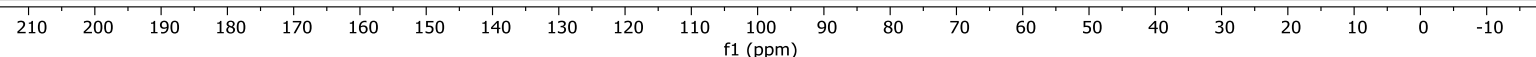


Figure S5.35: 2-(2,6-diphenyl-benzenesulfanyl)-3-methyl-pyridine (20):
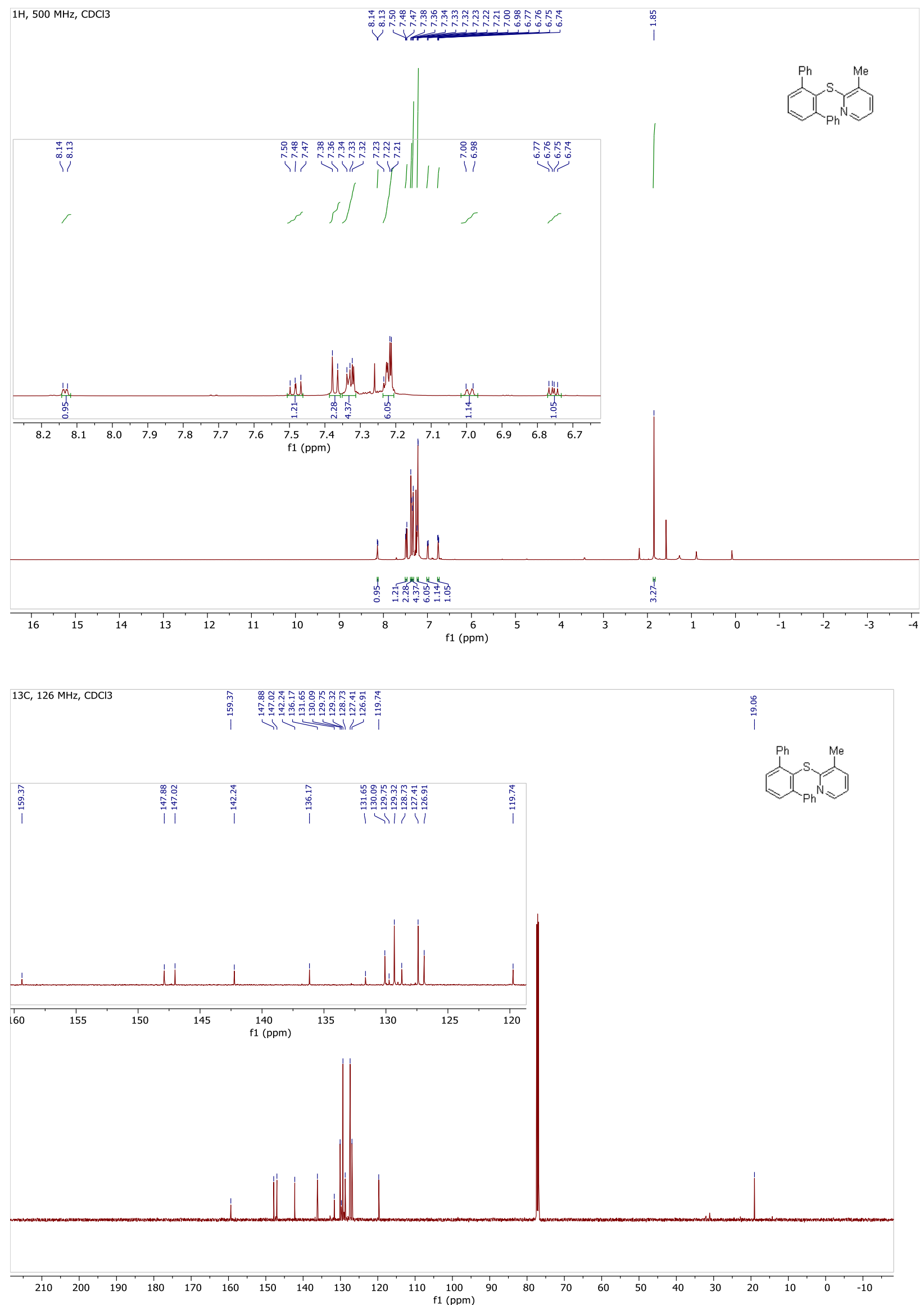
Figure S5.36: 2-(2,6-diphenyl-benzenesulfanyl)pyridine (21):

$1 \mathrm{H}, 500 \mathrm{MHz}, \mathrm{CDCl} 3$

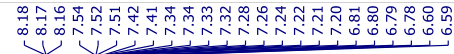
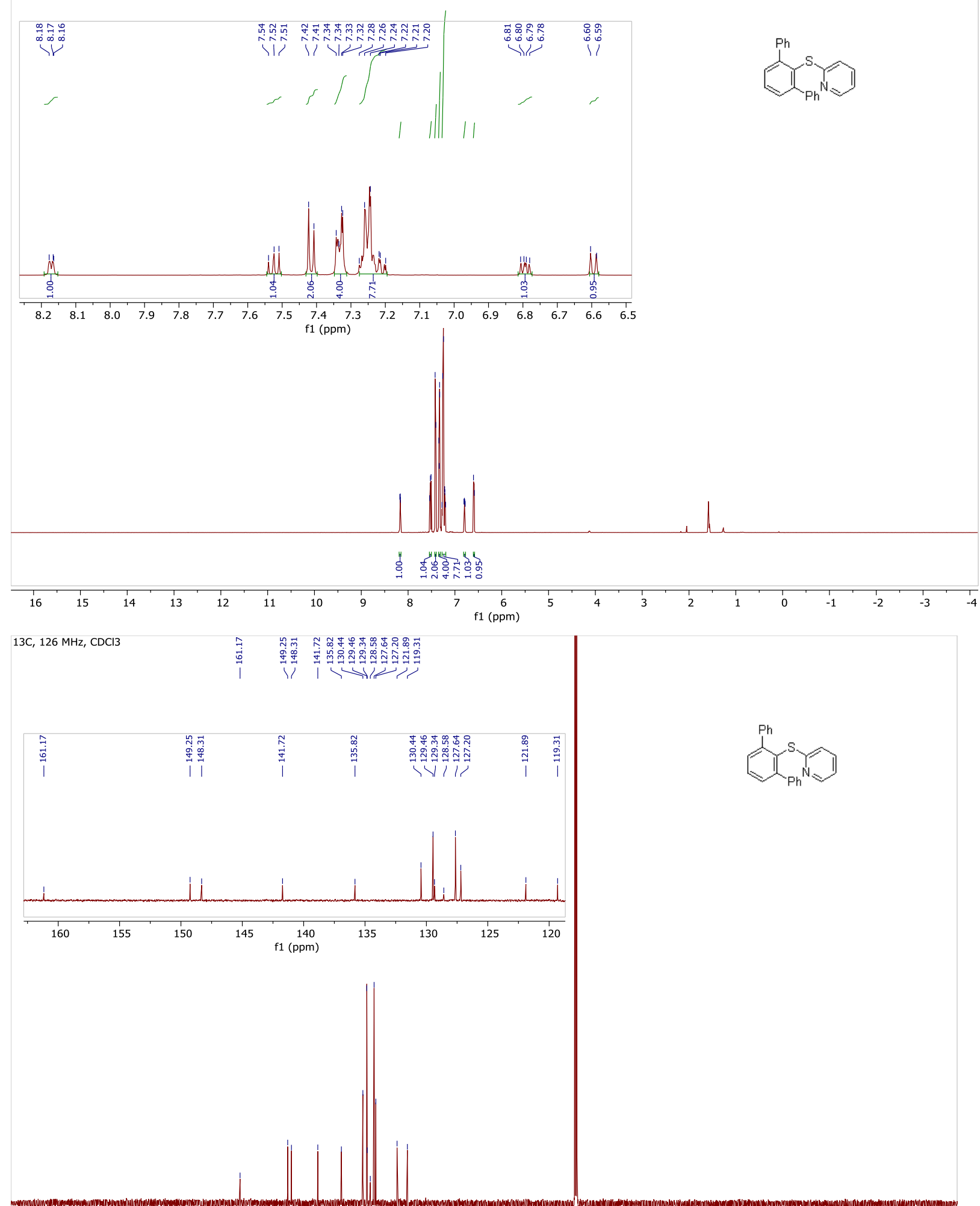

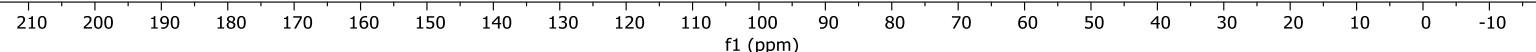


Figure S5.37: 2-(2-phenyl-6-chloro-sulfanyl)-3-methyl-pyridine (22):
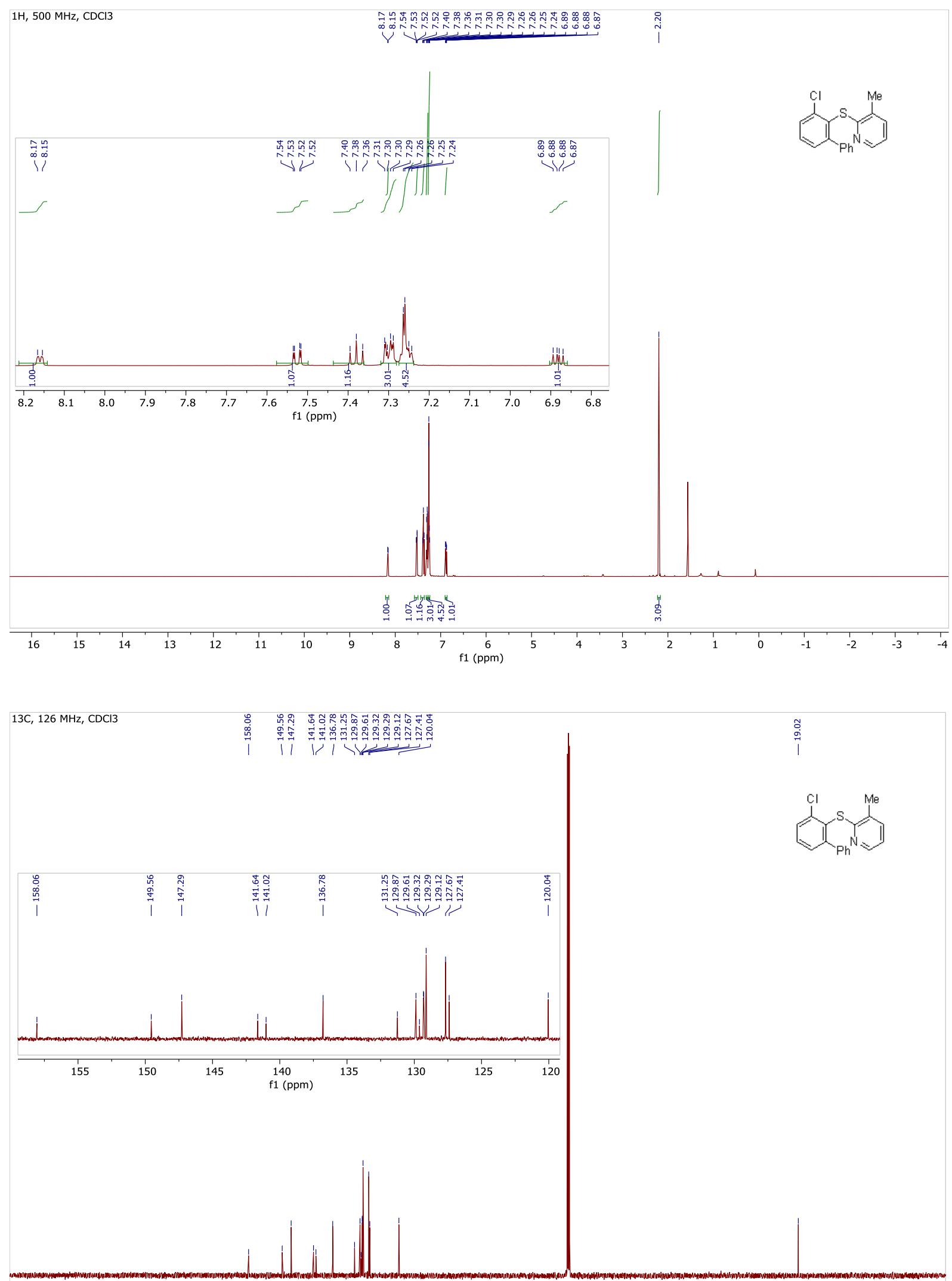

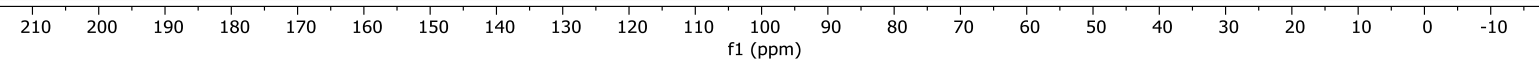


Figure S5.38: 2-(2-para-trifluoromethylbenzene-6-chloro-sulfanyl)-3-methyl-pyridine (23):

$1 \mathrm{H}, 500 \mathrm{MHz}, \mathrm{CDCl} 3$
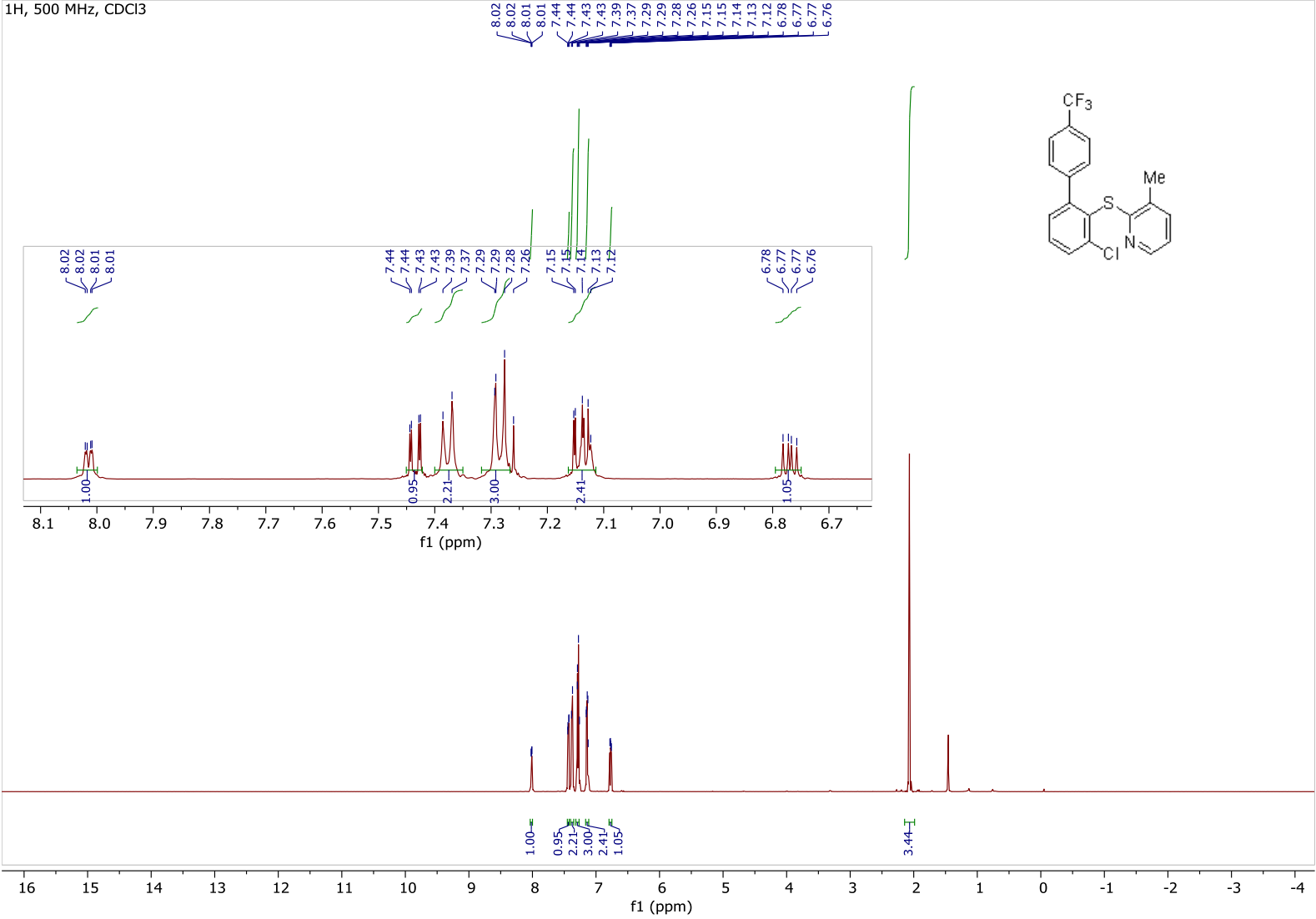

$19 \mathrm{~F}, 470 \mathrm{MHz}, \mathrm{CDCl} 3$
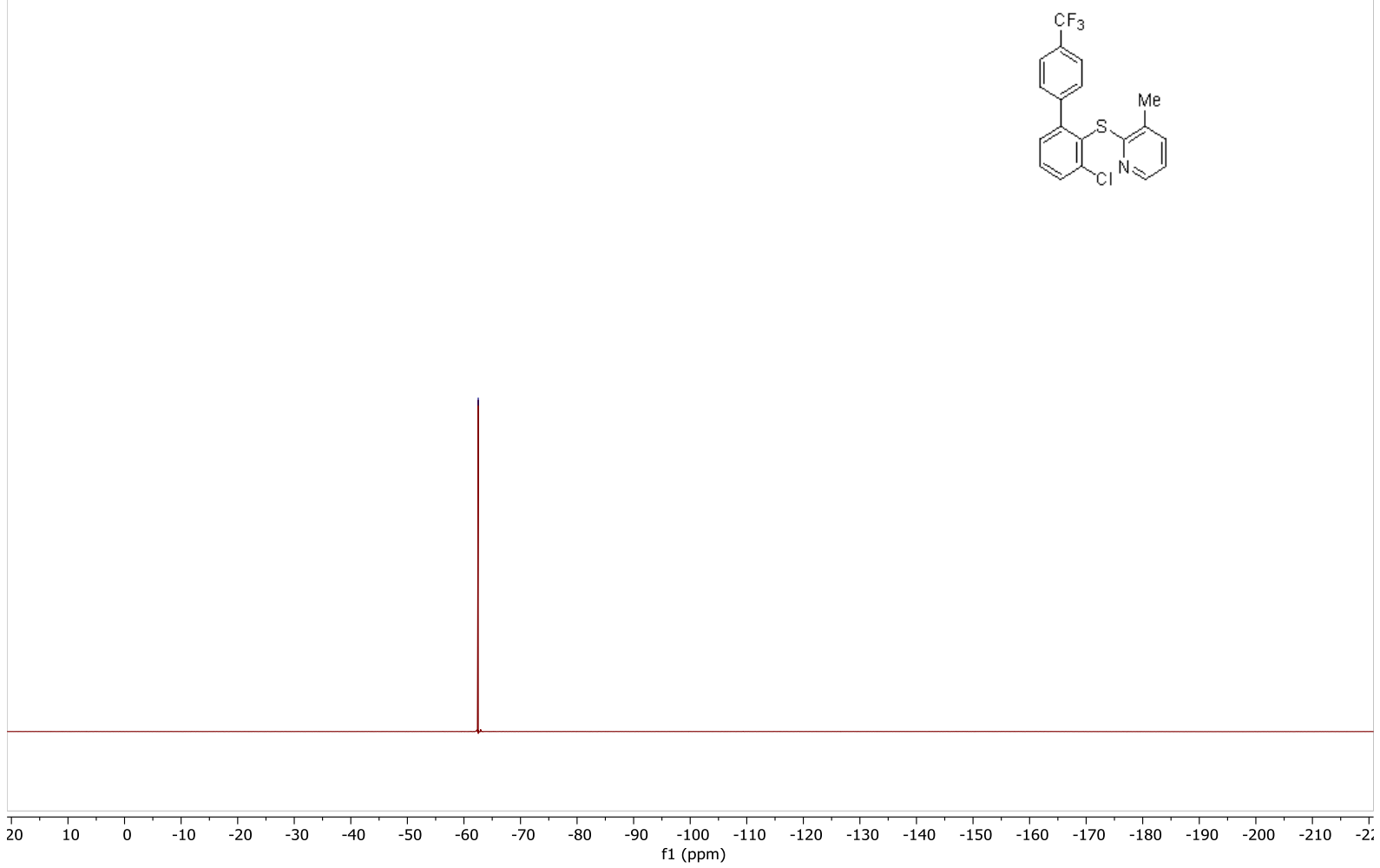

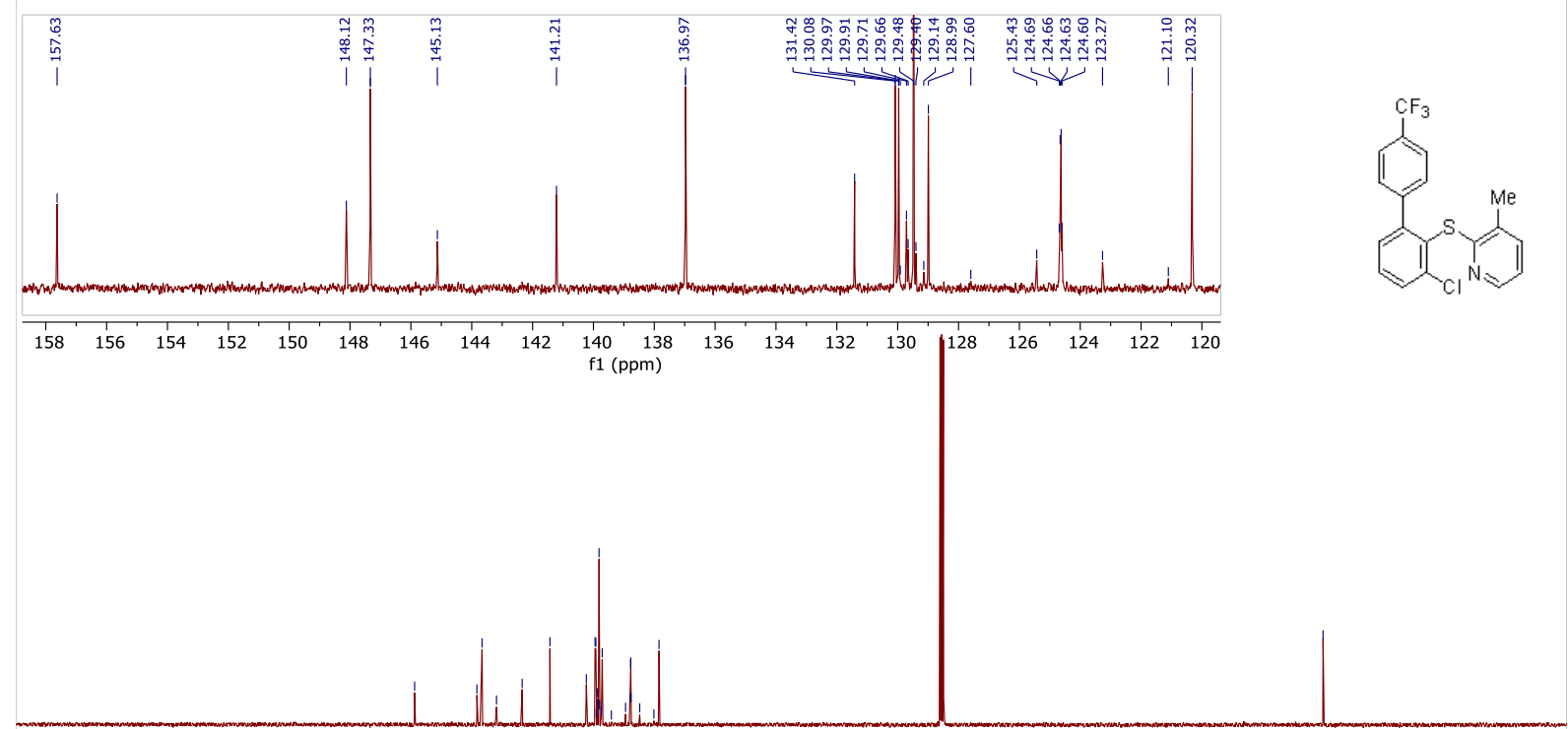

$\begin{array}{llllllllllllllllllllllll}\mid & 210 & 200 & 190 & 180 & 170 & 160 & 150 & 140 & 130 & 120 & 110 & 100 & 90 & 80 & 70 & 60 & 50 & 40 & 30 & 20 & 10 & 0 & -10\end{array}$ 
Figure S5.39: 2-(2-paramethoxybenzene-6-chloro-sulfanyl)-3-methyl-pyridine (24):

$1 \mathrm{H}, 500 \mathrm{MHz}, \mathrm{CDCl} 3$
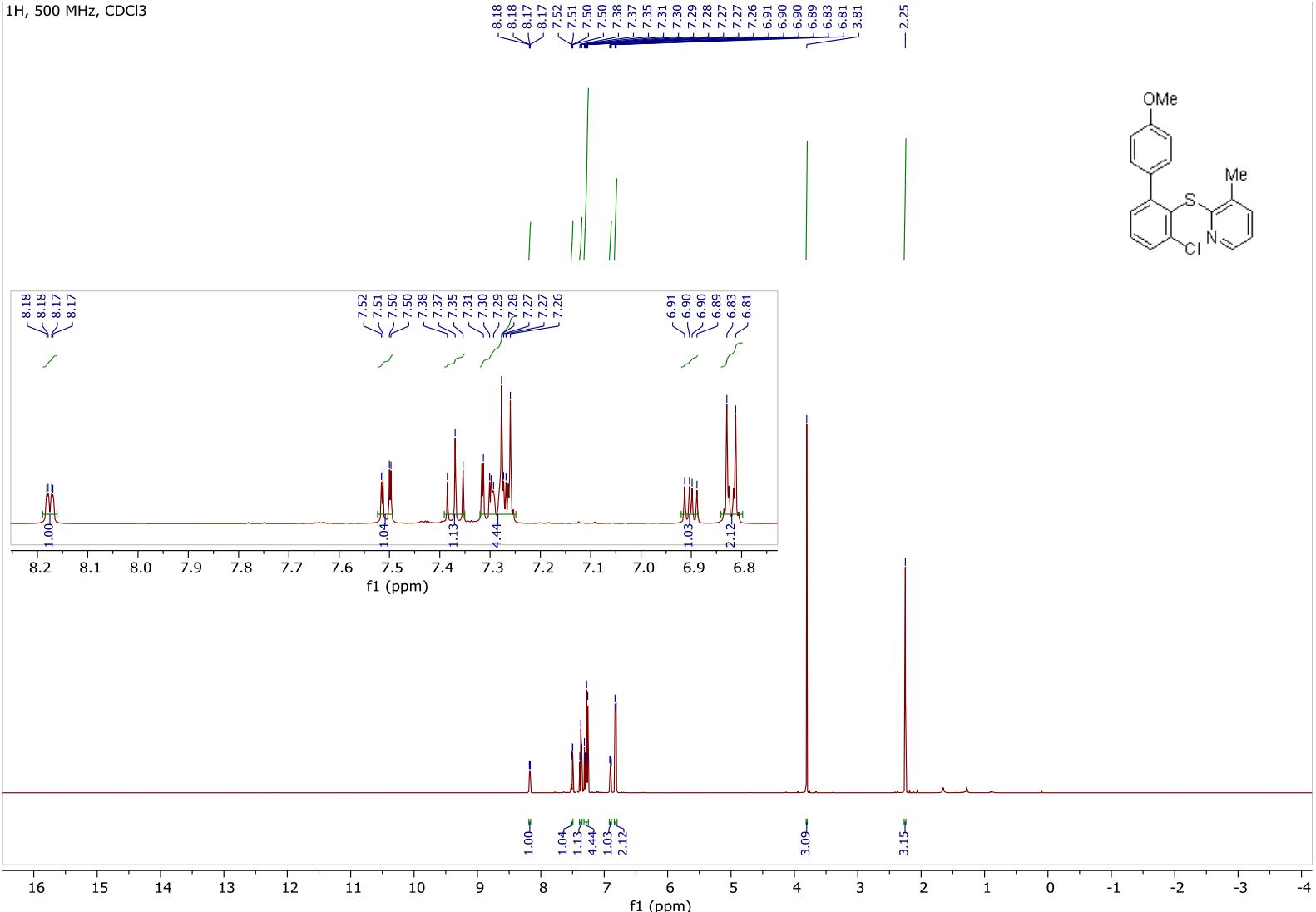

13C, $126 \mathrm{MHz}, \mathrm{CDCl} 3$
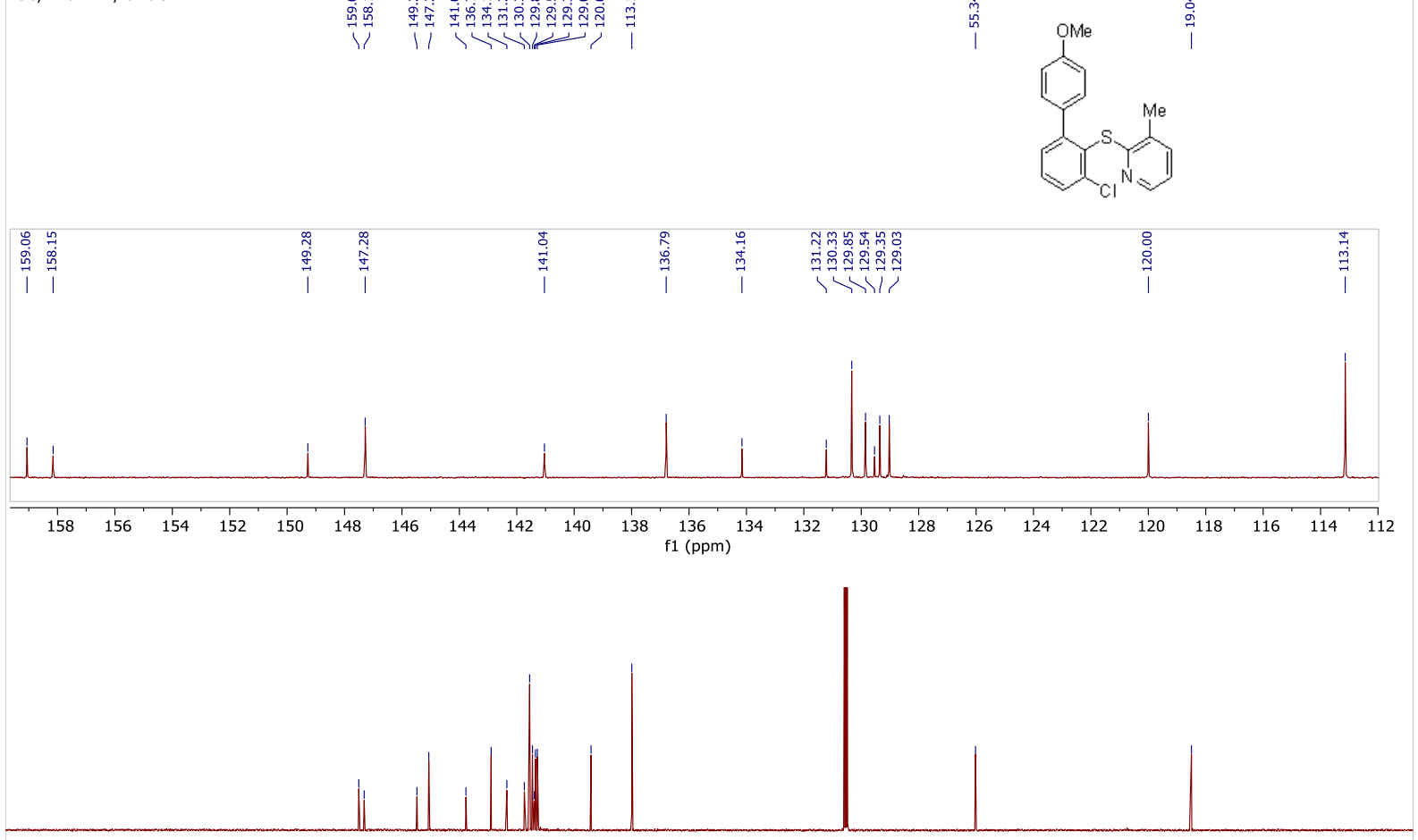

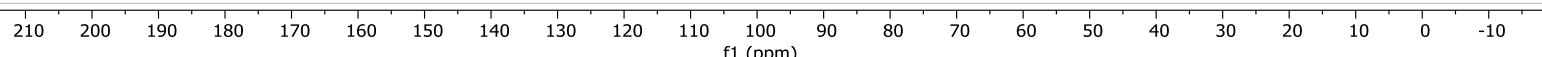




\section{REFERENCES}

(1) https://www.theses.fr/2018UBFCK047.

(2) Guilbaud, J.; Labonde, M.; A Selmi, A.; Kammoun, M.; Cattey, H.; Pirio, N.; Roger, J.; Hierso, J.-C. Palladium-Catalyzed Heteroaryl Thioethers Synthesis Overcoming Palladium Dithiolate Resting States Inertness: Practical Road to Sulfones and N-H-Sulfoximines. Catal. Com. 2018, 111, 52.

(3) Zhao, J.-L.; Chen, X. X.; Xie, H.; Ren, J.-T.; Gou, X.-F.; Sun, M. Palladium-Catalyzed Highly Regioselective ortho-Halogenation of 2-Pyridyl Sulfoxides. Synlett. 2017, 28, 1232, see the reported NMR data: ${ }^{1} \mathrm{H}$ NMR $\left(400 \mathrm{MHz}, \mathrm{CDCl}_{3}\right) \delta: 8.59(\mathrm{~d}, J=4.0 \mathrm{~Hz}, 1 \mathrm{H}), 7.94(\mathrm{~d}, J=8.0 \mathrm{~Hz}, 1 \mathrm{H}), 7.89-7.84$ $(\mathrm{m}, 2 \mathrm{H}), 7.56(\mathrm{dd}, J=0.8,4.0 \mathrm{~Hz}, 1 \mathrm{H}), 7.51-7.47(\mathrm{~m}, 1 \mathrm{H}), 7.36-7.20(\mathrm{~m}, 2 \mathrm{H})$.

(4) Testa, C.; Roger, J.; Scheib, S.; Fleurat-Lessard, P.; Hierso, J.-C. Palladium-Catalysed C-H Bond Electrophilic Fluorination of Highly Substituted Arylpyrazoles: Experimental and DFT Mechanistic Insights. Adv. Synth. Catal. 2015, 357, 2913.

(5) Guilbaud, J.; Labonde, M.; Cattey, H.; Contal, S.; Montalbetti, C.; Pirio, N.; Roger, J.; Hierso, J.-C. (2-Pyridyl)sulfonyl Groups for ortho-Directing Palladium-Catalyzed Carbon-Halogen Bond Formation at Functionalized Arenes. Adv. Synth. Catal. 2017, 21, 3792. 